\title{
Quantum Griffiths inequalities
}

\author{
Tadahiro Miyao \\ Department of Mathematics, Hokkaido University, \\ Sapporo 060-0810, Japan \\ E-mail: miyao@math.sci.hokudai.ac.jp
}

\begin{abstract}
We present a general framework of Griffiths inequalities for quantum systems. Our approach is based on operator inequalities associated with self-dual cones and provides a consistent viewpoint of the Griffiths inequality. As examples, we discuss the quantum Ising model, quantum rotor model, Bose-Hubbard model, and Hubbard model. We present a model-independent structure that governs the correlation inequalities.
\end{abstract}

\section{Introduction}

Ever since its formulation by Lenz [29], the Ising model has been the most fundamental model to illustrate the phenomenon of phase transitions. Let $\Lambda$ be a finite subset of $\mathbb{Z}^{d}$. The system's Hamiltonian is given by the function

$$
H_{\Lambda}(\sigma)=-\sum_{x, y \in \Lambda} J_{x y} \sigma_{x} \sigma_{y}
$$

for each $\sigma=\left\{\sigma_{x}\right\}_{x \in \Lambda} \in\{-1,+1\}^{\Lambda} . \quad J_{x y}$ is a non-negative coupling constant. The expectation value of the function $f:\{-1,+1\}^{\Lambda} \rightarrow \mathbb{R}$ is

$$
\langle f\rangle_{\beta}=\sum_{\sigma \in\{-1,+1\}^{\Lambda}} f(\sigma) \mathrm{e}^{-\beta H_{\Lambda}(\sigma)} / Z_{\beta},
$$

where $Z_{\beta}$ is the normalization constant $Z_{\beta}=\sum_{\sigma \in\{-1,+1\}^{\Lambda}} \mathrm{e}^{-\beta H_{\Lambda}(\sigma)}$. In [23], Griffiths discovered the following famous inequalities 1 :

- First Griffiths inequality:

$$
\left\langle\sigma_{A}\right\rangle_{\beta} \geq 0
$$

for each $A \subseteq \Lambda$, where $\sigma_{A}=\prod_{x \in A} \sigma_{x}$.

- Second Griffiths inequality:

$$
\left\langle\sigma_{A} \sigma_{B}\right\rangle_{\beta} \geq\left\langle\sigma_{A}\right\rangle_{\beta}\left\langle\sigma_{B}\right\rangle_{\beta}
$$

for each $A, B \subseteq \Lambda$.

\footnotetext{
${ }^{1}$ To be precise, this general formulation was established by Kelly and Sherman [32.
} 
Since Griffiths' discovery, a large number of rigorous studies on the Ising ferromagnets has been successfully undertaken by applying his inequalities. The fact that Griffiths inequalities are so useful indicates that they express the essence of correlations in the Ising system. Therefore, it is natural to ask whether similar inequalities hold true for other models. Studying this problem means trying to seek a model-independent or universal property of the notion of correlations. Griffiths inequalities already hold true for some classical models, e.g., the plane rotor model. This suggests that our problem is certainly meaningful. Ginibre took the first important step toward providing a general framework for Griffiths inequalities [20]. However, we know of only a few concrete examples of quantum (i.e., noncommutative) models that satisfy Griffiths inequalities [8, 10, 19, 36, 52]. Our goals here are as follows:

(a) To present a general method for constructing Griffiths inequalities for classical and quantum systems.

(b) According to (a), to highlight a universal property of correlations.

To this end, we advance the technique of operator inequalities associated with self-dual cones.

We already know that the quantum Ising and rotor models satisfy Griffiths inequalities. Thus, these two models can be regarded as role models for our purpose. A standard approach to proving the Griffiths inequality for these systems is to reduce the $d$-dimensional quantum systems to the corresponding $d+1$-dimensional classical systems using the Trotter-Kato product formula [4, 9, 10, 36]. However, since known proofs of the quantum Griffiths inequalities rely on the results of classical systems, it is difficult to extend these proofs to quantum models that cannot be reduced to classical ones. Considering this situation, we take the following steps:

(i) We prove the Griffiths inequality for the quantum Ising and rotor models using a method of operator inequalities and understand common mathematical structures underlying both models.

(ii) We seek similar structures in other models from our viewpoint of operator inequalities and construct the Griffiths inequality by analogy.

By carrying out these steps, we construct quantum Griffiths inequalities for the BoseHubbard and Hubbard models. We note that the proposed method can be applied to many other models, e.g., the Su-Schrieffer-Heeger (SSH) model, Holstein-Hubbard model, and Fröhlich mode2. Although we present a few concrete applications of our results here, we expect these inequalities to play important roles in statistical physics just as the original Griffiths inequalities did for the Ising system. We also remark that some of results in this paper can be proved by probabilisitc approaches, e.g., random walk representations. However, we believe that the proposed method can be applicable to a wider class of quantum models and clarify new aspects of the quantum Griffiths inequality. Finally, we emphasize the following: from the viewpoint of operator inequalities, we can find a common mathematical structure from among the several models mentioned above. This universal structure enables us to construct the Griffiths inequality for each model. From this fact, we expect to obtain a model-independent or general

\footnotetext{
${ }^{2}$ The problem of the quantum Heisenberg model is still open.
} 
expression of the notion of correlation from our viewpoint, see Section 8 for details.

This paper is organized as follows. In Section 2, we introduce a useful operator inequality induced by self-dual cones. Using this, we develop a general theory of the Griffiths inequality for quantum systems. In the following sections, we will demonstrate how our operator inequalities are effective for the study of correlation functions for quantum models.

In Section 3, we reformulate reflection positivity from the viewpoint of our operator inequalities. We then describe how we construct the Griffiths inequality using reflection positivity. This construction and the one in Section 2 are complementary to each other.

In Sections 4 and 5, we discuss the quantum Ising and rotor models, respectively. These sections provide not only something of a warm-up but also important clues for finding a common structure underlying the Griffiths inequality. Readers can learn how to use the operator inequalities through these sections as well.

Sections 6 and 7 are devoted to advanced applications of the abstract theory established in Sections 2 and 3. We construct the Griffiths inequality for the Bose-Hubbard model (Section 6), and Hubbard model (Section7). We emphasize that our constructions are natural modifications and extensions of the methods discussed in Sections 4 and 5 ,

In Section 8, we present concluding remarks. In Appendix A, we collect useful propositions concerning our operator inequalities. These propositions will be used repeatedly in this study.

Acknowledgements: This work was supported by KAKENHI(20554421). I would be grateful to the anonymous referee for useful comments.

\section{General theory}

\section{$2.1 \quad$ First inequality}

Let $(\mathfrak{H},\langle\cdot \mid \cdot\rangle)$ be a complex Hilbert space and $\mathfrak{P}$ be a convex cone in $\mathfrak{H}$. The dual cone $\mathfrak{P}^{\dagger}$ of $\mathfrak{P}$ is defined as

$$
\mathfrak{P}^{\dagger}=\{x \in \mathfrak{H} \mid\langle x \mid y\rangle \geq 0 \forall y \in \mathfrak{P}\} .
$$

We say that $\mathfrak{P}$ is self-dual if

$$
\mathfrak{P}=\mathfrak{P}^{\dagger} \text {. }
$$

Henceforth, we always assume that $\mathfrak{P}$ is self-dual. Each element $x$ in $\mathfrak{P}$ is called positive w.r.t. $\mathfrak{P}$ and written as $x \geq 0$ w.r.t. $\mathfrak{P}$.

Definition 2.1 Let $\mathscr{B}(\mathfrak{H})$ be the set of all bounded linear operators on $\mathfrak{H}$. Let $A \in$ $\mathscr{B}(\mathfrak{H})$. If $A x \geq 0$ w.r.t. $\mathfrak{P}$ for all $x \in \mathfrak{P}$, then we say that $A$ preserves the positivity w.r.t. $\mathfrak{P}$ and write 3

$$
A \unrhd 0 \quad \text { w.r.t. } \mathfrak{P} .
$$

\footnotetext{
${ }^{3}$ This symbol was introduced by Miura 42 . Bratteli, Kishimoto and Robinson studied the commutative cases in [7, 35].
} 
Note that

$$
A \unrhd 0 \text { w.r.t. } \mathfrak{P} \Longrightarrow\langle x \mid A y\rangle \geq 0 \quad \forall x, y \in \mathfrak{P} .
$$

The following proposition is often useful.

Proposition 2.2 [42] We have the following:

(i) If $A \unrhd 0, B \unrhd 0$ w.r.t. $\mathfrak{P}$ and $\alpha \geq 0, \beta \geq 0$, then $\alpha A+\beta B \unrhd 0$ w.r.t. $\mathfrak{P}$.

(ii) If $A \unrhd 0$ and $B \unrhd 0$ w.r.t. $\mathfrak{P}$, then $A B \unrhd 0$ w.r.t. $\mathfrak{P}$.

Our first setting is as follows.

(A) There exists a complete orthonormal system (CONS) $\left\{e_{n}\right\}_{n \in \mathbb{N}}$ of $\mathfrak{H}$ such that $e_{n} \in \mathfrak{P}$ for all $n \in \mathbb{N}$.

The system's Hamiltonian is denoted by $H . H$ is self-adjoint and bounded from below. To state the first quantum (i.e., noncommutative) Griffiths inequality, we need the following conditions:

(H. 1) $\mathrm{e}^{-\beta H} \unrhd 0$ w.r.t. $\mathfrak{P}$ for all $\beta \geq 0$.

For each $A \in \mathscr{B}(\mathfrak{H})$, the thermal expectation value of $A$ is defined as

$$
\langle A\rangle_{\beta}=\operatorname{Tr}\left[A \mathrm{e}^{-\beta H}\right] / Z_{\beta}, \quad Z_{\beta}=\operatorname{Tr}\left[\mathrm{e}^{-\beta H}\right] .
$$

Remark 2.3 In this section, we always assume that $\mathrm{e}^{-\beta H}$ is in the trace class for all $\beta>0 . \diamond$

Theorem 2.4 is a prototype of the Griffiths inequality.

Theorem 2.4 Assume (A) and (H. 1). If $A \unrhd 0$ w.r.t. $\mathfrak{P}$, then $\langle A\rangle_{\beta} \geq 0$ for all $\beta \geq 0$.

Proof. By our assumptions and Proposition 2.2 , we have $A \mathrm{e}^{-\beta H} \unrhd 0$ w.r.t. $\mathfrak{P}$ for all $\beta \geq 0$. Thus, applying Proposition A.1, we conclude Theorem 2.4,

To discuss the case where $\beta=\infty$, we assume that

(A') $H$ has a unique ground state, i.e., $\operatorname{dim} \operatorname{ker}(H-E)=1$, where $E=\inf \operatorname{spec}(H)$.

Under this condition, we can define the ground state expectation value as

$$
\langle A\rangle_{\infty}=\langle\psi \mid A \psi\rangle, \quad A \in \mathscr{B}(\mathfrak{H}),
$$

where $\psi$ is the unique ground state of $H$ such that $\|\psi\|=1$.

Theorem 2.5 Assume (A') and (H. 1). If $A \unrhd 0$ w.r.t. $\mathfrak{P}$, then $\langle A\rangle_{\infty} \geq 0$. 
Proof. By Proposition A.6, we can choose $\psi$ as $\psi \geq 0$ w.r.t. $\mathfrak{P}$. Thus, this theorem immediately follows from (2.3).

Remark 2.6 If we assume that $\mathrm{e}^{-\beta H}$ improves the positivity w.r.t. $\mathfrak{P}$ for all $\beta>0$, then the ground state of $H$ is automatically unique, see 8.4 for details. $\diamond$

Theorem 2.7 is a generalization of Theorem 2.4 ,

Theorem 2.7 Assume (A) and (H. 1). Let $A(s)=\mathrm{e}^{-s H} A \mathrm{e}^{s H}$. If $A_{j} \unrhd 0$ w.r.t. $\mathfrak{P}$ for all $j=1, \ldots, n$, we then have

$$
\left\langle\prod_{j=1}^{n} A_{j}\left(s_{j}\right)\right\rangle_{\beta} \geq 0
$$

for all $0 \leq s_{1} \leq s_{2} \leq \cdots \leq s_{n}<\beta$, where $\prod_{j=1}^{\stackrel{n}{\rightarrow}} O_{j}=O_{1} O_{2} \cdots O_{n}$, the ordered product.

Proof. Let $\mathcal{S}=\left[\prod_{j=1}^{n} A_{j}\left(s_{j}\right)\right] \mathrm{e}^{-\beta H}$. By our assumptions, we see that

$$
\mathcal{S}=\underbrace{\mathrm{e}^{-s_{1} H}}_{\unrhd 0} \underbrace{A_{1}}_{\unrhd 0} \underbrace{\mathrm{e}^{-\left(s_{2}-s_{1}\right) H}}_{\unrhd 0} \cdots \underbrace{A_{n}}_{\unrhd 0} \underbrace{\mathrm{e}^{-\left(\beta-s_{n}\right) H}}_{\unrhd 0} \unrhd 0 \quad \text { w.r.t. } \mathfrak{P} .
$$

Thus, by Proposition A.1, we obtain (2.6).

Theorem 2.8 Assume (A') and (H. 1). Then (2.6) holds true at $\beta=\infty$.

\subsection{Second inequality}

We consider the extended Hilbert space $\mathfrak{H}_{\text {ext }}=\mathfrak{H} \otimes \mathfrak{H}$. Let $\mathfrak{P}_{\text {ext }}$ be a self-dual cone in $\mathfrak{H} \otimes \mathfrak{H}$. Instead of $(\mathbf{A})$, we assume the following:

(B) There exists a CONS $\left\{E_{n}\right\}_{n \in \mathbb{N}}$ of $\mathfrak{H}_{\text {ext }}$ such that $E_{n} \in \mathfrak{P}_{\text {ext }}$ for all $n \in \mathbb{N}$.

To state Theorem 2.9, the following condition is assumed:

(H. 2) Let $H_{\text {ext }}=H \otimes \mathbb{1}+\mathbb{1} \otimes H$. Then there exists a unitary operator $\mathscr{U}$ such that

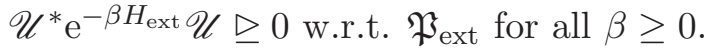

There are several ways to state the second quantum Griffiths inequality. First, we give the following formulation.

Theorem 2.9 Assume (B) and (H. 2). Let $A, B, C, D \in \mathscr{B}(\mathfrak{H})$ and $A(s)=\mathrm{e}^{-s H} A \mathrm{e}^{s H}$. Assume the following:

(i) $\mathscr{U}^{*} A \otimes C \mathscr{U} \unrhd 0$ w.r.t. $\mathfrak{P}_{\text {ext }}$.

(ii) $\mathscr{U}^{*}(B \otimes D-D \otimes B) \mathscr{U} \unrhd 0$ w.r.t. $\mathfrak{P}_{\text {ext }}$. 
Then we have

$$
\langle A(s) B(t)\rangle_{\beta}\langle C(s) D(t)\rangle_{\beta}-\langle A(s) D(t)\rangle_{\beta}\langle C(s) B(t)\rangle_{\beta} \geq 0
$$

for all $0 \leq s \leq t<\beta$. In addition, assume (A) and (H. 1). If $A \unrhd 0, B \unrhd 0, C \unrhd 0$ and $D \unrhd 0$ w.r.t. $\mathfrak{P}$, we obtain

$$
\langle A(s) B(t)\rangle_{\beta} \geq 0, \quad\langle C(s) D(t)\rangle_{\beta} \geq 0, \quad\langle A(s) D(t)\rangle_{\beta} \geq 0, \quad\langle C(s) B(t)\rangle_{\beta} \geq 0
$$

for all $0 \leq s \leq t<\beta$.

Proof. Let

$$
\left\langle\langle X\rangle_{\beta}=\operatorname{Tr}\left[X \mathrm{e}^{-\beta H_{\mathrm{ext}}}\right] / Z_{\beta}^{2} .\right.
$$

Then we can derive (2.8) from the following:

$$
\langle\langle A(s) \otimes C(s)(B(t) \otimes D(t)-D(t) \otimes B(t))\rangle\rangle_{\beta} \geq 0 .
$$

But this follows immediately from Proposition A.1 and the fact that

$$
\begin{aligned}
& \mathscr{U}^{*} A(s) \otimes C(s)(B(t) \otimes D(t)-D(t) \otimes B(t)) \mathrm{e}^{-\beta H_{\text {ext }} \mathscr{U}} \\
= & \mathscr{U}^{*} \mathrm{e}^{-s H_{\text {ext }}} A \otimes C \mathrm{e}^{-(t-s) H_{\text {ext }}}(B \otimes D-D \otimes B) \mathrm{e}^{-(\beta-t) H_{\text {ext }}} \mathscr{U} \unrhd 0
\end{aligned}
$$

w.r.t. $\mathfrak{P}_{\text {ext }}$ for all $0 \leq s \leq t<\beta$.

By (H. 1), it follows that $\mathrm{e}^{-s H} A \mathrm{e}^{-(t-s) H} B \mathrm{e}^{-(\beta-t) H} \unrhd 0$ w.r.t. $\mathfrak{P}$ for all $\beta \geq 0$. Thus, by Proposition A.1, we obtain that $\langle A(s) B(t)\rangle_{\beta} \geq 0$ for all $\beta \geq 0$.

Theorem 2.10 If we replace (A) and (B) by (A') in Theorem [2.9, then (2.8) and (2.9) hold true at $\beta=\infty$.

Proof. Since $H$ has a unique ground state $\psi, H_{\text {ext }}$ has a unique ground state $\psi \otimes \psi$ as well. By (H. 2) and Proposition A.6, it follows that $\Phi=\mathscr{U}^{*} \psi \otimes \psi \geq 0$ w.r.t. $\mathfrak{P}_{\text {ext }}$. Thus, by (2.3),

$$
\begin{aligned}
& \left\langle\left\langle\mathrm{e}^{-s H_{\mathrm{ext}}} A \otimes C \mathrm{e}^{-(t-s) H_{\mathrm{ext}}}(B \otimes D-D \otimes B) \mathrm{e}^{t H_{\mathrm{ext}}}\right\rangle\right\rangle_{\infty} \\
= & \mathrm{e}^{2(t-s) E}\langle\underbrace{\Phi}_{\geq 0} \mid \underbrace{\mathscr{U}^{*} A \otimes C \mathrm{e}^{-(t-s) H_{\mathrm{ext}}}(B \otimes D-D \otimes B) \mathscr{U}}_{\unrhd 0} \underbrace{\Phi}_{\geq 0}\rangle \geq 0,
\end{aligned}
$$

where $\langle\langle X\rangle\rangle_{\infty}=\langle\psi \otimes \psi \mid X \psi \otimes \psi\rangle$. This completes the proof.

We introduce the Duhamel two-point function,

$$
(A, B)_{\beta}=Z_{\beta}^{-1} \int_{0}^{1} \operatorname{Tr}\left[A \mathrm{e}^{-x \beta H} B \mathrm{e}^{-(1-x) \beta H}\right] d x, \quad A, B \in \mathscr{B}(\mathfrak{H}) .
$$


Corollary 2.11 Assume (B) and (H. 2). Let $A, B \in \mathscr{B}(\mathfrak{H})$. Assume the following:

(i) $\mathscr{U}^{*} A \otimes \mathbb{1} \mathscr{U} \unrhd 0$ w.r.t. $\mathfrak{P}_{\mathrm{ext}}$.

(ii) $\mathscr{U}^{*}(B \otimes \mathbb{1}-\mathbb{1} \otimes B) \mathscr{U} \unrhd 0$ w.r.t. $\mathfrak{P}_{\mathrm{ext}}$.

Then we have

$$
\begin{aligned}
(A, B)_{\beta}-\langle A\rangle_{\beta}\langle B\rangle_{\beta} & \geq 0, \\
\langle A B\rangle_{\beta}-\langle A\rangle_{\beta}\langle B\rangle_{\beta} & \geq 0 .
\end{aligned}
$$

In addition, assume (A) and (H. 1). If $A \unrhd 0$ and $B \unrhd 0$ w.r.t. $\mathfrak{P}$, we obtain

$$
(A, B)_{\beta} \geq 0, \quad\langle A B\rangle_{\beta} \geq 0, \quad\langle A\rangle_{\beta} \geq 0, \quad\langle B\rangle_{\beta} \geq 0 .
$$

Our second formulation of the second quantum Griffiths inequality is as follows.

Theorem 2.12 Assume (B) and (H. 2). Let $A, B, C, D \in \mathscr{B}(\mathfrak{H})$ and $A(s)=\mathrm{e}^{-s H} A \mathrm{e}^{s H}$. Assume the following:

$$
\mathscr{U}^{*}(A \otimes C-C \otimes A) \mathscr{U} \unrhd 0, \quad \mathscr{U}^{*}(B \otimes D-D \otimes B) \mathscr{U} \unrhd 0 \quad \text { w.r.t. } \mathfrak{P}_{\text {ext }} .
$$

Then we have

$$
\langle A(s) B(t)\rangle_{\beta}\langle C(s) D(t)\rangle_{\beta}-\langle A(s) D(t)\rangle_{\beta}\langle C(s) B(t)\rangle_{\beta} \geq 0
$$

for all $0 \leq s \leq t<\beta$. In addition, assume (A) and (H. 1). If $A \unrhd 0, B \unrhd 0, C \unrhd 0$ and $D \unrhd 0$ w.r.t. $\mathfrak{P}$, we obtain

$$
\langle A(s) B(t)\rangle_{\beta} \geq 0, \quad\langle C(s) D(t)\rangle_{\beta} \geq 0, \quad\langle A(s) D(t)\rangle_{\beta} \geq 0, \quad\langle C(s) B(t)\rangle_{\beta} \geq 0
$$

for all $0 \leq s \leq t<\beta$.

Proof. Note that we can conclude (2.19) from the following:

$$
\langle\langle(A(s) \otimes C(s)-C(s) \otimes A(s))(B(t) \otimes D(t)-D(t) \otimes B(t))\rangle\rangle_{\beta} \geq 0 .
$$

To show this, we use Proposition A.1 and the fact that

$$
\begin{aligned}
& \mathscr{U}^{*}(A(s) \otimes C(s)-C(s) \otimes A(s))(B(t) \otimes D(t)-D(t) \otimes B(t)) \mathrm{e}^{-\beta H_{\text {ext }} \mathscr{U}} \\
= & \mathscr{U}^{*} \mathrm{e}^{-s H_{\text {ext }}}(A \otimes C-C \otimes A) \mathrm{e}^{-(t-s) H_{\text {ext }}}(B \otimes D-D \otimes B) \mathrm{e}^{-(\beta-t) H_{\text {ext }}} \mathscr{U} \unrhd 0
\end{aligned}
$$

w.r.t. $\mathfrak{P}_{\text {ext }}$ for all $0 \leq s \leq t<\beta$.

Theorem 2.13 If we replace $(\mathbf{A})$ and $(\mathbf{B})$ by $\left(\mathbf{A}^{\prime}\right)$ in Theorem 2.12, then (2.19) and (2.20) hold true at $\beta=\infty$. 
Corollary 2.14 Assume (B) and (H. 2). Let $A, B \in \mathscr{B}(\mathfrak{H})$. Assume the following:

$$
\mathscr{U}^{*}(A \otimes \mathbb{1}-\mathbb{1} \otimes A) \mathscr{U} \unrhd 0, \quad \mathscr{U}^{*}(B \otimes \mathbb{1}-\mathbb{1} \otimes B) \mathscr{U} \unrhd 0 \quad \text { w.r.t. } \mathfrak{P}_{\mathrm{ext}} .
$$

Then we have

$$
\langle A(s) B(t)\rangle_{\beta}-\langle A\rangle_{\beta}\langle B\rangle_{\beta} \geq 0
$$

for all $0 \leq s \leq t<\beta$. In particular, we have

$$
\begin{array}{r}
(A, B)_{\beta}-\langle A\rangle_{\beta}\langle B\rangle_{\beta} \geq 0, \\
\langle A B\rangle_{\beta}-\langle A\rangle_{\beta}\langle B\rangle_{\beta} \geq 0 .
\end{array}
$$

In addition, assume (A) and (H. 1). If $A \unrhd 0, B \unrhd 0$ w.r.t. $\mathfrak{P}$, then we obtain

$$
(A, B)_{\beta} \geq 0, \quad\langle A B\rangle_{\beta} \geq 0, \quad\langle A\rangle_{\beta} \geq 0, \quad\langle B\rangle_{\beta} \geq 0 .
$$

\subsection{Further generalization}

Theorem 2.12 can be generalized as follows.

Theorem 2.15 Assume (B) and (H. 2). Let $A_{j}, B_{j} \in \mathscr{B}(\mathfrak{H}), j=1, \ldots, n$ and $A(s)=\mathrm{e}^{-s H} A \mathrm{e}^{s H}$. Assume the following:

$$
\mathscr{U}^{*}\left(A_{j} \otimes B_{j}+\varepsilon_{j} B_{j} \otimes A_{j}\right) \mathscr{U} \unrhd 0 \quad \text { w.r.t. } \mathfrak{P}_{\mathrm{ext}}, \quad j=1, \ldots, n,
$$

where $\varepsilon_{j}=1$ or -1 . Then we have, for all $0 \leq s_{1} \leq s_{2} \leq \cdots \leq s_{n}<\beta$,

$$
\sum_{I \subseteq\{1,2, \ldots, n\}} \varepsilon_{I}\left\langle T_{I}\right\rangle_{\beta}\left\langle T_{I^{c}}\right\rangle_{\beta} \geq 0
$$

where $I^{c}=\{1,2, \ldots, n\} \backslash I, \varepsilon_{I}=\prod_{j \in I} \varepsilon_{j}$ and

$$
T_{I}=\prod_{j=1}^{n} T_{j}\left(s_{j}\right), \quad T_{j}\left(s_{j}\right)=\left\{\begin{array}{ll}
A_{j}\left(s_{j}\right) & j \in I \\
B_{j}\left(s_{j}\right) & j \in I^{c}
\end{array} .\right.
$$

In addition, assume $(\mathbf{A})$ and $(\mathbf{H} . \mathbf{1})$. If $A_{j} \unrhd 0, B_{j} \unrhd 0$ w.r.t. $\mathfrak{P}$ for all $j=1, \ldots, n$, we obtain

$$
\left\langle T_{I}\right\rangle_{\beta} \geq 0
$$

for all $0 \leq s_{1} \leq s_{2} \leq \cdots \leq s_{n}<\beta$ and $I \subseteq\{1,2, \ldots, n\}$.

Remark 2.16 Let $\left\langle\langle\cdot\rangle_{\beta}\right.$ be defined by (2.10). Then we obtain (2.29) from the following:

$$
\left\langle\left\langle\prod_{j=1}^{n}\left[A_{j}\left(s_{j}\right) \otimes B_{j}\left(s_{j}\right)+\varepsilon_{j} B_{j}\left(s_{j}\right) \otimes A_{j}\left(s_{j}\right)\right]\right\rangle_{\beta} \geq 0 .\right.
$$

Thus (2.32) can be regarded as a generalization of the second quantum Griffiths inequality as well. This expression will be useful in the later sections. $\diamond$ 
Theorem 2.17 If we replace (A) and (B) by (A') in Theorem 2.15, then (2.29) and (2.31) hold true at $\beta=\infty$.

Example 1 When $n=2$, by (2.29), we have

$$
\varepsilon_{1} \varepsilon_{2}\left\langle A_{1} A_{2}\right\rangle\left\langle B_{1} B_{2}\right\rangle+\varepsilon_{1}\left\langle A_{1} B_{2}\right\rangle\left\langle B_{1} A_{2}\right\rangle+\varepsilon_{2}\left\langle B_{1} A_{2}\right\rangle\left\langle A_{1} B_{2}\right\rangle+\left\langle B_{1} B_{2}\right\rangle\left\langle A_{1} A_{2}\right\rangle \geq 0 .
$$

Here $\left\langle A_{1} A_{2} \cdots A_{n}\right\rangle$ is an abbreviation of $\left\langle A_{1}\left(s_{1}\right) A_{2}\left(s_{2}\right) \cdots A_{n}\left(s_{n}\right)\right\rangle_{\beta}$. Thus, if $\varepsilon_{1}=\varepsilon_{2}=$ -1 , we obtain Theorem 2.12. $\diamond$

Example 2 Consider the case where $n=3$ and $B_{1}=B_{2}=B_{3}=\mathbb{1}$. In this case, (2.29) is meaningful only if $\varepsilon_{1} \varepsilon_{2} \varepsilon_{3}=1$ :

$$
\left\langle A_{1} A_{2} A_{3}\right\rangle+\varepsilon_{1}\left\langle A_{1}\right\rangle\left\langle A_{2} A_{3}\right\rangle+\varepsilon_{2}\left\langle A_{2}\right\rangle\left\langle A_{1} A_{3}\right\rangle+\varepsilon_{3}\left\langle A_{3}\right\rangle\left\langle A_{1} A_{2}\right\rangle \geq 0 .
$$

Moreover, suppose that assumption (2.28) is satisfied for

$$
\left(\varepsilon_{1}, \varepsilon_{2}, \varepsilon_{3}\right)=(1,-1,-1),(-1,1,-1),(-1,-1,1)
$$

then we obtain

$$
\begin{aligned}
& \left\langle A_{1} A_{2} A_{3}\right\rangle-\left\langle A_{1}\right\rangle\left\langle A_{2} A_{3}\right\rangle \geq 0, \\
& \left\langle A_{1} A_{2} A_{3}\right\rangle-\left\langle A_{2}\right\rangle\left\langle A_{1} A_{3}\right\rangle \geq 0, \\
& \left\langle A_{1} A_{2} A_{3}\right\rangle-\left\langle A_{3}\right\rangle\left\langle A_{1} A_{2}\right\rangle \geq 0,
\end{aligned}
$$

which implies that

$$
3\left\langle A_{1} A_{2} A_{3}\right\rangle-\left\langle A_{1}\right\rangle\left\langle A_{2} A_{3}\right\rangle-\left\langle A_{2}\right\rangle\left\langle A_{1} A_{3}\right\rangle-\left\langle A_{3}\right\rangle\left\langle A_{1} A_{2}\right\rangle \geq 0 . \quad \diamond
$$

Example 3 Consider the case where $n=4, \varepsilon_{1} \varepsilon_{2} \varepsilon_{3} \varepsilon_{4}=1$, and $B_{1}=B_{2}=B_{3}=B_{4}=$ 11. In this case, (2.29) implies that

$$
\begin{aligned}
& \left\langle A_{1} A_{2} A_{3} A_{4}\right\rangle+\varepsilon_{3} \varepsilon_{4}\left\langle A_{1} A_{2}\right\rangle\left\langle A_{3} A_{4}\right\rangle+\varepsilon_{2} \varepsilon_{4}\left\langle A_{1} A_{3}\right\rangle\left\langle A_{2} A_{4}\right\rangle+\varepsilon_{2} \varepsilon_{3}\left\langle A_{1} A_{4}\right\rangle\left\langle A_{2} A_{3}\right\rangle \\
\varepsilon_{4} & \left\langle A_{1} A_{2} A_{3}\right\rangle\left\langle A_{4}\right\rangle+\varepsilon_{3}\left\langle A_{1} A_{2} A_{4}\right\rangle\left\langle A_{3}\right\rangle+\varepsilon_{2}\left\langle A_{1} A_{3} A_{4}\right\rangle\left\langle A_{2}\right\rangle+\varepsilon_{1}\left\langle A_{2} A_{3} A_{4}\right\rangle\left\langle A_{1}\right\rangle \\
& \geq 0 .
\end{aligned}
$$

Let $S=\left\{\left(\varepsilon_{1}, \varepsilon_{2}, \varepsilon_{3}, \varepsilon_{4}\right) \in\{ \pm 1\}^{4} \mid \varepsilon_{1} \varepsilon_{2} \varepsilon_{3} \varepsilon_{4}=1\right\}$. If assumption (2.28) holds true for all $\left(\varepsilon_{1}, \varepsilon_{2}, \varepsilon_{3}, \varepsilon_{4}\right) \in S$, we obtain

$$
3\left\langle A_{1} A_{2} A_{3} A_{4}\right\rangle-\left\langle A_{1} A_{2}\right\rangle\left\langle A_{3} A_{4}\right\rangle-\left\langle A_{1} A_{3}\right\rangle\left\langle A_{2} A_{4}\right\rangle-\left\langle A_{1} A_{4}\right\rangle\left\langle A_{2} A_{3}\right\rangle \geq 0 .
$$

The following theorem offers us a connection between Corollary 2.14 and Theorem 2.15 (similar arguments can be found in [20]):

Theorem 2.18 Assume (B) and (H. 2). Let $A_{j} \in \mathscr{B}(\mathfrak{H}), j=1, \ldots, n$. Assume that

$$
\mathscr{U}^{*}\left(A_{j} \otimes \mathbb{1}+\mathbb{1} \otimes A_{j}\right) \mathscr{U} \unrhd 0, \quad \mathscr{U}^{*}\left(A_{j} \otimes \mathbb{1}-\mathbb{1} \otimes A_{j}\right) \mathscr{U} \unrhd 0 \quad \text { w.r.t. } \mathfrak{P}_{\text {ext }}
$$


for all $j=1, \ldots, n$. For each $I=\left\{i_{1}, \ldots, i_{k}\right\} \subseteq\{1, \ldots, n\}$, we set

$$
A_{I}=\prod_{\ell=1}^{k} A_{i_{\ell}}
$$

Then we obtain

$$
\left\langle A_{I} A_{K}\right\rangle_{\beta}-\left\langle A_{I}\right\rangle_{\beta}\left\langle A_{K}\right\rangle_{\beta} \geq 0
$$

for all $I, K \subseteq\{1, \ldots, n\}$.

Proof. For each $\varepsilon \in\{ \pm 1\}$, define $B_{j}^{(\varepsilon)}=\frac{1}{2}\left(A_{j} \otimes \mathbb{1}+\varepsilon \mathbb{1} \otimes A_{j}\right)$. By (2.42) , we have $B_{j}^{(\varepsilon)} \unrhd 0$ w.r.t. $\mathfrak{P}_{\text {ext }}$ for all $j=1, \ldots, n$. Since $A_{j} \otimes \mathbb{1}=B_{j}^{(+)}+B_{j}^{(-)}$and $\mathbb{1} \otimes A_{j}=B_{j}^{(+)}-B_{j}^{(-)}$, we see that

$$
\begin{aligned}
& A_{I} \otimes \mathbb{1}-\mathbb{1} \otimes A_{I}=\prod_{\ell=1}^{k}\left[B_{i_{\ell}}^{(+)}+B_{i_{\ell}}^{(-)}\right]-\overrightarrow{\prod_{\ell=1}^{k}}\left[B_{i_{\ell}}^{(+)}-B_{i_{\ell}}^{(-)}\right] \\
& =\sum_{\varepsilon_{1}, \ldots, \varepsilon_{k} \in\{ \pm 1\}} C_{\varepsilon_{1}, \ldots, \varepsilon_{k}} B_{i_{1}}^{\left(\varepsilon_{1}\right)} \cdots B_{i_{k}}^{\left(\varepsilon_{k}\right)},
\end{aligned}
$$

where $C_{\varepsilon_{1}, \ldots, \varepsilon_{k}} \geq 0$ for all $\varepsilon_{1}, \ldots, \varepsilon_{k} \in\{ \pm 1\}$. Thus, the RHS of $(2.45) \unrhd 0$ w.r.t. $\mathfrak{P}_{\text {ext }}$. Similarly, $A_{K} \otimes \mathbb{1}-\mathbb{1} \otimes A_{K} \unrhd 0$ w.r.t. $\mathfrak{P}_{\text {ext }}$. By applying Corollary 2.14, we obtain the result.

\section{Reflection positivity}

In Section 2, we give a general framework of the Griffiths inequality. In our proofs, assumptions (A) and (B) are basic inputs. Unfortunately, these assumptions are not satisfied in several models. To overcome this situation, we employ the concept of reflection positivity. As we indicated in 44, reflection positivity can be considered an operator inequality associated with a special self-dual cone. This viewpoint makes it possible to visualize a common mathematical structure among various quantum models. Reflection positivity originates from axiomatic quantum field theory [51]. Glimm, Jaffe, and Spencer first applied reflection positivity to the rigorous study of the phase transition [21]. This idea was successfully further developed by Dyson, Fröhlich, Israel, Lieb, Simon, [11, 17, 18, and many others. Lieb also discovered a crucial application of reflection positivity to many-electron systems, called the spin reflection positivity [38. Recently, Jaffe and Pedrocchi studied the topological order by reflection positivity [30, 31].

For each $p \in \mathbb{N}$, we denote the trace ideal by $\mathscr{L}^{p}(\mathfrak{H})$, which is defined as

$$
\mathscr{L}^{p}(\mathfrak{H})=\left\{\xi \in \mathscr{B}(\mathfrak{H}) \mid \operatorname{Tr}\left[|\xi|^{p}\right]<\infty\right\} .
$$

$\mathscr{L}^{1}(\mathfrak{H})$ is called the trace class, while $\mathscr{L}^{2}(\mathfrak{H})$ is called the Hilbert-Schmidt class. $\mathscr{L}^{2}(\mathfrak{H})$ becomes a Hilbert space if we define the inner product as $\langle\eta \mid \xi\rangle_{\mathscr{L}^{2}}=\operatorname{Tr}\left[\eta^{*} \xi\right]$ for all $\eta, \xi \in \mathscr{L}^{2}(\mathfrak{H})$. 
Definition 3.1 (Bounded operators) Let $A \in \mathscr{B}(\mathfrak{H})$.

(i) The left multiplication operator $\mathcal{L}(A)$ is defined as $\mathcal{L}(A) \xi=A \xi$ for all $\xi \in \mathscr{L}^{2}(\mathfrak{H})$.

(ii) The right multiplication operator $\mathcal{R}(A)$ is defined as $\mathcal{R}(A) \xi=\xi A$ for all $\xi \in$ $\mathscr{L}^{2}(\mathfrak{H}) \cdot \diamond$

Remark 3.2 (i) $\mathcal{L}(A), \mathcal{R}(A) \in \mathscr{B}\left(\mathscr{L}^{2}(\mathfrak{H})\right)$, the set of all bounded operators on $\mathscr{L}^{2}(\mathfrak{H})$.

(ii) $\mathcal{L}(A) \mathcal{L}(B)=\mathcal{L}(A B)$.

(iii) $\mathcal{R}(A) \mathcal{R}(B)=\mathcal{R}(B A) \cdot \diamond$

Let $\vartheta$ be an antilinear involution on $\mathfrak{H}$. Let $\Phi_{\vartheta}$ be an isometric isomorphism from $\mathscr{L}^{2}(\mathfrak{H})$ onto $\mathfrak{H} \otimes \mathfrak{H}$ defined by

$$
\Phi_{\vartheta}(|x\rangle\langle y|)=x \otimes \vartheta y \quad \forall x, y \in \mathfrak{H} .
$$

We have the relations

$$
\mathcal{L}(A)=\Phi_{\vartheta}^{-1} A \otimes \mathbb{1} \Phi_{\vartheta}, \quad \mathcal{R}\left(\vartheta A^{*} \vartheta\right)=\Phi_{\vartheta}^{-1} \mathbb{1} \otimes A \Phi_{\vartheta}
$$

for each $A \in \mathscr{B}(\mathfrak{H})$. We simply write these facts as

$$
\mathfrak{H} \otimes \mathfrak{H}=\mathscr{L}^{2}(\mathfrak{H}), \quad A \otimes \mathbb{1}=\mathcal{L}(A), \quad \mathbb{1} \otimes A=\mathcal{R}\left(\vartheta A^{*} \vartheta\right),
$$

if no confusion arises.

Definition 3.1 can be extended to unbounded operators by (3.3) as follows.

Definition 3.3 (Unbounded operators) Let $A$ be a densely defined closed operator on $\mathfrak{H}$.

(i) The left multiplication operator $\mathcal{L}(A)$ is defined as $\mathcal{L}(A)=\Phi_{\vartheta}^{-1} A \otimes \mathbb{1} \Phi_{\vartheta}$.

(ii) The right multiplication operator $\mathcal{R}(A)$ is defined as $\mathcal{R}(A)=\Phi_{\vartheta}^{-1} \mathbb{1} \otimes \vartheta A^{*} \vartheta \Phi_{\vartheta}$. $\diamond$

Remark 3.4 (i) Both $\mathcal{L}(A)$ and $\mathcal{R}(A)$ are closed operators on $\mathscr{L}^{2}(\mathfrak{H})$.

(ii) If $A$ is self-adjoint, so are $\mathcal{L}(A)$ and $\mathcal{R}(A)$.

(iii) We will also use the conventional identification (3.4). $\diamond$

Definition 3.5 A canonical cone in $\mathscr{L}^{2}(\mathfrak{H})$ is defined by

$$
\mathscr{L}^{2}(\mathfrak{H})_{+}=\left\{\xi \in \mathscr{L}^{2}(\mathfrak{H}) \mid \xi \geq 0 \text { as a linear operator in } \mathfrak{H}\right\} .
$$

$\mathscr{L}^{2}(\mathfrak{H})_{+}$is self-dual. $\diamond$

The following proposition is often useful.

Proposition 3.6 For each $A \in \mathscr{B}(\mathfrak{H})$, we have $\mathcal{L}(A) \mathcal{R}\left(A^{*}\right) \unrhd 0$ w.r.t. $\mathscr{L}^{2}(\mathfrak{H})_{+}$.

Proof. For each $\xi \in \mathscr{L}^{2}(\mathfrak{H})_{+}$, we can see that $\mathcal{L}(A) \mathcal{R}\left(A^{*}\right) \xi=A \xi A^{*} \geq 0$. 
Definition 3.7 We define

$$
\mathfrak{A}=\operatorname{Coni}\left\{\mathcal{L}(A) \mathcal{R}\left(A^{*}\right) \in \mathscr{B}\left(\mathscr{L}^{2}(\mathfrak{H})\right) \mid A \in \mathscr{B}(\mathfrak{H})\right\}^{-\mathrm{w}},
$$

where $\operatorname{Coni}(X)$ is the conical hull of $X$ and $S^{- \text {w }}$ represents the closure of $S$ under a weak topology in $\mathscr{B}\left(\mathscr{L}^{2}(\mathfrak{H})\right)$.

If $A \in \mathfrak{A}$, then we write $A \succeq 0$ w.r.t. $\mathscr{L}^{2}(\mathfrak{H})_{+} \cdot \diamond$

Remark $3.8 \quad$ (i) $A \succeq 0 \Longrightarrow A \unrhd 04$

(ii) $A \succeq 0, B \succeq 0, a, b \geq 0 \Longrightarrow a A+b B \succeq 0$.

(iii) $A \succeq 0, B \succeq 0 \Longrightarrow A B \succeq 0 . \diamond$

The following proposition is a guiding principle of reflection positivity [11, 17, 44]. The point is that assumptions (A) and (B) are unnecessary.

Proposition 3.9 (Reflection positivity) Assume that $A$ is a trace class operator on $\mathscr{L}^{2}(\mathfrak{H})$, i.e., $A \in \mathscr{L}^{1}\left(\mathscr{L}^{2}(\mathfrak{H})\right)$. If $A \succeq 0$ w.r.t. $\mathscr{L}^{2}(\mathfrak{H})_{+}$, then we have $\operatorname{Tr}_{\mathscr{L}^{2}}[A] \geq 0$.

Proof. It suffices to consider the case where $A=\sum_{j=1}^{N} \mathcal{L}\left(a_{j}\right) \mathcal{R}\left(a_{j}^{*}\right), N \in \mathbb{N}$. In this case, we can easily see that $\operatorname{Tr}_{\mathscr{L}^{2}}[A]=\sum_{j=1}^{N}\left|\operatorname{Tr}_{\mathfrak{H}}\left[a_{j}\right]\right|^{2} \geq 0$.

As before, the system's Hamiltonian $H$ is a self-adjoint operator acting in $\mathscr{L}^{2}(\mathfrak{H})$ and bounded from below. In this section, we continue to assume that $\mathrm{e}^{-\beta H}$ is a trace class operator for all $\beta>0$. Corresponding to (H. 1), we need the following condition:

(H. 3) $\mathrm{e}^{-\beta H} \succeq 0$ w.r.t. $\mathscr{L}^{2}(\mathfrak{H})_{+}$for all $\beta \geq 0$.

Let $\langle\cdot\rangle_{\beta}$ be the thermal average. Theorem 3.10 is another prototype of the Griffiths inequality.

Theorem 3.10 Assume (H. 3). If $A \succeq 0$ w.r.t. $\mathscr{L}^{2}(\mathfrak{H})_{+}$, then $\langle A\rangle_{\beta} \geq 0$ for all $\beta \geq 0$.

Proof. From Remark 3.8 (iii), we have $A \mathrm{e}^{-\beta H} \succeq 0$ w.r.t. $\mathscr{L}^{2}(\mathfrak{H})_{+}$for all $\beta \geq 0$. Thus by Proposition 3.9, we conclude the theorem.

Theorem 3.10 can be generalized as follows.

Theorem 3.11 Assume (H. 3). If $A_{j} \succeq 0$ w.r.t. $\mathscr{L}^{2}(\mathfrak{H})_{+}$for all $j=1, \ldots, n$, then we have

$$
\left\langle\prod_{j=1}^{\stackrel{n}{\longrightarrow}} A_{j}\left(s_{j}\right)\right\rangle_{\beta} \geq 0
$$

for all $0 \leq s_{1} \leq s_{2} \leq \cdots \leq s_{n}<\beta$.

\footnotetext{
${ }^{4}$ From this fact, we understand that reflection positivity is closely related to the notion of positivity preservation discussed in Section 2
} 
Proof. Since

$$
\left[\prod_{j=1}^{n} A_{j}\left(s_{j}\right)\right] \mathrm{e}^{-\beta H}=\underbrace{\mathrm{e}^{-s_{1} H}}_{\succeq 0} \underbrace{A_{1}}_{\succeq 0} \underbrace{\mathrm{e}^{-\left(s_{2}-s_{1}\right) H}}_{\succeq 0} \cdots \underbrace{A_{n}}_{\succeq 0} \underbrace{\mathrm{e}^{-\left(\beta-s_{n}\right) H}}_{\succeq 0} \succeq 0
$$

w.r.t. $\mathscr{L}^{2}(\mathfrak{H})_{+}$, we obtain (3.7) by Proposition 3.9

Theorem 3.12 Assume (A'). Then Theorem 3.11 holds true at $\beta=\infty$.

Proof. Considering Remark 3.8 (i), we know that Theorem 3.12 follows from Theorem 2.8 .

\section{$4 \quad$ Quantum Ising model}

\subsection{Results}

Let $\Lambda$ be a finite subset of $\mathbb{R}^{d}$. The Hamiltonian of the quantum Ising model is given by

$$
H_{\Lambda}=-\sum_{x, y \in \Lambda} J_{x y} \sigma_{x}^{(3)} \sigma_{y}^{(3)}-\sum_{x \in \Lambda} \mu_{x} \sigma_{x}^{(3)}-\sum_{x \in \Lambda} \lambda_{x} \sigma_{x}^{(1)}
$$

$\sigma^{(1)}, \sigma^{(2)}$, and $\sigma^{(3)}$ are the Pauli matrices:

$$
\sigma^{(1)}=\left(\begin{array}{ll}
0 & 1 \\
1 & 0
\end{array}\right), \quad \sigma^{(2)}=\left(\begin{array}{ll}
0 & -i \\
i & 0
\end{array}\right), \quad \sigma^{(3)}=\left(\begin{array}{ll}
1 & 0 \\
0 & -1
\end{array}\right) .
$$

$H_{\Lambda}$ acts in the Hilbert space $\mathfrak{H}_{\Lambda}=\otimes_{x \in \Lambda} \mathbb{C}^{2} .\left(J_{x y}\right)_{x, y \in \mathbb{Z}^{d}}$ is a family of coupling constants, and $\mu_{x}, \lambda_{x} \in \mathbb{R}$ are the magnetic fields. In this section, we always assume the following:

$$
J_{x y} \geq 0, \quad J_{x y}=J_{y x}, \quad J_{x x}=0 .
$$

The thermal average is defined by

$$
\langle A\rangle_{\beta}=\operatorname{Tr}\left[A \mathrm{e}^{-\beta H_{\Lambda}}\right] / Z_{\beta}, \quad Z_{\beta}=\operatorname{Tr}\left[\mathrm{e}^{-\beta H_{\Lambda}}\right] .
$$

Let

$$
\tau_{x}=\frac{1}{2}\left(1+\sigma_{x}^{(1)}\right)
$$

Set

$$
S_{x}^{(\varepsilon)}=\left\{\begin{array}{ll}
\tau_{x} & \text { if } \varepsilon=1 \\
\sigma_{x}^{(3)} & \text { if } \varepsilon=3
\end{array}\right. \text {. }
$$

We define

$$
\mathfrak{A}=\operatorname{Coni}\left\{S_{x_{1}}^{\left(\varepsilon_{1}\right)} \cdots S_{x_{n}}^{\left(\varepsilon_{n}\right)} \mid x_{1}, \ldots, x_{n} \in \Lambda, \varepsilon_{1}, \ldots, \varepsilon_{n} \in\{1,3\}, n \in \mathbb{N}\right\},
$$

where $\operatorname{Coni}(S)$ is the conical hull of $S$. 
Theorem 4.1 (First Griffiths inequality) Assume (J). Assume that $\mu_{x} \geq 0$ for all $x \in \Lambda$. For all $A_{1}, \ldots, A_{n} \in \mathfrak{A}, \lambda_{x} \in \mathbb{R}$ and $0 \leq s_{1} \leq \cdots \leq s_{n} \leq \beta$, we have

$$
\left\langle\prod_{j=1}^{\stackrel{n}{\longrightarrow}} A_{j}\left(s_{j}\right)\right\rangle_{\beta} \geq 0
$$

For each $A \subseteq \Lambda$, set

$$
\sigma_{A}^{(3)}=\prod_{x \in A} \sigma_{x}^{(3)}, \quad \tau_{A}=\prod_{x \in A} \tau_{x}
$$

To state the second Griffiths inequality, we introduce the following notations:

$$
\begin{aligned}
\langle\langle X\rangle\rangle_{\beta} & =\operatorname{Tr}_{\mathfrak{H} \otimes \mathfrak{H}}\left[X \mathrm{e}^{-\beta H_{\mathrm{ext}}}\right] / Z_{\beta}^{2}, \\
H_{\mathrm{ext}} & =H_{\Lambda} \otimes \mathbb{1}+\mathbb{1} \otimes H_{\Lambda} .
\end{aligned}
$$

Theorem 4.2 (Second Griffiths inequality) Assume (J). Assume that $\mu_{x} \geq 0, \lambda_{x} \geq$ 0 for all $x \in \Lambda$. For all $A, B, C, D \subseteq \Lambda$ and $\beta \geq 0$, we have

$$
\left\langle\left\langle\left(\sigma_{A}^{(3)}(s) \otimes \tau_{C}(s)-\tau_{C}(s) \otimes \sigma_{A}^{(3)}(s)\right)\left(\sigma_{B}^{(3)}(t) \otimes \tau_{D}(t)-\tau_{D}(t) \otimes \sigma_{B}^{(3)}(t)\right)\right\rangle\right\rangle_{\beta} \geq 0
$$

for all $0 \leq s \leq t \leq \beta$, where $\sigma_{A}^{(3)}(t)=\mathrm{e}^{-t H_{\Lambda}} \sigma_{A}^{(3)} \mathrm{e}^{t H_{\Lambda}}$ and $\tau_{B}(t)=\mathrm{e}^{-t H_{\Lambda}} \tau_{B} \mathrm{e}^{t H_{\Lambda}}$.

Remark 4.3 (4.11) can be expressed as follows:

$$
\left\langle\sigma_{A}^{(3)}(s) \sigma_{B}^{(3)}(t)\right\rangle_{\beta}\left\langle\tau_{C}(s) \tau_{D}(t)\right\rangle_{\beta}-\left\langle\sigma_{A}^{(3)}(s) \tau_{D}(t)\right\rangle_{\beta}\left\langle\tau_{C}(s) \sigma_{B}^{(3)}(t)\right\rangle_{\beta} \geq 0 .
$$

From this theorem (or (4.12)), we can derive the well-known formula.

Corollary 4.4 Under the same assumptions as Theorem 4.2, we have

$$
\left\langle\sigma_{A}^{(3)} \sigma_{B}^{(3)}\right\rangle_{\beta}-\left\langle\sigma_{A}^{(3)}\right\rangle_{\beta}\left\langle\sigma_{B}^{(3)}\right\rangle_{\beta} \geq 0, \quad\left\langle\tau_{A} \tau_{B}\right\rangle_{\beta}-\left\langle\tau_{A}\right\rangle_{\beta}\left\langle\tau_{B}\right\rangle_{\beta} \geq 0
$$

The following theorem is an extension of Theorem 4.2 ,

Theorem 4.5 Assume (J). Assume that $\mu_{x} \geq 0, \lambda_{x} \geq 0$ for all $x \in \Lambda$. Let $A_{1}, \ldots, A_{n}, B_{1}, \ldots, B_{n} \subseteq$ $\Lambda$. Then, for all $0 \leq t_{1} \leq t_{2} \leq \cdots \leq t_{n} \leq \beta$, we have

$$
\left\langle\left\langle\prod_{j=1}^{n}\left(\sigma_{A_{j}}^{(3)}\left(t_{j}\right) \otimes \tau_{B_{j}}\left(t_{j}\right)-\tau_{B_{j}}\left(t_{j}\right) \otimes \sigma_{A_{j}}^{(3)}\left(t_{j}\right)\right)\right\rangle_{\beta} \geq 0 .\right.
$$

By Theorem 2.15, we obtain the following corollary. 
Corollary 4.6 Assume (J). Assume that $\mu_{x} \geq 0, \lambda_{x} \geq 0$ for all $x \in \Lambda$. Let $A_{1}, \ldots, A_{n}, B_{1}, \ldots, B_{n} \subseteq$ $\Lambda$. For each $I=\left\{i_{1}, \ldots, i_{k}\right\} \subseteq\{1, \ldots, n\}$ with $i_{1}<i_{2}<\cdots<i_{k}$, we define

$$
S_{I}(\mathbf{t})=\overrightarrow{\prod_{j=1}^{k}} S_{j}\left(t_{j}\right), \quad S_{j}\left(t_{j}\right)=\left\{\begin{array}{ll}
\sigma_{A_{j}}^{(3)}\left(t_{j}\right) & \text { if } j \in I \\
\tau_{B_{j}}\left(t_{j}\right) & \text { if } j \in I^{c}
\end{array} .\right.
$$

Then we have, for all $0 \leq t_{1} \leq t_{2} \leq \cdots \leq t_{n} \leq \beta$,

$$
\sum_{I \subseteq\{1,2, \ldots, n\}}(-1)^{|I|}\left\langle S_{I}(\mathbf{t})\right\rangle_{\beta}\left\langle S_{I^{c}}(\mathbf{t})\right\rangle_{\beta} \geq 0 .
$$

In addition, we have

$$
\left\langle S_{I}(\mathbf{t})\right\rangle_{\beta} \geq 0
$$

for all $0 \leq t_{1} \leq t_{2} \leq \cdots \leq t_{n} \leq \beta$ and $I \subseteq \Lambda$.

Example 4 We have the following:

(i) $\left\langle\sigma_{A}^{(3)}\right\rangle_{\beta}$ is monotonically increasing in $J_{x y}$ and $\mu_{x}$

(ii) $\left\langle\tau_{A}\right\rangle_{\beta}$ is monotonically decreasing in $J_{x y}$ and $\mu_{x}$.

(iii) $\left\langle\sigma_{A}^{(3)}\right\rangle_{\beta}$ is monotonically decreasing in $\lambda_{x}$.

(iv) $\left\langle\tau_{A}\right\rangle_{\beta}$ is monotonically increasing in $\lambda_{x}$.

We will prove this example in Section 4.4. $\diamond$

Remark 4.7 (i) Our results can be extended to a more general Hamiltonian of the form

$$
H_{\Lambda}=-\sum_{A \subseteq \Lambda} J_{A} \sigma_{A}^{(3)}-\sum_{A \subseteq \Lambda} K_{A} \tau_{A}
$$

with $J_{A} \geq 0$ and $K_{A} \geq 0$.

(ii) Assume that $\mu_{x}>0$ or $\lambda_{x}>0$ for all $x \in \Lambda$. Then since the ground state of $H_{\Lambda}$ is unique for all $\Lambda$, our results are valid at $\beta=\infty$. The results at $\beta=\infty$ are used in the study of quantum phase transitions $9,10 . \diamond$

\footnotetext{
${ }^{5}$ This fact can be proven by the Perron-Frobenius-Faris theorem [12].
} 


\subsection{Proof of Theorem 4.1}

Let $\Omega=\{-1,+1\}$ be the set of possible values of a spin. Given $\Lambda, \Omega^{\Lambda}$ is the set of spin configurations in $\Lambda$. Set

$$
|+1\rangle=\left(\begin{array}{l}
1 \\
0
\end{array}\right), \quad|-1\rangle=\left(\begin{array}{l}
0 \\
1
\end{array}\right)
$$

For each $\boldsymbol{\omega}=\left\{\omega_{x}\right\}_{x \in \Lambda} \in \Omega^{\Lambda}$, we define

$$
|\boldsymbol{\omega}\rangle=\bigotimes_{x \in \Lambda}\left|\omega_{x}\right\rangle
$$

Then $\left\{|\boldsymbol{\omega}\rangle \mid \boldsymbol{\omega} \in \Omega^{\Lambda}\right\}$ is a CONS of $\mathfrak{H}_{\Lambda}$.

Definition 4.8 A standard self-dual cone in $\mathfrak{H}_{\Lambda}$ is defined by

$$
\left.\mathfrak{H}_{\Lambda,+}=\left\{\Psi \in \mathfrak{H}_{\Lambda}\left|\Psi=\sum_{\boldsymbol{\omega} \in \Omega^{\Lambda}} C_{\boldsymbol{\omega}}\right| \boldsymbol{\omega}\right\rangle, \quad C_{\boldsymbol{\omega}} \geq 0 \forall \boldsymbol{\omega} \in \Omega^{\Lambda}\right\} .
$$

Remark $4.9|\boldsymbol{\omega}\rangle \in \mathfrak{H}_{\Lambda,+}$ for all $\boldsymbol{\omega} \in \Omega^{\Lambda}$. $\diamond$

Let $U$ be a unitary operator 6 on $\mathfrak{H}_{\Lambda}$ given by

$$
U=\bigotimes_{x \in \Lambda} u, \quad u=\frac{1}{\sqrt{2}}\left(\begin{array}{ll}
1 & 1 \\
-1 & 1
\end{array}\right)
$$

Since $u^{*} \sigma^{(3)} u=\sigma^{(1)}$ and $u^{*} \sigma^{(1)} u=-\sigma^{(3)}$, we have

$$
U^{*} \sigma_{x}^{(3)} U=\sigma_{x}^{(1)}, \quad U^{*} \sigma_{x}^{(1)} U=-\sigma_{x}^{(3)}
$$

for all $x \in \Lambda$. Thus,

$$
\hat{H}_{\Lambda}=U^{*} H_{\Lambda} U=-\sum_{x, y \in \Lambda} J_{x y} \sigma_{x}^{(1)} \sigma_{y}^{(1)}-\sum_{x \in \Lambda} \mu_{x} \sigma_{x}^{(1)}+\sum_{x \in \Lambda} \lambda_{x} \sigma_{x}^{(3)} .
$$

Proposition 4.10 We have the following:

(i) $\sigma_{x}^{(1)} \unrhd 0$ w.r.t. $\mathfrak{H}_{\Lambda,+}$ for all $x \in \Lambda$.

(ii) $\frac{1}{2}\left(\mathbb{1}-\sigma_{x}^{(3)}\right) \unrhd 0$ w.r.t. $\mathfrak{H}_{\Lambda,+}$ for all $x \in \Lambda$.

(iii) $\mathrm{e}^{-\beta \hat{H}_{\Lambda}} \unrhd 0$ w.r.t. $\mathfrak{H}_{\Lambda,+}$ for all $\beta \geq 0$.

Proof. (i), (ii) Let $r$ be a map on $\Omega$ defined by $r(-1)=+1$ and $r(+1)=-1$. Clearly, $\sigma^{(1)}|\omega\rangle=|r(\omega)\rangle$ holds. Then $\sigma_{x}^{(1)}|\boldsymbol{\omega}\rangle=\left|r_{x}(\boldsymbol{\omega})\right\rangle$, where $\left(r_{x}(\boldsymbol{\omega})\right)_{y}=r\left(\omega_{x}\right)$ if $y=x$, $\left(r_{x}(\boldsymbol{\omega})\right)_{y}=\omega_{y}$ if $y \neq x$. Thus, for all $\boldsymbol{\omega} \in \Omega^{\Lambda}$, it holds that $\sigma_{x}^{(1)}|\boldsymbol{\omega}\rangle \in \mathfrak{H}_{\Lambda,+}$. Thus, we conclude (i). (ii) is obvious.

\footnotetext{
${ }^{6}$ This unitary operator is well-known 34,43$]$.
} 
(iii) Let

$$
\hat{T}=\sum_{x, y \in \Lambda} J_{x y} \sigma_{x}^{(1)} \sigma_{y}^{(1)}, \quad \hat{V}_{\mu}=-\sum_{x \in \Lambda} \mu_{x} \sigma_{x}^{(1)}, \quad \hat{V}_{\lambda}=\sum_{x \in \Lambda} \lambda_{x} \sigma_{x}^{(3)}
$$

Set $\hat{V}=\hat{V}_{\mu}+\hat{V}_{\lambda}$. Then we have $\hat{H}_{\Lambda}=-\hat{T}+\hat{V}$. By (i), we have $\hat{T} \unrhd 0$ w.r.t. $\mathfrak{H}_{\Lambda,+}$. On the other hand, since $-\hat{V}_{\mu} \unrhd 0$ w.r.t. $\mathfrak{H}_{\Lambda,+}$, , we have $\mathrm{e}^{-\beta \hat{V}_{\mu}} \unrhd 0$ w.r.t. $\mathfrak{H}_{\Lambda,+}$ for all $\beta \geq 0$ by Proposition A.3. In addition, we have

$$
\mathrm{e}^{-\beta \hat{V}_{\lambda}}|\boldsymbol{\omega}\rangle=\underbrace{\exp \left\{-\beta \sum_{x \in \Lambda} \lambda_{x} \omega_{x}\right\}}_{\geq 0}|\boldsymbol{\omega}\rangle \in \mathfrak{H}_{\Lambda,+},
$$

which implies $\mathrm{e}^{-\beta \hat{V}_{\lambda}} \unrhd 0$ w.r.t. $\mathfrak{H}_{\Lambda,+}$. By Proposition $₫$ A.4, we have $\mathrm{e}^{-\beta \hat{V}} \unrhd 0$ w.r.t. $\mathfrak{H}_{\Lambda,+}$ for all $\beta \geq 0$. Now we can apply Proposition A.5 with $A=-\hat{V}, B=\hat{T}$.

\section{Proof of Theorem 4.1}

For each $x \in \Lambda$, by Proposition 4.10 (i) and (ii), we have

$$
\hat{\sigma}_{x}^{(3)}=U^{*} \sigma_{x}^{(3)} U=\sigma_{x}^{(1)} \unrhd 0, \quad \hat{\tau}_{x}=U^{*} \tau_{x} U=\frac{1}{2}\left(\mathbb{1}-\sigma_{x}^{(3)}\right) \unrhd 0
$$

w.r.t. $\mathfrak{H}_{\Lambda,+}$. Thus, $U^{*} S_{x_{1}}^{\left(\varepsilon_{1}\right)} \cdots S_{x_{n}}^{\left(\varepsilon_{n}\right)} U \unrhd 0$ w.r.t. $\mathfrak{H}_{\Lambda,+}$, implying that $\hat{A}=U^{*} A U \unrhd 0$ w.r.t. $\mathfrak{H}_{\Lambda,+}$ for all $A \in \mathfrak{A}$. By applying Theorem 2.7, we conclude Theorem 4.1 .

\subsection{Proof of Theorems 4.2 and 4.5}

\subsubsection{Preliminaries}

Let $\mathfrak{K}=\mathbb{C}^{2} \otimes \mathbb{C}^{2}=\mathbb{C}^{4}$. Then $\left\{\left|\omega, \omega^{\prime}\right\rangle \mid \omega, \omega^{\prime} \in \Omega\right\}$ is a CONS of $\mathfrak{K}$, where $\left|\omega, \omega^{\prime}\right\rangle=$ $|\omega\rangle \otimes\left|\omega^{\prime}\right\rangle$. We label $\left\{\left|\omega, \omega^{\prime}\right\rangle \mid \omega, \omega^{\prime} \in \Omega\right\}$ as

$$
\left|e^{1}\right\rangle=|+1,+1\rangle, \quad\left|e^{2}\right\rangle=|-1,-1\rangle, \quad\left|e^{3}\right\rangle=|+1,-1\rangle, \quad\left|e^{4}\right\rangle=|-1,+1\rangle .
$$

Thus, each $|\varphi\rangle=\sum_{j=1}^{4} c_{j}\left|e_{j}\right\rangle \in \mathfrak{K}$ can be identified with $\left(c_{1}, c_{2}, c_{3}, c_{4}\right)^{T} \in \mathbb{C}^{4}$. We introduce linear operators on $\mathfrak{K}$ as

$$
\begin{aligned}
& \psi=\frac{1}{\sqrt{2}}\left(\sigma^{(3)} \otimes \mathbb{1}+\mathbb{1} \otimes \sigma^{(3)}\right), \\
& \phi=\frac{1}{\sqrt{2}}\left(\sigma^{(3)} \otimes \mathbb{1}-\mathbb{1} \otimes \sigma^{(3)}\right), \\
& \eta=\frac{1}{\sqrt{2}}\left(\sigma^{(1)} \otimes \mathbb{1}+\mathbb{1} \otimes \sigma^{(1)}\right), \\
& \xi=\frac{1}{\sqrt{2}}\left(\sigma^{(1)} \otimes \mathbb{1}-\mathbb{1} \otimes \sigma^{(1)}\right) .
\end{aligned}
$$

\footnotetext{
${ }^{7}$ We used the assumption $\mu_{x} \geq 0$ here.
} 
In general, each operator $X$ in $\mathfrak{K}$ can be expressed as a $4 \times 4$ matrix: $X=\left(X_{i j}\right)_{i, j=1,2,3,4}$ with $X_{i j}=\left\langle e_{i} \mid X e_{j}\right\rangle$. In particular, we have

$$
\begin{aligned}
& \psi=\sqrt{2}\left(\begin{array}{ll}
\sigma^{(3)} & 0 \\
0 & 0
\end{array}\right), \quad \phi=\sqrt{2}\left(\begin{array}{ll}
0 & 0 \\
0 & \sigma^{(3)}
\end{array}\right), \\
& \eta=\sqrt{2}\left(\begin{array}{ll}
\sigma^{(1)} & \alpha \\
\alpha & \sigma^{(1)}
\end{array}\right), \quad \xi=\sqrt{2}\left(\begin{array}{ll}
0 & \gamma \\
\gamma & 0
\end{array}\right),
\end{aligned}
$$

where

$$
\alpha=\frac{1}{\sqrt{2}}\left(\begin{array}{ll}
1 & 1 \\
1 & 1
\end{array}\right), \quad \gamma=\frac{1}{\sqrt{2}}\left(\begin{array}{ll}
-1 & 1 \\
1 & -1
\end{array}\right) .
$$

Let $u$ be the unitary operator given by (4.22) and let

$$
\vartheta=\left(\begin{array}{ll}
u & 0 \\
0 & u
\end{array}\right) \text {. }
$$

For each operator $X$ on $\mathfrak{K}$, we write $\tilde{X}=\vartheta^{*} X \vartheta$. By (4.33), we obtain

$$
\begin{aligned}
& \tilde{\psi}=\sqrt{2}\left(\begin{array}{ll}
\sigma^{(1)} & 0 \\
0 & 0
\end{array}\right), \quad \tilde{\phi}=\sqrt{2}\left(\begin{array}{ll}
0 & 0 \\
0 & \sigma^{(1)}
\end{array}\right), \\
& \tilde{\eta}=\sqrt{2}\left(\begin{array}{ll}
-\sigma^{(3)} & \hat{\alpha} \\
\hat{\alpha} & -\sigma^{(3)}
\end{array}\right), \quad \tilde{\xi}=\sqrt{2}\left(\begin{array}{ll}
0 & \hat{\gamma} \\
\hat{\gamma} & 0
\end{array}\right)
\end{aligned}
$$

where $\hat{\alpha}=u^{*} \alpha u=\left(\mathbb{1}_{2}-\sigma^{(3)}\right) / \sqrt{2}$ and $\hat{\gamma}=-\left(\mathbb{1}_{2}+\sigma^{(3)}\right) / \sqrt{2}$.

\section{Definition 4.11 Let}

$$
\left.\mathfrak{K}_{+}=\{|\varphi\rangle \in \mathfrak{K}|| \varphi\rangle=\sum_{j=1}^{4} c_{j}\left|e_{j}\right\rangle, \quad c_{j} \geq 0, j=1,2,3,4\right\} .
$$

Clearly, $\mathfrak{K}_{+}$is a self-dual cone in $\mathfrak{K} . \diamond$

Proposition 4.12 We have the following:

(i) $\tilde{\psi} \unrhd 0$ w.r.t. $\mathfrak{K}_{+}$.

(ii) $\tilde{\phi} \unrhd 0$ w.r.t. $\mathfrak{K}_{+}$.

(iii) $-\tilde{\xi} \unrhd 0$ w.r.t. $\mathfrak{K}_{+}$.

(iv) $\mathbb{1}_{4}+\frac{1}{\sqrt{2}} \tilde{\eta} \unrhd 0$ w.r.t. $\mathfrak{K}_{+}$.

(v) $\exp (\beta \tilde{\psi}) \unrhd 0$ w.r.t. $\mathfrak{K}_{+}$for all $\beta \geq 0$.

(vi) $\exp (\beta \tilde{\eta}) \unrhd 0$ w.r.t. $\mathfrak{K}_{+}$for all $\beta \geq 0$. 
Proof. Note that by Proposition A.2, a linear operator $X$ on $\mathfrak{K}$ satisfies $X \unrhd 0$ w.r.t. $\mathfrak{K}_{+}$if and only if $X_{i j}=\left\langle e_{i} \mid X e_{j}\right\rangle \geq 0$ for all $i, j=1,2,3,4$. Thus, (i), (ii), (iii), and (iv) immediately follow from (4.36).

(v) By (i) and Proposition A.3, we can see that $\exp (\beta \tilde{\psi}) \unrhd 0$ w.r.t. $\mathfrak{K}_{+}$for all $\beta \geq 0$. To show (vi), we write $\tilde{\eta}=\tilde{\eta}_{d}+\tilde{\eta}_{o}$, where

$$
\tilde{\eta}_{d}=\sqrt{2}\left(\begin{array}{ll}
-\sigma^{(3)} & 0 \\
0 & -\sigma^{(3)}
\end{array}\right), \quad \tilde{\eta}_{o}=\sqrt{2}\left(\begin{array}{ll}
0 & \hat{\alpha} \\
\hat{\alpha} & 0
\end{array}\right) .
$$

Suppose that

(a) $\exp \left(\beta \tilde{\eta}_{d}\right) \unrhd 0$ w.r.t. $\mathfrak{K}_{+}$for all $\beta \geq 0$,

(b) $\exp \left(\beta \tilde{\eta}_{o}\right) \unrhd 0$ w.r.t. $\mathfrak{K}_{+}$for all $\beta \geq 0$.

Then we can immediately conclude (vi) by Proposition A.4. Hence, it suffices to prove (a) and (b).

To show (a), observe that

$$
\exp \left(\beta \tilde{\eta}_{d}\right)=\left(\begin{array}{ll}
\mathrm{e}^{-\sqrt{2} \beta \sigma^{(3)}} & 0 \\
0 & \mathrm{e}^{-\sqrt{2} \beta \sigma^{(3)}}
\end{array}\right) .
$$

Since all matrix elements of $\exp \left(-\sqrt{2} \beta \sigma^{(3)}\right)$ are positive, we conclude that (a) is true by Proposition A.2.

Since $\tilde{\eta}_{o} \unrhd 0$ w.r.t. $\mathfrak{K}_{+}$, we find that $\exp \left(\beta \tilde{\eta}_{o}\right) \unrhd 0$ w.r.t. $\mathfrak{K}_{+}$for all $\beta \geq 0$ by Proposition A.3. Thus, we conclude (b).

\subsubsection{Completion of proof of Theorems 4.2 and 4.5}

Let $H_{\text {ext }}$ be given by (4.10). $H_{\text {ext }}$ acts in the extended Hilbert space $\mathfrak{K}_{\Lambda}=\mathfrak{H}_{\Lambda} \otimes \mathfrak{H}_{\Lambda}$. For each $x \in \Lambda$, let

$$
\begin{aligned}
& \psi_{x}=\frac{1}{\sqrt{2}}\left(\sigma_{x}^{(3)} \otimes \mathbb{1}+\mathbb{1} \otimes \sigma_{x}^{(3)}\right), \\
& \phi_{x}=\frac{1}{\sqrt{2}}\left(\sigma_{x}^{(3)} \otimes \mathbb{1}-\mathbb{1} \otimes \sigma_{x}^{(3)}\right), \\
& \eta_{x}=\frac{1}{\sqrt{2}}\left(\sigma_{x}^{(1)} \otimes \mathbb{1}+\mathbb{1} \otimes \sigma_{x}^{(1)}\right), \\
& \xi_{x}=\frac{1}{\sqrt{2}}\left(\sigma_{x}^{(1)} \otimes \mathbb{1}-\mathbb{1} \otimes \sigma_{x}^{(1)}\right) .
\end{aligned}
$$

$H_{\text {ext }}$ can be expressed as

$$
H_{\mathrm{ext}}=-\sum_{x, y \in \Lambda} J_{x y}\left(\psi_{x} \psi_{y}+\phi_{x} \phi_{y}\right)-\sqrt{2} \sum_{x \in \Lambda} \mu_{x} \psi_{x}-\sqrt{2} \sum_{x \in \Lambda} \lambda_{x} \eta_{x}
$$

We employ the following identification 8 of $\mathfrak{K}_{\Lambda}$ :

$$
\mathfrak{K}_{\Lambda}=\bigotimes_{x \in \Lambda} \mathfrak{K},
$$

\footnotetext{
${ }^{8}$ Indeed, we have $\mathfrak{K}_{\Lambda}=\left(\bigotimes_{x \in \Lambda} \mathbb{C}^{2}\right) \otimes\left(\bigotimes_{x \in \Lambda} \mathbb{C}^{2}\right) \cong \bigotimes_{x \in \Lambda}\left(\mathbb{C}^{2} \otimes \mathbb{C}^{2}\right) \cong \bigotimes_{x \in \Lambda} \mathfrak{K}$.
} 
where $\mathfrak{K}=\mathbb{C}^{4}$. Thus, $\psi_{x}, \phi_{x}$, and $\eta_{x}$ can be expressed as

$$
\psi_{x}=\otimes_{y \in \Lambda}(\psi)^{\delta_{x y}}, \quad \phi_{x}=\otimes_{y \in \Lambda}(\phi)^{\delta_{x y}}, \quad \eta_{x}=\otimes_{y \in \Lambda}(\eta)^{\delta_{x y}} .
$$

Here $(X)^{\delta_{x y}}=\mathbb{1}$ if $x \neq y,(X)^{\delta_{x y}}=X$ if $x=y$. Let $\vartheta$ be given by (4.35). Set $\Theta=\otimes_{x \in \Lambda} \vartheta$. For each linear operator $X$ on $\mathfrak{K}_{\Lambda}$, set $\tilde{X}=\Theta^{*} X \Theta$. Then we obtain

$$
\tilde{H}_{\mathrm{ext}}=-\sum_{x, y \in \Lambda} J_{x y}\left(\tilde{\psi}_{x} \tilde{\psi}_{y}+\tilde{\phi}_{x} \tilde{\phi}_{y}\right)-\sqrt{2} \sum_{x \in \Lambda} \mu_{x} \tilde{\psi}_{x}-\sqrt{2} \sum_{x \in \Lambda} \lambda_{x} \tilde{\eta}_{x},
$$

where $\tilde{\psi}_{x}, \tilde{\phi}_{x}, \tilde{\eta}_{x}, \tilde{\xi}_{x}$ are defined through (4.36) and (4.46).

Definition 4.13 We define a self-dual cone in $\mathfrak{K}_{\Lambda}$ by

$$
\left.\mathfrak{K}_{\Lambda,+}:=\left\{|\Psi\rangle \in \mathfrak{K}_{\Lambda}|| \Psi\right\rangle=\sum_{\mathbf{n} \in\{1,2,3,4\}^{\Lambda}} C_{\mathbf{n}}\left|e_{\mathbf{n}}\right\rangle, C_{\mathbf{n}} \geq 0 \forall \mathbf{n} \in\{1,2,3,4\}^{\Lambda}\right\},
$$

where $\left|e_{\mathbf{n}}\right\rangle=\otimes_{x \in \Lambda}\left|e_{n_{x}}\right\rangle$ for each $\mathbf{n}=\left\{n_{x}\right\}_{x \in \Lambda} \in\{1,2,3,4\}^{\Lambda} . \diamond$

Remark 4.14 $\left|e_{\mathbf{n}}\right\rangle \in \mathfrak{K}_{\Lambda,+}$ for all $\mathbf{n} \in\{1,2,3,4\}^{\Lambda} . \diamond$

Proposition 4.15 We have the following:

(i) $\tilde{\psi}_{x} \unrhd 0$ w.r.t. $\mathfrak{K}_{\Lambda,+}$ for all $x \in \Lambda$.

(ii) $\tilde{\phi}_{x} \unrhd 0$ w.r.t. $\mathfrak{K}_{\Lambda,+}$ for all $x \in \Lambda$.

(iii) $-\tilde{\xi}_{x} \unrhd 0$ w.r.t. $\mathfrak{K}_{\Lambda,+}$ for all $x \in \Lambda$.

(iv) $\mathbb{1}+\frac{1}{\sqrt{2}} \tilde{\eta}_{x} \unrhd 0 \mathfrak{K}_{\Lambda,+}$ for all $x \in \Lambda$.

(v) $\exp \left(\beta \tilde{\psi}_{x}\right) \unrhd 0$ w.r.t. $\mathfrak{K}_{\Lambda,+}$ for all $x \in \Lambda$ and $\beta \geq 0$.

(vi) $\exp \left(\beta \tilde{\eta}_{x}\right) \unrhd 0$ w.r.t. $\mathfrak{K}_{\Lambda,+}$ for all $x \in \Lambda$ and $\beta \geq 0$.

Proof. By Proposition A.2, a linear operator $X$ in $\mathfrak{K}_{\Lambda}$ satisfies $X \unrhd 0$ w.r.t. $\mathfrak{K}_{\Lambda,+}$ if and only if $\left\langle e_{\mathbf{n}} \mid X e_{\mathbf{m}}\right\rangle \geq 0$ for all $\mathbf{m}, \mathbf{n} \in\{1,2,3,4\}^{\Lambda}$. Thus, the assertions immediately follow from Proposition 4.12 .

Corollary $4.16 \exp \left(-\beta \tilde{H}_{\text {ext }}\right) \unrhd 0$ w.r.t. $\mathfrak{K}_{\Lambda,+}$ for all $\beta \geq 0$.

Proof. Set $\tilde{H}_{\text {ext }}=-\tilde{T}+\tilde{V}$, where

$$
\tilde{T}=\sum_{x, y \in \Lambda} J_{x y}\left(\tilde{\psi}_{x} \tilde{\psi}_{y}+\tilde{\phi}_{x} \tilde{\phi}_{y}\right), \quad \tilde{V}=\tilde{V}_{\mu}+\tilde{V}_{\lambda}
$$

with $\tilde{V}_{\mu}=-\sqrt{2} \sum_{x \in \Lambda} \mu_{x} \tilde{\psi}_{x}$ and $\tilde{V}_{\lambda}=-\sqrt{2} \sum_{x \in \Lambda} \lambda_{x} \tilde{\eta}_{x}$. By Proposition 4.15 (i) and (ii), it holds that $\tilde{T} \unrhd 0$ w.r.t. $\mathfrak{K}_{\Lambda,+}$. On the other hand, we can see that by Proposition $4.15(\mathrm{v})$ and (vi),

$$
\mathrm{e}^{-\beta V_{\mu}}=\prod_{x \in \Lambda} \underbrace{\mathrm{e}^{\sqrt{2} \beta \mu_{x} \tilde{\psi}_{x}}}_{\unrhd 0} \unrhd 0, \quad \mathrm{e}^{-\beta V_{\lambda}}=\prod_{x \in \Lambda} \underbrace{\mathrm{e}^{\sqrt{2} \beta \lambda_{x} \tilde{\eta}_{x}}}_{\unrhd 0} \unrhd 0
$$

w.r.t. $\mathfrak{K}_{\Lambda,+}$ for all $\beta \geq 09$ Thus, by Proposition $₫$ A.4, we obtain $\mathrm{e}^{-\beta \tilde{V}} \unrhd 0$ w.r.t. $\mathfrak{K}_{\Lambda,+}$ for all $\beta \geq 0$. By applying Proposition $A .5$, we conclude the desired assertion.

\footnotetext{
${ }^{9}$ Here we have used the assumptions $\mu_{x} \geq 0$ and $\lambda_{x} \geq 0$.
} 
Corollary 4.17 For all $A, B, C, D \subseteq \Lambda$, we have the following:

(i) $\Theta^{*} \sigma_{A}^{(3)} \otimes \tau_{C} \Theta \unrhd 0$ w.r.t. $\mathfrak{K}_{\Lambda,+}$.

(ii) $\Theta^{*}\left(\sigma_{B}^{(3)} \otimes \tau_{D}-\tau_{D} \otimes \sigma_{B}^{(3)}\right) \Theta \unrhd 0$ w.r.t. $\mathfrak{K}_{\Lambda,+}$.

Proof. (i) Let $\tilde{\ell}_{x}=\frac{1}{2}\left(1+\frac{1}{\sqrt{2}} \tilde{\eta}_{x}\right)$ and $\tilde{m}_{x}=-\frac{1}{2 \sqrt{2}} \tilde{\xi}_{x}$. By Proposition 4.15 (iii) and (iv), it holds that $\tilde{\ell}_{x} \unrhd 0$ and $\tilde{m}_{x} \unrhd 0$ w.r.t. $\mathfrak{K}_{\Lambda,+}$. Thus, we have

$$
\Theta^{*} \sigma_{A}^{(3)} \otimes \tau_{C} \Theta=2^{-|A| / 2} \prod_{x \in A}\left(\tilde{\psi}_{x}+\tilde{\phi}_{x}\right) \prod_{x \in C}\left(\tilde{\ell}_{x}+\tilde{m}_{x}\right) \unrhd 0
$$

w.r.t. $\mathfrak{K}_{\Lambda,+}$ by Proposition 4.15,

(ii) We have

$$
\begin{aligned}
& \Theta^{*}\left(\sigma_{B}^{(3)} \otimes \tau_{D}-\tau_{D} \otimes \sigma_{B}^{(3)}\right) \Theta \\
= & 2^{-|B| / 2} \prod_{x \in B} \prod_{y \in D}\left(\tilde{\psi}_{x}+\tilde{\phi}_{x}\right)\left(\tilde{\ell}_{y}+\tilde{m}_{y}\right)-2^{-|B| / 2} \prod_{x \in B} \prod_{y \in D}\left(\tilde{\psi}_{x}-\tilde{\phi}_{x}\right)\left(\tilde{\ell}_{y}-\tilde{m}_{y}\right) \\
= & \sum_{X_{1}, X_{2} \subset B} \sum_{Y_{1}, Y_{2} \subset D} K_{X_{1} X_{2} Y_{1} Y_{2}} \tilde{\psi}_{X_{1}} \tilde{\phi}_{X_{2}} \tilde{\ell}_{Y_{1}} \tilde{m}_{Y_{2}}
\end{aligned}
$$

with $K_{X_{1} X_{2} Y_{1} Y_{2}} \geq 0, \tilde{\psi}_{X_{1}}=\prod_{x \in X_{1}} \tilde{\psi}_{x}, \tilde{\phi}_{X_{2}}=\prod_{x \in X_{2}} \tilde{\phi}_{x}, \tilde{\ell}_{Y_{1}}=\prod_{x \in Y_{1}} \tilde{\ell}_{x}, \quad \tilde{m}_{Y_{2}}=$ $\prod_{x \in Y_{2}} \tilde{m}_{x}$. Thus, $\Theta^{*}\left(\sigma_{B}^{(3)} \otimes \tau_{D}-\tau_{D} \otimes \sigma_{B}^{(3)}\right) \Theta \unrhd 0$ w.r.t. $\mathfrak{K}_{\Lambda,+}$.

Proof of Theorems 4.2 and 4.5

By Corollaries 4.16, 4.17 and Theorem A.1, we have

$$
\begin{aligned}
& \left\langle\left\langle\left(\sigma_{A}^{(3)}(s) \otimes \tau_{C}(s)-\tau_{C}(s) \otimes \sigma_{A}^{(3)}(s)\right)\left(\sigma_{B}^{(3)}(t) \otimes \tau_{D}(t)-\tau_{D}(t) \otimes \sigma_{B}^{(3)}(t)\right)\right\rangle\right\rangle_{\beta} \\
& =Z_{\beta}^{-2} \operatorname{Tr}[\underbrace{\mathrm{e}^{-s \tilde{H}_{\mathrm{ext}}}}_{\unrhd 0} \underbrace{\Theta^{*}\left(\sigma_{A}^{(3)} \otimes \tau_{C}-\tau_{C} \otimes \sigma_{A}^{(3)}\right)}_{\unrhd 0} \Theta \underbrace{\underbrace{-(t-s) \tilde{H}_{\mathrm{ext}}}}_{\unrhd 0} \\
& \times \underbrace{\Theta^{*}\left(\sigma_{B}^{(3)} \otimes \tau_{D}-\tau_{D} \otimes \sigma_{B}^{(3)}\right)}_{\unrhd 0} \Theta \underbrace{e^{-(\beta-t) \tilde{H}_{\mathrm{ext}}}}_{\unrhd 0}] \geq 0 .
\end{aligned}
$$

This concludes Theorem 4.2 .

Similarly, we can show Theorem 4.5 by Corollary 4.17 and Theorem A.1.

\subsection{Proof of Example 4}

We only prove (i) and (ii), since (iii) and (iv) can be proved in a similar manner.

Recall the Duhamel formula

$$
\mathrm{e}^{-t(A+B)}=\sum_{n \geq 0} \int_{0 \leq t_{1} \leq t_{2} \leq \cdots \leq t_{n} \leq \beta}\left(-B\left(t_{1}\right)\right) \cdots\left(-B\left(t_{n}\right)\right) \mathrm{e}^{-t A} d t_{1} \cdots d t_{n}
$$


for any bounded self-adjoint operators $A$ and $B$ with $B(t)=\mathrm{e}^{-t A} B \mathrm{e}^{t A}$. Using this, we have

$$
\begin{aligned}
\frac{\partial}{\partial J_{x y}} \mathrm{e}^{-\beta H} & =\sum_{n \geq 1} D_{n}, \\
D_{n} & =n\left(J_{x y}\right)^{n-1} \int_{0 \leq t_{1} \leq \cdots \leq t_{n} \leq \beta} T_{x y}\left[t_{1}\right] \cdots T_{x y}\left[t_{n}\right] \mathrm{e}^{-\beta H^{\prime}} d t_{1} \cdots d t_{n},
\end{aligned}
$$

where $T_{x y}=\sigma_{x}^{(3)} \sigma_{y}^{(3)}, H^{\prime}=H_{\Lambda}+J_{x y} \sigma_{x}^{(3)} \sigma_{y}^{(3)}$ and $T_{x y}[t]=\mathrm{e}^{-t H^{\prime}} T_{x y} \mathrm{e}^{t H^{\prime}}$. Note that in a similar manner to Sections 4.2 and 4.3 , we have the following:

(a) $\mathrm{e}^{-\beta \hat{H}^{\prime}} \unrhd 0$ w.r.t. $\mathfrak{H}_{\Lambda,+}$ for all $\beta \geq 0$, where $\hat{H}^{\prime}=U^{*} H^{\prime} U$.

(b) $\mathrm{e}^{-\beta \tilde{H}_{\mathrm{ext}}^{\prime}} \unrhd 0$ w.r.t. $\mathfrak{K}_{\Lambda,+}$ for all $\beta \geq 0$, where $H_{\mathrm{ext}}^{\prime}=H^{\prime} \otimes \mathbb{1}+\mathbb{1} \otimes H^{\prime}$.

Hence, by setting $\mathrm{M}_{n}=T_{x y}\left[t_{1}\right] \cdots T_{x y}\left[t_{n}\right]$, we obtain

$$
\begin{aligned}
& \frac{\partial}{\partial J_{x y}}\left\langle\sigma_{A}^{(3)}\right\rangle_{\beta} \\
= & \sum_{n \geq 1} n\left(J_{x y}\right)^{n-1} \int_{0 \leq t_{1} \leq \cdots \leq \beta}\left\{\left\langle\sigma_{A}^{(3)} \mathrm{M}_{n}\right\rangle_{H^{\prime}, \beta}-\left\langle\sigma_{A}^{(3)}\right\rangle_{H^{\prime}, \beta}\left\langle\mathrm{M}_{n}\right\rangle_{H^{\prime}, \beta}\right\} d t_{1} \cdots d t_{n} \\
= & \sum_{n \geq 1} \frac{n\left(J_{x y}\right)^{n-1}}{2} \int_{0 \leq t_{1} \leq \cdots \leq \beta}\left\langle\left\langle\left(\sigma_{A}^{(3)} \otimes \mathbb{1}-\mathbb{1} \otimes \sigma_{A}^{(3)}\right)\left(\mathrm{M}_{n} \otimes \mathbb{1}-\mathbb{1} \otimes \mathrm{M}_{n}\right)\right\rangle\right\rangle_{H^{\prime}, \beta} d t_{1} \cdots d t_{n} \\
& \geq 0
\end{aligned}
$$

where $\langle\cdot\rangle_{H^{\prime}, \beta}$ and $\langle\langle\cdot\rangle\rangle_{H^{\prime}, \beta}$ are the thermal averages associated with $H^{\prime}$ and $H_{\text {ext }}^{\prime}$. (Here we used the facts that $\Theta^{*}\left(\sigma_{A}^{(3)} \otimes \mathbb{1}-\mathbb{1} \otimes \sigma_{A}^{(3)}\right) \Theta \unrhd 0$ and $\Theta^{*}\left(\mathrm{M}_{n} \otimes \mathbb{1}-\mathbb{1} \otimes \mathrm{M}_{n}\right) \mathrm{e}^{-\beta H_{\text {ext }}^{\prime}} \Theta \unrhd 0$ w.r.t. $\mathfrak{K}_{\Lambda,+}$, which follow from Corollary 4.17) Thus, we have proved (i). Similarly, by applying the fact that $\Theta^{*}\left(\tau_{A} \otimes \mathbb{1}-\mathbb{1} \otimes \tau_{A}\right) \Theta \unlhd 0$ w.r.t. $\mathfrak{K}_{\Lambda,+}$, which follows from Corollary 4.17, we have

$$
\begin{aligned}
& \frac{\partial}{\partial J_{x y}}\left\langle\tau_{A}\right\rangle_{\beta} \\
= & \sum_{n \geq 1} \frac{n\left(J_{x y}\right)^{n-1}}{2} \int_{0 \leq t_{1} \leq \cdots \leq \beta}\left\langle\left\langle\left(\tau_{A} \otimes \mathbb{1}-\mathbb{1} \otimes \tau_{A}\right)\left(\mathrm{M}_{n} \otimes \mathbb{1}-\mathbb{1} \otimes \mathrm{M}_{n}\right)\right\rangle\right\rangle_{H^{\prime}, \beta} d t_{1} \cdots d t_{n} \\
& \leq 0 .
\end{aligned}
$$

Thus, we have proved (ii).

\section{Quantum rotor model}

\section{$5.1 \quad$ Results}

Let $\Lambda$ be a finite subset of $\mathbb{R}^{2}$. The quantum rotor model on $\Lambda$ is defined by

$$
H=\sum_{x \in \Lambda} \frac{U_{x}}{2}\left(-i \frac{\partial}{\partial \theta_{x}}\right)^{2}-\sum_{x, y \in \Lambda} t_{x y} \cos \left(\theta_{x}-\theta_{y}\right) .
$$


The Hilbert space is $\mathfrak{H}=\otimes_{x \in \Lambda} L^{2}(\mathbb{T})$ with $\mathbb{T}=[-\pi, \pi] . \quad U_{x}>0$ being the strength of the on site repulsion and $t_{x y} \geq 0$ being the hopping strength. $H$ is a self-adjoint operator acting in the Hilbert space $\mathfrak{H} 10$ We refer readers who want to learn the physical background to [6, 54].

Remark 5.1 In this study, we simply write $M_{f}$, the multiplication operator by the function $f$, as $f(\theta)$ if no confusion occurs. $\diamond$

Let $T_{x}=\mathrm{e}^{i \theta_{x}}$. For each $A=\left\{m_{x}\right\}_{x \in \Lambda} \in \mathbb{Z}^{\Lambda}$, we set

$$
T^{A}=\prod_{x \in \Lambda}\left(T_{x}\right)^{m_{x}}
$$

Let

$$
\mathfrak{A}=\operatorname{Coni}\left\{T^{A} \mid A \in \mathbb{Z}^{\Lambda}\right\}^{-\mathrm{w}} .
$$

The thermal expectation value $\langle\cdot\rangle_{\beta}$ is defined by

$$
\langle A\rangle_{\beta}=\operatorname{Tr}\left[A \mathrm{e}^{-\beta H}\right] / Z_{\beta}, \quad Z_{\beta}=\operatorname{Tr}\left[\mathrm{e}^{-\beta H}\right]
$$

for all $A \in \mathscr{B}(\mathfrak{H})$.

Theorem 5.2 (First Griffiths inequality) Let $A_{1}, \ldots, A_{n} \in \mathfrak{A}$. For all $0 \leq s_{1} \leq$ $s_{2} \leq \cdots \leq s_{n}<\beta$, we have

$$
\left\langle\prod_{j=1}^{\stackrel{n}{\longrightarrow}} A_{j}\left(s_{j}\right)\right\rangle_{\beta} \geq 0
$$

To state second Griffiths inequality, some conditions are required. We introduce an extended Hilbert space $\mathfrak{H}_{\text {ext }}$ by $\mathfrak{H}_{\text {ext }}=\mathfrak{H} \otimes \mathfrak{H}$. For each $X \in \mathscr{B}\left(\mathfrak{H}_{\text {ext }}\right)$, we set

$$
\begin{aligned}
\langle\langle X\rangle\rangle_{\beta} & =\operatorname{Tr}_{\mathfrak{H}_{\text {ext }}}\left[X \mathrm{e}^{-\beta H_{\text {ext }}}\right] / Z_{\beta}^{2}, \\
H_{\text {ext }} & =H \otimes \mathbb{1}+\mathbb{1} \otimes H .
\end{aligned}
$$

Let $C_{x}=\cos \theta_{x}$ and

$$
C_{x}(s)=\mathrm{e}^{-s H} C_{x} \mathrm{e}^{s H} .
$$

Theorem 5.3 (Second Griffiths inequality) For all $x_{1}, \ldots, x_{n} \in \Lambda, 0 \leq s_{1} \leq s_{2} \leq$ $\cdots \leq s_{n}<\beta$ and $\varepsilon_{1}, \ldots, \varepsilon_{n} \in\{ \pm 1\}$, we have

$$
\left\langle\left\langle\prod_{j=1}^{n}\left[C_{x_{j}}\left(s_{j}\right) \otimes \mathbb{1}+\varepsilon_{j} \mathbb{1} \otimes C_{x_{j}}\left(s_{j}\right)\right]\right\rangle\right\rangle_{\beta} \geq 0 .
$$

\footnotetext{
${ }^{10}$ The precise definition of $-i \frac{\partial}{\partial \theta}$ is given by

$$
\begin{aligned}
\operatorname{dom}\left(-i \frac{\partial}{\partial \theta}\right) & =\left\{f \in C^{1}(\mathbb{T}) \mid f(-\pi)=f(\pi)\right\}, \\
-i \frac{\partial}{\partial \theta} f & =-i f^{\prime} \quad \forall f \in \operatorname{dom}\left(-i \frac{\partial}{\partial \theta}\right) .
\end{aligned}
$$
}

Then $-i \frac{\partial}{\partial \theta}$ is essentially self-adjoint. We still denote its closure by the same symbol. 
From Theorem [5.3, we immediately obtain Corollary [5.4, which has a form similar to (1.4). (This is why we call Theorem 5.3 the second Griffiths inequality, see Remark 2.16 and Theorem 2.18 for general arguments.)

Corollary 5.4 For each $A=\left\{m_{x}\right\}_{x \in \Lambda} \in \mathbb{N}^{\Lambda}$, set

$$
C^{A}=\prod_{x \in \Lambda}\left(C_{x}\right)^{m_{x}} .
$$

For all $A, B \in \mathbb{N}^{\Lambda}$, we obtain

$$
\left\langle C^{A} C^{B}\right\rangle_{\beta} \geq\left\langle C^{A}\right\rangle_{\beta}\left\langle C^{B}\right\rangle_{\beta} .
$$

Let

$$
n_{x}=-i \frac{\partial}{\partial \theta_{x}} .
$$

Set $n_{x}(s)=\mathrm{e}^{-s H} n_{x} \mathrm{e}^{s H}$. We have the following.

Theorem 5.5 For all $x_{1}, \ldots, x_{n} \in \Lambda, 0 \leq s_{1} \leq s_{2} \leq \cdots \leq s_{n}<\beta$ and $\varepsilon_{1}, \ldots, \varepsilon_{n} \in$ $\{ \pm 1\}$, we have

$$
\left\langle\left\langle\stackrel{n}{\prod_{j=1}^{n}}\left[n_{x_{j}}\left(s_{j}\right) \otimes \mathbb{1}+\varepsilon_{j} \mathbb{1} \otimes n_{x_{j}}\left(s_{j}\right)\right]\left[n_{x_{j}}\left(s_{j}\right) \otimes \mathbb{1}+\bar{\varepsilon}_{j} \mathbb{1} \otimes n_{x_{j}}\left(s_{j}\right)\right]\right\rangle_{\beta} \geq 0,\right.
$$

where $\bar{\varepsilon}_{j}=-\varepsilon_{j}$.

We can construct several extensions of Theorems 5.3 and 5.5 . Theorem 5.6 illustrates this fact. Let

$$
\begin{aligned}
& \alpha_{x}^{(1)}(s)=C_{x}(s) \otimes \mathbb{1}+\mathbb{1} \otimes C_{x}(s), \\
& \alpha_{x}^{(2)}(s)=C_{x}(s) \otimes \mathbb{1}-\mathbb{1} \otimes C_{x}(s), \\
& \alpha_{x}^{(3)}(s)=\left[n_{x}(s) \otimes \mathbb{1}+\mathbb{1} \otimes n_{x}(s)\right]\left[n_{x}(s) \otimes \mathbb{1}-\mathbb{1} \otimes n_{x}(s)\right] .
\end{aligned}
$$

Theorem 5.6 For all $x_{1}, \ldots, x_{n} \in \Lambda, \mu_{1}, \ldots, \mu_{n} \in\{1,2,3\}$ and $0 \leq s_{1} \leq s_{2} \leq \cdots \leq$ $s_{n} \leq \beta$, we have

$$
\left\langle\left\langle\prod_{j=1}^{n} \alpha_{x_{j}}^{\left(\mu_{j}\right)}\left(s_{j}\right)\right\rangle\right\rangle_{\beta} \geq 0 .
$$

Example 5 For all $A \subseteq \Lambda$ and $x, y, z \in \Lambda$, we have the following:

(i) $\left\langle C^{A}\right\rangle_{\beta}$ is monotonically increasing in $t_{x y}$.

(ii) $\left\langle n_{z}^{2}\right\rangle_{\beta}$ is monotonically increasing in $t_{x y}$.

(iii) $\left.\frac{\partial}{\partial U_{x}}\left\langle C^{A}\right\rangle_{\beta}\right|_{U_{x}=0} \leq 0$. 
We will provide a proof of this example in Section 5.4. $\diamond$

Remark 5.7 (i) Our results can be extended to a more general Hamiltonian of the form

$$
H=\sum_{x \in \Lambda} \frac{U_{x}}{2}\left(-i \frac{\partial}{\partial \theta_{x}}\right)^{2}-\sum_{A \in \mathbb{N}^{\Lambda}} J_{A} C^{A}
$$

with $J_{A} \geq 0$, where the sum converges under a uniform topology.

(ii) Since the ground state of $H$ is unique, our results are valid at $\beta=\infty$. The results at $\beta=\infty$ are essential for the study of quantum phase transitions [36]. $\diamond$

\subsection{Proof of Theorem 5.2}

Let $\mathcal{F}$ be the Fourier transformation 11 on $\mathfrak{H}$ and let $\hat{H}=\mathcal{F} H \mathcal{F}^{-1}$. We have

$$
\hat{H}=\sum_{x \in \Lambda} \frac{U_{x}}{2} \hat{n}_{x}^{2}+\frac{1}{2} \sum_{x, y \in \Lambda}\left(-t_{x y}\right)\left(\hat{T}_{x} \hat{T}_{y}^{*}+\hat{T}_{x}^{*} \hat{T}_{y}\right) .
$$

$\hat{H}$ acts in the Hilbert space $\hat{\mathfrak{H}}=\mathcal{F} \mathfrak{H}=\otimes_{x \in \Lambda} \ell^{2}(\mathbb{Z}) . \quad \hat{n}_{x}$ and $\hat{T}_{x}$ are defined by $\hat{n}_{x}=$ $\mathcal{F} n_{x} \mathcal{F}^{-1}$ and $\hat{T}_{x}=\mathcal{F} T_{x} \mathcal{F}^{-1}$.

For each $n \in \mathbb{Z}$, set $e_{n}(m)=\delta_{m n} \in \ell^{2}(\mathbb{Z})$. $\left\{e_{n} \mid n \in \mathbb{Z}\right\}$ is a CONS in $\ell^{2}(\mathbb{Z})$. For each $\mathbf{n}=\left\{n_{x}\right\}_{x \in \Lambda} \in \mathbb{Z}^{\Lambda}$, let $e_{\mathbf{n}}=\otimes_{x \in \Lambda} e_{n_{x}}$. Clearly, $\left\{e_{\mathbf{n}} \mid \mathbf{n} \in \mathbb{Z}^{\Lambda}\right\}$ is a CONS of $\hat{\mathfrak{H}}$ as well. Remarkably, for each $\mathbf{n}=\left\{n_{x}\right\}_{x \in \Lambda} \in \mathbb{Z}^{\Lambda}$,

$$
\hat{n}_{x} e_{\mathbf{n}}=n_{x} e_{\mathbf{n}}, \quad \hat{T}_{x} e_{\mathbf{n}}=e_{\mathbf{n}+\boldsymbol{\delta}_{x}}
$$

where $\boldsymbol{\delta}_{x}=\left\{\delta_{x y}\right\}_{y \in \Lambda} \in \mathbb{Z}^{\Lambda}$. In other words, $\hat{n}_{x}$ is the number operator and $\hat{T}_{x}$ is the creation operator at site $x$.

Definition 5.8 Let

$$
\hat{\mathfrak{H}}_{+}=\left\{F=\sum_{\mathbf{n} \in \mathbb{Z}^{\Lambda}} F(\mathbf{n}) e_{\mathbf{n}} \in \hat{\mathfrak{H}} \mid F(\mathbf{n}) \geq 0 \quad \forall \mathbf{n} \in \mathbb{Z}^{\Lambda}\right\} .
$$

Note that $\hat{\mathfrak{H}}_{+}$is a self-dual cone in $\hat{\mathfrak{H}}$. Clearly, $e_{\mathbf{n}} \in \hat{\mathfrak{H}}_{+}$for all $\mathbf{n} \in \mathbb{Z}^{\Lambda} . \diamond$

Proposition 5.9 We have the following:

(i) $\hat{T}_{x} \unrhd 0$ w.r.t. $\hat{\mathfrak{H}}_{+}$for all $x \in \Lambda$.

(ii) $\mathrm{e}^{-\beta \hat{H}} \unrhd 0$ w.r.t. $\hat{\mathfrak{H}}_{+}$for all $\beta \geq 0$.

\footnotetext{
${ }^{11}$ To be precise, $\mathcal{F}$ is a unitary operator given by$$
(\mathcal{F} f)(\mathbf{n})=(2 \pi)^{-|\Lambda| / 2} \int_{\mathbb{T}^{\Lambda}} f(\boldsymbol{\theta}) \mathrm{e}^{-i \boldsymbol{\theta} \cdot \mathbf{n}} d \boldsymbol{\theta} \quad \forall f \in \mathfrak{H} .
$$ 
Proof. (i) Note that $\hat{\mathfrak{H}}_{+}=\operatorname{Coni}\left\{e_{\mathbf{n}} \mid \mathbf{n} \in \mathbb{Z}^{\Lambda}\right\}^{-}$, where $\operatorname{Coni}(S)^{-}$is the closure of $\operatorname{Coni}(S)$. Thus, it suffices to show that $\hat{T}_{x} e_{\mathbf{n}} \geq 0$ w.r.t. $\hat{\mathfrak{H}}_{+}$for all $\mathbf{n} \in \mathbb{Z}^{\Lambda}$. This is trivial according to (5.21).

(ii) Let

$$
-\hat{\mathbf{K}}=\frac{1}{2} \sum_{x, y \in \Lambda} t_{x y}\left(\hat{T}_{x} \hat{T}_{y}^{*}+\hat{T}_{x}^{*} \hat{T}_{y}\right), \quad \hat{\mathbf{U}}=\sum_{x \in \Lambda} \frac{U_{x}}{2} \hat{n}_{x}^{2} .
$$

By (i), we can see that $-\hat{\mathbf{K}} \unrhd 0$ w.r.t. $\hat{\mathfrak{H}}_{+}$. On the other hand, since

$$
\mathrm{e}^{-\beta \hat{\mathbf{U}}} e_{\mathbf{n}}=\underbrace{\exp \left\{-\beta \sum_{x \in \Lambda} \frac{U_{x}}{2} n_{x}^{2}\right\}}_{\geq 0} e_{\mathbf{n}} \text { for all } \mathbf{n}=\left\{n_{x}\right\} \in \mathbb{Z}^{\Lambda},
$$

we have $\mathrm{e}^{-\beta \hat{\mathbf{U}}} \unrhd 0$ w.r.t. $\hat{\mathfrak{H}}_{+}$. Thus, by Proposition A.5, we conclude (ii).

\subsubsection{Completion of proof of Theorem 5.2}

By Proposition 5.9 (i), we have $A \unrhd 0$ w.r.t. $\hat{\mathfrak{H}}_{+}$for all $\mathfrak{A}$. Applying Theorem 2.7, we prove Theorem 5.2 .

\subsection{Proof of Theorems 5.3 , 5.5, and 5.6 and Corollary 5.4}

First, note the following identification:

$$
\mathfrak{H}_{\text {ext }}=L^{2}\left(\mathbb{T}^{\Lambda} \times \mathbb{T}^{\Lambda}, d \boldsymbol{\theta} d \boldsymbol{\theta}^{\prime}\right) .
$$

Under the identification (5.25), we see that

$$
\begin{aligned}
H_{\mathrm{ext}}= & H \otimes \mathbb{1}+\mathbb{1} \otimes H \\
= & \sum_{x \in \Lambda} \frac{U_{x}}{2}\left\{\left(-i \frac{\partial}{\partial \theta_{x}}\right)^{2}+\left(-i \frac{\partial}{\partial \theta_{x}^{\prime}}\right)^{2}\right\} \\
& -\sum_{x, y \in \Lambda} t_{x y}\left\{\cos \left(\theta_{x}-\theta_{y}\right)+\cos \left(\theta_{x}^{\prime}-\theta_{y}^{\prime}\right)\right\} .
\end{aligned}
$$

Next, we introduce a new coordinate system $\left\{\phi_{x}, \phi_{x}^{\prime}\right\}$ with

$$
\phi_{x}=\frac{1}{2}\left(\theta_{x}^{\prime}-\theta_{x}\right), \quad \phi_{x}^{\prime}=\frac{1}{2}\left(\theta_{x}^{\prime}+\theta_{x}\right) .
$$

Then we easily see that

$$
\mathfrak{H}_{\mathrm{ext}}=L^{2}\left(\mathbb{T}^{\Lambda} \times \mathbb{T}^{\Lambda}, d \phi d \phi^{\prime}\right)
$$

Using the identity

$$
\cos \theta+\cos \theta^{\prime}=2 \cos \frac{\theta^{\prime}+\theta}{2} \cos \frac{\theta^{\prime}-\theta}{2},
$$


we obtain

$$
H_{\mathrm{ext}}=\sum_{x \in \Lambda} \frac{U_{x}}{4}\left(\nu_{x}^{2}+\nu_{x}^{\prime 2}\right)-2 \sum_{x, y \in \Lambda} t_{x y} \cos \left(\phi_{x}-\phi_{y}\right) \cos \left(\phi_{x}^{\prime}-\phi_{y}^{\prime}\right),
$$

where

$$
\nu_{x}=-i \frac{\partial}{\partial \phi_{x}}, \quad \nu_{x}^{\prime}=-i \frac{\partial}{\partial \phi_{x}^{\prime}} .
$$

Let $\mathfrak{X}=L^{2}\left(\mathbb{T}^{\Lambda}, d \phi\right)$. Then by (5.28), we obtain the following identification:

$$
\mathfrak{H}_{\text {ext }}=L^{2}\left(\mathbb{T}^{\Lambda}, d \phi\right) \otimes L^{2}\left(\mathbb{T}^{\Lambda}, d \phi\right)=\mathfrak{X} \otimes \mathfrak{X} .
$$

Moreover, we obtain the following proposition.

Proposition 5.10 We have $H_{\mathrm{ext}}=\mathrm{T}-\mathrm{V}$, where

$$
\begin{aligned}
& \mathrm{T}=\sum_{x \in \Lambda} \frac{U_{x}}{4}\left(\nu_{x}^{2} \otimes \mathbb{1}+\mathbb{1} \otimes \nu_{x}^{2}\right), \\
& \mathrm{V}=2 \sum_{x, y \in \Lambda} t_{x y} \cos \left(\phi_{x}-\phi_{y}\right) \otimes \cos \left(\phi_{x}-\phi_{y}\right) .
\end{aligned}
$$

Let $\vartheta$ be the antilinear isomorphism defined by

$$
(\vartheta f)(\phi)=\bar{f}(\phi) \text { a.e., } \quad f \in L^{2}\left(\mathbb{T}^{\Lambda}, d \phi\right) .
$$

By (3.4) and (5.32), we have the identification $\mathfrak{H}_{\text {ext }}=\mathscr{L}^{2}(\mathfrak{X})$ by $\vartheta$. Moreover, by (3.4), we have the following proposition:

Proposition 5.11 We have $H_{\mathrm{ext}}=\mathrm{T}-\mathrm{V}$, where

$$
\begin{aligned}
\mathrm{T} & =\sum_{x \in \Lambda} \frac{U_{x}}{4}\left\{\mathcal{L}\left(\nu_{x}^{2}\right)+\mathcal{R}\left(\nu_{x}^{2}\right)\right\} \\
\mathrm{V} & =2 \sum_{x, y \in \Lambda} t_{x y} \mathcal{L}\left[\cos \left(\phi_{x}-\phi_{y}\right)\right] \mathcal{R}\left[\cos \left(\phi_{x}-\phi_{y}\right)\right] .
\end{aligned}
$$

By Corollary A.9, we immediately obtain the following:

Corollary 5.12 We have $\exp \left(-\beta H_{\text {ext }}\right) \succeq 0$ w.r.t. $\mathscr{L}^{2}(\mathfrak{X})_{+}$for all $\beta \geq 0$.

\subsubsection{Completion of proof of Theorem 5.3 and Corollary 5.4}

Proposition 5.13 We have the following:

(i) $\cos \theta_{x} \otimes \mathbb{1}+\mathbb{1} \otimes \cos \theta_{x}=2 \mathcal{L}\left(\cos \phi_{x}\right) \mathcal{R}\left(\cos \phi_{x}\right) \succeq 0$ w.r.t. $\mathscr{L}^{2}(\mathfrak{X})_{+}$.

(ii) $\cos \theta_{x} \otimes \mathbb{1}-\mathbb{1} \otimes \cos \theta_{x}=2 \mathcal{L}\left(\sin \phi_{x}\right) \mathcal{R}\left(\sin \phi_{x}\right) \succeq 0$ w.r.t. $\mathscr{L}^{2}(\mathfrak{X})_{+}$. 
Proof. (i), (ii) We apply Ginibre's idea [20]:

$$
\begin{aligned}
& \cos a+\cos b=2 \cos \frac{b+a}{2} \cos \frac{b-a}{2}, \\
& \cos a-\cos b=2 \sin \frac{b+a}{2} \sin \frac{b-a}{2} .
\end{aligned}
$$

Put

$$
2 V_{x}^{(\varepsilon)}=C_{x} \otimes \mathbb{1}+\varepsilon \mathbb{1} \otimes C_{x}, \quad \varepsilon= \pm 1 .
$$

Then by Proposition 5.13, we have $V_{x}^{(\varepsilon)} \succeq 0$ w.r.t. $\mathscr{L}^{2}(\mathfrak{X})_{+}$for all $x \in \Lambda$ and $\varepsilon \in\{ \pm 1\}$. Since $\exp \left(-\beta H_{\text {ext }}\right) \succeq 0$ w.r.t. $\mathscr{L}^{2}(\mathfrak{X})_{+}$for all $\beta \geq 0$ by Corollary 5.12 , we can apply Theorem 3.11. Thus, we conclude Theorem 5.3.

For each $\mathcal{A} \subseteq \Lambda$, define $[\mathcal{A}]=\left\{m_{x}\right\}_{x \in \Lambda} \in\{0,1\}^{\Lambda}$ by $m_{x}=1$ if $x \in \mathcal{A}$ and $m_{x}=0$ otherwise. For simplicity, we will consider the case where $A=[\mathcal{A}]$ and $B=[\mathcal{B}]$. To prove Corollary 5.4, we note

$$
C_{x} \otimes \mathbb{1}=V_{x}^{(+1)}+V_{x}^{(-1)}, \quad \mathbb{1} \otimes C_{x}=V_{x}^{(+1)}-V_{x}^{(-1)} .
$$

Observe that

$$
\begin{aligned}
& 2\left\langle C^{A} C^{B}\right\rangle_{\beta}-2\left\langle C^{A}\right\rangle_{\beta}\left\langle C^{B}\right\rangle_{\beta} \\
= & \left\langle\left\langle\left(C^{A} \otimes \mathbb{1}-\mathbb{1} \otimes C^{A}\right)\left(C^{B} \otimes \mathbb{1}-\mathbb{1} \otimes C^{B}\right)\right\rangle\right\rangle_{\beta} \\
= & \sum_{\mathcal{X} \subseteq \mathcal{A}} \sum_{\mathcal{Y} \subseteq \mathcal{B}} \underbrace{\left[1-(-1)^{|\mathcal{X}|}\right]\left[1-(-1)^{|\mathcal{Y}|}\right]}_{\geq 0} \underbrace{\left\langle\left\langle V_{\mathcal{A} \backslash \mathcal{X}}^{(+1)} V_{\mathcal{X}}^{(-1)} V_{\mathcal{B} \backslash \mathcal{Y}}^{(+1)} V_{\mathcal{Y}}^{(-1)}\right\rangle\right\rangle_{\beta}}_{\geq 0 \text { by Theorem }[5.3} \geq 0,
\end{aligned}
$$

where $V_{\mathcal{A}}^{( \pm 1)}=\prod_{x \in \mathcal{A}} V_{x}^{( \pm 1)}$. Hence, we conclude Corollary 5.4.

\subsubsection{Completion of proof of Theorem 5.5}

Proposition 5.14 For all $x \in \Lambda, \beta \geq 0$ and $\varepsilon \in\{ \pm 1\}$, we have

$$
\left(n_{x} \otimes \mathbb{1}+\mathbb{1} \otimes n_{x}\right)\left(n_{x} \otimes \mathbb{1}-\mathbb{1} \otimes n_{x}\right) \succeq 0 \quad \text { w.r.t. } \mathscr{L}^{2}(\mathfrak{X})_{+} .
$$

Proof. Note that since $\vartheta \nu_{x}^{*} \vartheta=-\nu_{x}$, we have

$$
n_{x} \otimes \mathbb{1}+\mathbb{1} \otimes n_{x}=\mathbb{1} \otimes \nu_{x}=-\mathcal{R}\left(\nu_{x}\right),
$$

and

$$
n_{x} \otimes \mathbb{1}-\mathbb{1} \otimes n_{x}=-\nu_{x} \otimes \mathbb{1}=-\mathcal{L}\left(\nu_{x}\right) .
$$

Thus, we have $\left(n_{x} \otimes \mathbb{1}+\mathbb{1} \otimes n_{x}\right)\left(n_{x} \otimes \mathbb{1}-\mathbb{1} \otimes n_{x}\right)=\mathcal{L}\left(\nu_{x}\right) \mathcal{R}\left(\nu_{x}\right) \succeq 0$ w.r.t. $\mathscr{L}^{2}(\mathfrak{X})_{+}$. 
By Proposition 5.14, we see that

$$
\begin{aligned}
& \prod_{j=1}^{n}\left[n_{x_{j}}\left(s_{j}\right) \otimes \mathbb{1}+\mathbb{1} \otimes n_{x_{j}}\left(s_{j}\right)\right]\left[n_{x_{j}}\left(s_{j}\right) \otimes \mathbb{1}-\mathbb{1} \otimes n_{x_{j}}\left(s_{j}\right)\right] \mathrm{e}^{-\beta H_{\mathrm{ext}}} \\
= & \underbrace{\mathrm{e}^{-s H_{\mathrm{ext}}}}_{\succeq 0} \underbrace{\left[n_{x_{1}} \otimes \mathbb{1}+\mathbb{1} \otimes n_{x_{1}}\right]\left[n_{x_{1}} \otimes \mathbb{1}-\mathbb{1} \otimes n_{x_{1}}\right]}_{\succeq 0} \underbrace{\mathrm{e}^{-\left(s_{2}-s_{1}\right) H_{\mathrm{ext}}}}_{\succeq 0} \times \cdots \\
& \cdots \times \underbrace{\left[n_{x_{n}} \otimes \mathbb{1}+\mathbb{1} \otimes n_{x_{n}}\right]\left[n_{x_{n}} \otimes \mathbb{1}-\mathbb{1} \otimes n_{x_{n}}\right]}_{\succeq 0} \underbrace{\mathrm{e}^{-\left(\beta-s_{n}\right) H_{\text {ext }}}}_{\succeq 0} \succeq 0 \quad \text { w.r.t. } \mathscr{L}^{2}(\mathfrak{X})_{+} .
\end{aligned}
$$

Therefore, Theorem 5.5 follows from Proposition 3.9 ,

\subsubsection{Completion of proof of Theorem $\underline{5.6}$}

By Propositions 5.13 and $\left[5.14\right.$, we know $\left[\prod_{j=1}^{n} \alpha_{x_{j}}^{\left(\mu_{j}\right)}\left(s_{j}\right)\right] \mathrm{e}^{-\beta H_{\text {ext }}} \succeq 0$ w.r.t. $\mathscr{L}^{2}(\mathfrak{X})_{+}$. Thus, Theorem 5.6 immediately follows from Proposition 3.9 ,

\subsection{Proof of Example 5}

The proof of Example 5 is similar to that of Example 4, so we only provide a sketch. By the Duhamel formula (4.55), we obtain

$$
\begin{aligned}
& \frac{\partial}{\partial t_{x y}}\left\langle C^{A}\right\rangle_{\beta} \\
= & \sum_{n \geq 1} \frac{n\left(t_{x y}\right)^{n-1}}{2} \int_{0 \leq t_{1} \leq \cdots \leq t_{n} \leq \beta}\left\langle\left\langle\left(C^{A} \otimes \mathbb{1}-\mathbb{1} \otimes C^{A}\right)\left(\mathrm{K}_{n} \otimes \mathbb{1}-\mathbb{1} \otimes \mathrm{K}_{n}\right)\right\rangle\right\rangle_{H_{\mathrm{ext}}^{\prime}, \beta} d t_{1} \cdots d t_{n},
\end{aligned}
$$

where $\mathrm{K}_{n}=\mathrm{e}^{-t_{1} H^{\prime}} \cos \left(\theta_{x}-\theta_{y}\right) \mathrm{e}^{t_{1} H^{\prime}} \cdots \mathrm{e}^{-t_{n} H^{\prime}} \cos \left(\theta_{x}-\theta_{y}\right) \mathrm{e}^{t_{n} H^{\prime}}$ with $H^{\prime}=H+$ $t_{x y} \cos \left(\theta_{x}-\theta_{y}\right)$ and $H_{\text {ext }}^{\prime}=H^{\prime} \otimes \mathbb{1}+\mathbb{1} \otimes H^{\prime}$. Since $\mathrm{e}^{-t H_{\text {ext }}^{\prime}} \succeq 0, C^{A} \otimes \mathbb{1}-\mathbb{1} \otimes C^{A} \succeq 0$ and $\mathrm{K}_{n} \otimes \mathbb{1}-\mathbb{1} \otimes \mathrm{K}_{n} \succeq 0$ w.r.t. $\mathscr{L}^{2}(\mathfrak{X})_{+}$, we know that the RHS of (5.47) is positive. Thus, we obtain (i). Similarly, we have

$$
\begin{aligned}
& \frac{\partial}{\partial t_{x y}}\left\langle n_{z}^{2}\right\rangle_{\beta} \\
& =\sum_{n \geq 1} \frac{n\left(t_{x y}\right)^{n-1}}{2} \int_{0 \leq t_{1} \leq \cdots \leq t_{n} \leq \beta}\left\langle\langle\underbrace{\left(n_{z}^{2} \otimes \mathbb{1}-\mathbb{1} \otimes n_{z}^{2}\right)}_{\succeq 0 \text { by Proposition [5.14 }}\left(\mathrm{K}_{n} \otimes \mathbb{1}-\mathbb{1} \otimes \mathrm{K}_{n}\right)\rangle\right\rangle_{H_{\text {ext }}^{\prime}, \beta} d t_{1} \cdots d t_{n} \\
& \geq 0 \text {. }
\end{aligned}
$$

Hence, we arrive at (ii). 
(iii) Let $H^{\prime \prime}=H-\frac{U_{x}}{2} n_{x}^{2}$. By Proposition 5.14, Corollary 5.12, and the Duhamel formula (4.55), we obtain

$$
\begin{aligned}
& \left.\frac{\partial}{\partial U_{x}}\left\langle C^{A}\right\rangle_{\beta}\right|_{U_{x}=0} \\
& =-\frac{\beta}{2} \int_{0}^{\beta}\left\langle\langle\underbrace{\left(C^{A} \otimes \mathbb{1}-\mathbb{1} \otimes C^{A}\right)}_{\succeq 0} \underbrace{e^{-t H_{\text {ext }}^{\prime \prime}}}_{\succeq 0} \underbrace{\left(n_{x}^{2} \otimes \mathbb{1}-\mathbb{1} \otimes n_{x}^{2}\right)}_{\succeq 0} \mathrm{e}^{t H_{\mathrm{ext}}^{\prime \prime}}\rangle\right\rangle_{H_{\mathrm{ext}}^{\prime \prime}, \beta} d t \\
& \leq 0 \text {, }
\end{aligned}
$$

where $H_{\text {ext }}^{\prime \prime}=H^{\prime \prime} \otimes \mathbb{1}+\mathbb{1} \otimes H^{\prime \prime}$. This completes the proof.

\section{Bose-Hubbard model}

\subsection{Results}

Let $\Lambda$ be a finite subset of $\mathbb{R}^{d}$. The Bose-Hubbard model on $\Lambda$ is defined by

$$
H=\sum_{x, y \in \Lambda}\left(-t_{x y}\right) a_{x}^{*} a_{y}+\sum_{x \in \Lambda} U_{x} n_{x}\left(n_{x}-\mathbb{1}\right)-\sum_{x \in \Lambda} \lambda_{x}\left(a_{x}^{*}+a_{x}\right)-\mu N_{\mathrm{b}} .
$$

$H$ acts in the bosonic Fock space $\mathfrak{B}=\oplus_{n=0}^{\infty} \otimes_{\mathrm{s}}^{n} \ell^{2}(\Lambda)$, where $\otimes_{\mathrm{s}}^{n} \ell^{2}(\Lambda)$ is the $n$-fold symmetric tensor product of $\ell^{2}(\Lambda)$ with $\otimes_{\mathrm{s}}^{0} \ell^{2}(\Lambda)=\mathbb{C}$. $a_{x}$ is the bosonic annihilation operator satisfying the canonical commutation relations (CCRs):

$$
\left[a_{x}, a_{y}^{*}\right]=\delta_{x y}, \quad\left[a_{x}, a_{y}\right]=0 .
$$

$n_{x}=a_{x}^{*} a_{x}$ is the number operator at site $x \in \Lambda$ and $N_{\mathrm{b}}=\sum_{x \in \Lambda} n_{x}$ is the total number operator.

We assume the following:

(A. 1) $t_{x y} \geq 0, \quad U_{x}>0, \quad \lambda_{x} \geq 0$ for all $x, y \in \Lambda$.

(A. 2) $t_{x y}=t_{y x}$ for all $x, y \in \Lambda$ and $t_{x x}=0$ for all $x \in \Lambda$.

(A. 3) $\mu \in \mathbb{R}$.

Under these conditions, we see that $\mathrm{e}^{-\beta H}$ is in the trace class for all $\beta>0$. The thermal expectation value is defined as

$$
\langle X\rangle_{\beta}=\operatorname{Tr}\left[X \mathrm{e}^{-\beta H}\right] / Z_{\beta}, \quad Z_{\beta}=\operatorname{Tr}\left[\mathrm{e}^{-\beta H}\right] .
$$

For each densely defined linear operator $X, X^{\#}(\#=+$ or -$)$ means

$$
X^{\#}= \begin{cases}X & \text { if } \#=- \\ X^{*} & \text { if } \#=+.\end{cases}
$$

Set $\mathbb{N}_{0}=\{0\} \cup \mathbb{N}$. For each $\mathbf{m}=\left\{m_{x}\right\}_{x \in \Lambda} \in \mathbb{N}_{0}^{\Lambda}$ and $\#=\left\{\#_{x}\right\}_{x \in \Lambda} \in\{ \pm\}^{\Lambda}$, define

$$
I(\mathbf{m} ; \#)=\prod_{x \in \Lambda}\left(a_{x}^{\# x}\right)^{m_{x}}
$$


with $\left(a_{x}^{\# x}\right)^{0}=\mathbb{1}$. Now we define

$$
\mathfrak{A}=\operatorname{Coni}\left\{I(\mathbf{m} ; \#) \mid \mathbf{m} \in \mathbb{N}_{0}^{\Lambda}, \# \in\{ \pm\}^{\Lambda}\right\} .
$$

Note that for all $A \in \mathfrak{A}, A \mathrm{e}^{-\beta H}$ is in the trace class for all $\beta>0$. Thus, $\langle A\rangle_{\beta}$ is finite.

Theorem 6.1 (First Griffiths inequality) Let $A_{1}, \ldots, A_{n} \in \mathfrak{A}$. For all $0 \leq s_{1} \leq$ $s_{2} \leq \cdots \leq s_{n}<\beta$, we have

$$
\left\langle\prod_{j=1}^{\stackrel{n}{\longrightarrow}} A_{j}\left(s_{j}\right)\right\rangle_{\beta} \geq 0
$$

where $A(s)=\mathrm{e}^{-s H} A \mathrm{e}^{s H}$.

To state the second quantum Griffiths inequality, we introduce the following notation:

$$
\langle\langle Y\rangle\rangle_{\beta}:=\operatorname{Tr}_{\mathfrak{B} \otimes \mathfrak{B}}\left[Y \mathrm{e}^{-\beta H_{\mathrm{ext}}}\right] / Z_{\beta}^{2}, \quad H_{\mathrm{ext}}=H \otimes \mathbb{1}+\mathbb{1} \otimes H .
$$

Theorem 6.2 Let $x_{1}, \ldots, x_{n}, y_{1}, \ldots, y_{n} \in \Lambda$. For each $0 \leq s_{1} \leq t_{1} \leq s_{2} \leq t_{2} \leq \cdots \leq$ $s_{n} \leq t_{n}<\beta, \#_{1}, \ldots, \#_{n} \in\{ \pm\}$ and $\varepsilon_{1}, \ldots, \varepsilon_{n} \in\{ \pm 1\}$, we have

$$
\left\langle\left\langle\prod_{j=1}^{n}\left[a_{x_{j}}^{\#_{j}}\left(s_{j}\right) \otimes \mathbb{1}+\varepsilon_{j} \mathbb{1} \otimes a_{x_{j}}^{\#_{j}}\left(s_{j}\right)\right]\left[a_{y_{j}}^{\#_{j}}\left(t_{j}\right) \otimes \mathbb{1}+\varepsilon_{j} \mathbb{1} \otimes a_{y_{j}}^{\#_{j}}\left(t_{j}\right)\right]\right\rangle\right\rangle_{\beta} \geq 0
$$

where $\overline{\#}=-\# 12$ and $a_{x}^{\#}(s)=\mathrm{e}^{-s H} a_{x}^{\#} \mathrm{e}^{s H}$.

Example 6 Consider the case where $n=1, \varepsilon_{1}=-1$, and $\#_{1}=+$. Then we have

$$
\left\langle a_{x}^{*}(s) a_{y}(t)\right\rangle_{\beta}-\left\langle a_{x}^{*}\right\rangle_{\beta}\left\langle a_{y}\right\rangle_{\beta} \geq 0
$$

for all $x, y \in \Lambda$ and $0 \leq s \leq t<\beta$. From this, we have

$$
\left(a_{x}^{*}, a_{y}\right)_{\beta}-\left\langle a_{x}^{*}\right\rangle_{\beta}\left\langle a_{y}\right\rangle_{\beta} \geq 0 .
$$

In addition, by Theorem 6.1, it follows that

$$
\left(a_{x}^{*}, a_{y}\right)_{\beta} \geq 0, \quad\left\langle a_{x}^{*}\right\rangle_{\beta} \geq 0,\left\langle a_{y}\right\rangle_{\beta} \geq 0 . \quad \diamond
$$

We can generalize Theorem 6.2. To state our result, we need to introduce the following:

$$
\begin{aligned}
& \alpha_{+1, x}=a_{x} \otimes \mathbb{1}+\mathbb{1} \otimes a_{x}, \\
& \alpha_{-1, x}=-i\left(a_{x} \otimes \mathbb{1}-\mathbb{1} \otimes a_{x}\right),
\end{aligned}
$$

where $i=\sqrt{-1}$.

\footnotetext{
${ }^{12}$ To be precise, $\bar{\mp}=-$ and $\overline{=}=+$.
} 
Theorem 6.3 (Second Griffiths inequality) Let $x_{1}, \ldots, x_{n} \in \Lambda$. For all $\#_{1}, \ldots, \#_{n} \in$ $\{ \pm\}, \varepsilon_{1}, \ldots, \varepsilon_{n} \in\{ \pm 1\}$ and $0 \leq s_{1} \leq s_{2} \leq \cdots \leq s_{n}<\beta$, we have

$$
\left\langle\left\langle\prod_{j=1}^{n} \alpha_{\varepsilon_{j}, x_{j}}^{\#_{j}}\left(s_{j}\right)\right\rangle\right\rangle_{\beta} \geq 0,
$$

where $\alpha_{\varepsilon, x}^{\#}(s)=\mathrm{e}^{-s H_{\mathrm{ext}}} \alpha_{\varepsilon, x}^{\#} \mathrm{e}^{s H_{\mathrm{ext}}}$.

Remark 6.4 If $\lambda_{x}>0$ for all $x \in \Lambda$, then we can prove that the ground state of $H$ is unique 13 . In this case, our results are valid at $\beta=\infty$. $\diamond$

Example 7 Consider the case where $n=3, \#_{1}=+, \#_{2}=\#_{3}=-$, and $\varepsilon_{1} \varepsilon_{2} \varepsilon_{3}=1$. We have

$$
\left\langle a_{1}^{*} a_{2} a_{3}\right\rangle-\left\langle a_{1}^{*}\right\rangle\left\langle a_{2} a_{3}\right\rangle-\left\langle a_{2}\right\rangle\left\langle a_{1}^{*} a_{3}\right\rangle+\left\langle a_{3}\right\rangle\left\langle a_{1}^{*} a_{2}\right\rangle \geq 0
$$

for $\left(\varepsilon_{1}, \varepsilon_{2}, \varepsilon_{3}\right)=(-1,-1,+1)$, and

$$
\left\langle a_{1}^{*} a_{2} a_{3}\right\rangle-\left\langle a_{1}^{*}\right\rangle\left\langle a_{2} a_{3}\right\rangle+\left\langle a_{2}\right\rangle\left\langle a_{1}^{*} a_{3}\right\rangle-\left\langle a_{3}\right\rangle\left\langle a_{1}^{*} a_{2}\right\rangle \geq 0
$$

for $\left(\varepsilon_{1}, \varepsilon_{2}, \varepsilon_{3}\right)=(-1,+1,-1)$, where we use the abbreviation $a_{j}^{\#}=a_{x_{j}}^{\#}\left(s_{j}\right)$. On the other hand, we have

$$
\left\langle a_{1}^{*} a_{2} a_{3}\right\rangle+\left\langle a_{1}^{*}\right\rangle\left\langle a_{2} a_{3}\right\rangle-\left\langle a_{2}\right\rangle\left\langle a_{1}^{*} a_{3}\right\rangle-\left\langle a_{3}\right\rangle\left\langle a_{1}^{*} a_{2}\right\rangle \leq 0
$$

for $\left(\varepsilon_{1}, \varepsilon_{2}, \varepsilon_{3}\right)=(+1,-1,-1)$. Combining (6.16) and (6.17), we get

$$
\left\langle a_{1}^{*} a_{2} a_{3}\right\rangle-\left\langle a_{1}^{*}\right\rangle\left\langle a_{2} a_{3}\right\rangle \geq 0 . \quad \diamond
$$

If $U_{x} \equiv 0$, then we obtain a stronger result as follows.

Theorem 6.5 Assume that $U_{x}=0$ for all $x \in \Lambda$. Assume that the matrix $\left(-t_{x y}-\right.$ $\left.\mu \delta_{x y}\right)_{x, y}$ is positive-definite 14 Let $x_{1}, \ldots, x_{n} \in \Lambda$. For all $\#_{1}, \ldots, \#_{n} \in\{ \pm\}, \varepsilon_{1}, \ldots, \varepsilon_{n} \in$ $\{ \pm 1\}$ and $0 \leq s_{1} \leq \cdots \leq s_{n}<\beta$, we have

$$
\left\langle\left\langle\prod_{j=1}^{n}\left[a_{x_{j}}^{\#_{j}}\left(s_{j}\right) \otimes \mathbb{1}+\varepsilon_{j} \mathbb{1} \otimes a_{x_{j}}^{\#_{j}}\left(s_{j}\right)\right]\right\rangle\right\rangle_{\beta} \geq 0 .
$$

Corollary 6.6 Under the same assumptions as Theorem 6.5, we have

$$
\left\langle A_{1} A_{2}\right\rangle_{\beta}-\left\langle A_{1}\right\rangle_{\beta}\left\langle A_{2}\right\rangle_{\beta} \geq 0
$$

for all $A_{1}, A_{2} \in \mathfrak{A}$.

Example 8 Let $A \in \mathfrak{A}$. Under the same assumptions as in Theorem 6.5, we have the following:

(i) $\langle A\rangle_{\beta}$ is monotonically increasing in $t_{x y}$.

(ii) $\langle A\rangle_{\beta}$ is monotonically increasing in $\lambda_{x}$.

The proofs of these properties are similar to those of Examples 4 and 5 . $\diamond$

\footnotetext{
${ }^{13}$ This fact follows from an application of the Perron-Frobenius-Faris theorem 12 .

${ }^{14}$ This assumption is needed in order to guarantee that $\mathrm{e}^{-\beta H}$ is a trace class operator.
} 


\subsection{Proof of Theorem 6.1}

In this section, we will often discuss unbounded operators. Thus, we have to extend definitions of our operator inequalities as follows:

Definition 6.7 Let $A$ be a densely defined linear operator in $\mathfrak{H}$. If $A x \geq 0$ w.r.t. $\mathfrak{P}$ for all $x \in \mathfrak{P} \cap \operatorname{dom}(A)$, then we also write $A \unrhd 0$ w.r.t. $\mathfrak{P}$. Note that

$$
\langle x \mid A y\rangle \geq 0 \quad \text { for all } x \in \mathfrak{P} \text { and } y \in \mathfrak{P} \cap \operatorname{dom}(A) .
$$

For each $\mathbf{N}=\left\{N_{x}\right\}_{x \in \Lambda} \in \mathbb{N}_{0}^{\Lambda}$, we set

$$
|\mathbf{N}\rangle=\left(\prod_{x \in \Lambda} N_{x} !\right)^{-1 / 2} \prod_{x \in \Lambda}\left(a_{x}^{*}\right)^{N_{x}} \Omega,
$$

where $\Omega$ is the Fock vacuum. Then $\left\{|\mathbf{N}\rangle \mid \mathbf{N} \in \mathbb{N}_{0}^{\Lambda}\right\}$ is a CONS of $\mathfrak{B}$.

Definition 6.8 A standard self-dual cone in $\mathfrak{B}$ is defined by

$$
\left.\mathfrak{B}_{+}=\left\{\psi \in \mathfrak{B}\left|\psi=\sum_{\mathbf{N} \in \mathbb{N}_{0}^{\Lambda}} \psi_{\mathbf{N}}\right| \mathbf{N}\right\rangle, \quad \psi_{\mathbf{N}} \geq 0 \forall \mathbf{N} \in \mathbb{N}_{0}^{\Lambda}\right\} .
$$

$\mathfrak{B}_{+}$was introduced by Fröhlich [16], see also [46]. $\diamond$

Remark 6.9 $|\mathbf{N}\rangle \in \mathfrak{B}_{+}$for all $\mathbf{N} \in \mathbb{N}_{0}^{\Lambda} . \diamond$

The following lemma is useful in this section.

Lemma 6.10 Let $A$ be a densely defined linear operator on $\mathfrak{B}$. Let $P_{\ell}$ be the orthogonal projection onto $\oplus_{n=0}^{\ell} \otimes_{\mathrm{s}}^{n} \ell^{2}(\Lambda)$. Assume the following:

(i) $|\mathbf{N}\rangle \in \operatorname{dom}(A)$ for all $\mathbf{N} \in \mathbb{N}_{0}^{\Lambda}$.

(ii) $A P_{\ell} \varphi \rightarrow A \varphi$ as $\ell \rightarrow \infty$ for all $\varphi \in \operatorname{dom}(A)$.

Then the following are equivalent.

(a) $A \unrhd 0$ w.r.t. $\mathfrak{B}_{+}$.

(b) $\langle\mathbf{M}|A| \mathbf{N}\rangle \geq 0$ for all $\mathbf{M}, \mathbf{N} \in \mathbb{N}_{0}^{\Lambda} \sqrt{15}$.

Proof. (a) $\Longrightarrow(\mathrm{b})$ : This is immediate.

(b) $\Longrightarrow$ (a): Let $A_{\ell}=P_{\ell} A P_{\ell}$. Then, for all $\varphi \in \mathfrak{B}_{+}$and $\psi \in \operatorname{dom}(A) \cap \mathfrak{B}_{+}$, we see that

$$
\left\langle\varphi \mid A_{\ell} \psi\right\rangle=\sum_{|\mathbf{M}| \leq \ell,|\mathbf{N}| \leq \ell} \underbrace{\varphi_{\mathbf{M}}}_{\geq 0} \underbrace{\psi_{\mathbf{N}}}_{\geq 0} \underbrace{\langle\mathbf{M}|A| \mathbf{N}\rangle}_{\geq 0} \geq 0,
$$

where $|\mathbf{N}|=\sum_{x \in \Lambda} N_{x}$. Taking $\ell \rightarrow \infty$, we obtain $\langle\varphi \mid A \psi\rangle \geq 0$, which implies $A \psi \geq 0$ w.r.t. $\mathfrak{B}_{+}$.

\footnotetext{
${ }^{15}\langle\psi|X| \phi\rangle:=\langle\psi \mid X \phi\rangle$.
} 
Proposition 6.11 We have $a_{x} \unrhd 0, a_{x}^{*} \unrhd 0$ w.r.t. $\mathfrak{B}_{+}$for all $x \in \Lambda$.

Proof. It is not difficult to verify that $a_{x}$ and $a_{x}^{*}$ satisfy the assumptions of Lemma6.10. Moreover, we see that $\left\langle\mathbf{M}\left|a_{x}\right| \mathbf{N}\right\rangle \geq 0$ and $\left\langle\mathbf{M}\left|a_{x}^{*}\right| \mathbf{N}\right\rangle \geq 0$ for all $\mathbf{M}, \mathbf{N} \in \mathbb{N}_{0}^{\Lambda}$. Thus, we obtain the desired assertion by Lemma 6.10,

Corollary 6.12 For all $A \in \mathfrak{A}$, it holds that $A \unrhd 0$ w.r.t. $\mathfrak{B}_{+}$.

Proposition 6.13 We have $\mathrm{e}^{-\beta H} \unrhd 0$ w.r.t. $\mathfrak{B}_{+}$for all $\beta \geq 0$.

Proof. Let $P_{\ell}$ be the orthogonal projection defined in Lemma 6.10, Let $H_{\ell}=P_{\ell} H P_{\ell}$. Since $H_{\ell}$ converges to $H$ in the strong resolvent sense as $\ell \rightarrow \infty$, it suffices to show that

$$
\mathrm{e}^{-\beta H_{\ell}} \unrhd 0 \quad \text { w.r.t. } \mathfrak{B}_{+} \text {for all } \beta \geq 0 \text { and } \ell \in \mathbb{N} .
$$

To this end, we set

$$
T=\sum_{x, y \in \Lambda} t_{x y} a_{x}^{*} a_{y}+\sum_{x \in \Lambda} \lambda_{x}\left(a_{x}+a_{x}^{*}\right)+\mu N_{\mathrm{b}}, \quad U=\sum_{x \in \Lambda} U_{x} n_{x}\left(n_{x}-\mathbb{1}\right) .
$$

Let $T_{\ell}=P_{\ell} T P_{\ell}, U_{\ell}=P_{\ell} U P_{\ell}$. Then $T_{\ell}$ and $U_{\ell}$ are bounded for each $\ell \in \mathbb{N}$. We observe that $\left\langle\mathbf{M}\left|T_{\ell}\right| \mathbf{N}\right\rangle \geq 0$. Thus, by Proposition $\underline{\text { A.2. }}, T_{\ell} \unrhd 0$ w.r.t. $\mathfrak{B}_{+}$holds for all $\ell \in \mathbb{N}$. On the other hand,

$$
\left\langle\mathbf{M}\left|\mathrm{e}^{-\beta U_{\ell}}\right| \mathbf{N}\right\rangle=\left\{\begin{array}{ll}
\exp \left\{-\beta \sum_{x \in \Lambda} N_{x}\left(N_{x}-1\right)\right\} \delta_{\mathbf{M N}} & \text { if }|\mathbf{M}| \leq \ell \text { and }|\mathbf{N}| \leq \ell \\
\delta_{\mathbf{M N}} & \text { if }|\mathbf{M}|>\ell \text { or }|\mathbf{N}|>\ell
\end{array} .\right.
$$

This means $\left\langle\mathbf{M}\left|\mathrm{e}^{-\beta U_{\ell}}\right| \mathbf{N}\right\rangle \geq 0$. Thus, applying Proposition A.2, we conclude $\mathrm{e}^{-\beta U_{\ell}} \unrhd 0$ w.r.t. $\mathfrak{B}_{+}$for all $\beta \geq 0$ and $\ell \in \mathbb{N}$. Hence, by Proposition A.5. we conclude $\mathrm{e}^{-\beta H_{\ell}} \unrhd 0$ w.r.t. $\mathfrak{B}_{+}$for all $\beta \geq 0$ and $\ell \in \mathbb{N}$.

Corollary 6.14 Let $x_{1}, \ldots, x_{n} \in \Lambda$. For all $\#_{1}, \ldots, \#_{n} \in\{ \pm\}$ and $0 \leq s_{1} \leq s_{2} \leq$ $\cdots \leq s_{n}<\beta$, we have

$$
\mathrm{e}^{-s_{1} H} a_{x_{1}}^{\# 1} \mathrm{e}^{-\left(s_{2}-s_{1}\right) H} a_{x_{2}}^{\# 2} \mathrm{e}^{-\left(s_{3}-s_{2}\right) H} \cdots \mathrm{e}^{-\left(s_{n}-s_{n-1}\right) H} a_{x_{n}}^{\# n} \mathrm{e}^{-\left(\beta-s_{n}\right) H} \unrhd 0
$$

w.r.t. $\mathfrak{B}_{+}$.

\subsubsection{Completion of proof of Theorem 6.1}

By Corollary 6.12 and Proposition 6.13, we have

$$
\begin{aligned}
& {\left[\prod_{j=1}^{n} A_{j}\left(s_{j}\right)\right] \mathrm{e}^{-\beta H} } \\
= & \underbrace{\mathrm{e}^{-s H}}_{\unrhd 0} \underbrace{A_{1}}_{\unrhd 0} \underbrace{e^{-\left(s_{2}-s_{1}\right) H}}_{\unrhd 0} \cdots \underbrace{A_{n}}_{\unrhd 0} \underbrace{e^{-\left(\beta-s_{n}\right) H}}_{\unrhd 0} \unrhd 0 \quad \text { w.r.t. } \mathfrak{B}_{+} .
\end{aligned}
$$

Thus, by Proposition A.1, we conclude Theorem 6.1. 


\subsection{Proof of Theorems 6.2 and 6.3}

Let $\mathfrak{B}_{\text {ext }}=\mathfrak{B} \otimes \mathfrak{B}$. We introduce a new representation of the CCRs as follows. Let

$$
\xi_{x}=\frac{1}{\sqrt{2}}\left(a_{x} \otimes \mathbb{1}+\mathbb{1} \otimes a_{x}\right), \quad \eta_{x}=-\frac{1}{\sqrt{2}}\left(a_{x} \otimes \mathbb{1}-\mathbb{1} \otimes a_{x}\right) .
$$

$\xi_{x}$ and $\eta_{x}$ act in $\mathfrak{B}_{\text {ext }}$ and are closable. We denote their closures by the same symbols. Then $\left\{\xi_{x}, \eta_{x}\right\}$ satisfies the following CCRs:

$$
\begin{aligned}
& {\left[\xi_{x}, \xi_{y}\right]=0, \quad\left[\eta_{x}, \eta_{y}\right]=0, \quad\left[\xi_{x}, \eta_{y}\right]=0,} \\
& {\left[\xi_{x}, \xi_{y}^{*}\right]=\delta_{x y}, \quad\left[\eta_{x}, \eta_{y}^{*}\right]=\delta_{x y},\left[\xi_{x}, \eta_{y}^{*}\right]=0 .}
\end{aligned}
$$

Using $\xi_{x}$ and $\eta_{x}$, we can rewrite $H$ as

$$
H_{\mathrm{ext}}=-\mathbb{T}+\mathbb{U}
$$

where

$$
\mathbb{T}=\sum_{x, y \in \Lambda} t_{x y}\left(\xi_{x}^{*} \xi_{y}+\eta_{x}^{*} \eta_{y}\right)+\sqrt{2} \sum_{x \in \Lambda} \lambda_{x}\left(\xi_{x}+\xi_{x}^{*}\right)
$$

and

$$
\begin{aligned}
\mathbb{U}= & \mathbb{U}_{d}+\mathbb{U}_{o}, \\
\mathbb{U}_{d}= & \sum_{x \in \Lambda} \frac{U_{x}}{2}\left(\xi_{x}^{*} \xi_{x} \xi_{x}^{*} \xi_{x}+4 \xi_{x}^{*} \xi_{x} \eta_{x}^{*} \eta_{x}+\eta_{x}^{*} \eta_{x} \eta_{x}^{*} \eta_{x}\right) \\
& -\sum_{x \in \Lambda}\left(\frac{1}{2} U_{x}+\mu\right)\left(\xi_{x}^{*} \xi_{x}+\eta_{x}^{*} \eta_{x}\right), \\
\mathbb{U}_{o}= & \sum_{x \in \Lambda} \frac{U_{x}}{2}\left(\xi_{x}^{*} \xi_{x}^{*} \eta_{x} \eta_{x}+\xi_{x} \xi_{x} \eta_{x}^{*} \eta_{x}^{*}\right) .
\end{aligned}
$$

Let $\Omega_{\text {ext }}=\Omega \otimes \Omega \in \mathfrak{B}_{\text {ext }}$. For each $\mathbf{M}, \mathbf{N} \in \mathbb{N}_{0}^{\Lambda}$, we define

$$
|\mathbf{M}, \mathbf{N}\rangle\rangle=\left(\prod_{x \in \Lambda} M_{x} ! N_{x} !\right)^{-1 / 2} \prod_{x \in \Lambda}\left(\xi_{x}^{*}\right)^{M_{x}}\left(\eta_{x}^{*}\right)^{N_{x}} \Omega_{\mathrm{ext}} .
$$

Clearly, $\left.\{|\mathbf{M}, \mathbf{N}\rangle\rangle \mid \mathbf{M}, \mathbf{N} \in \mathbb{N}_{0}^{\Lambda}\right\}$ is a CONS of $\mathfrak{B}_{\text {ext }}$.

Definition 6.15 We define a self-dual cone in $\mathfrak{B}_{\text {ext }}$ by

$$
\left.\left.\mathfrak{B}_{\text {ext },+}=\left\{\Psi \in \mathfrak{B}_{\text {ext }}\left|\Psi=\sum_{\mathbf{M}, \mathbf{N} \in \mathbb{N}_{0}^{\Lambda}} \Psi_{\mathbf{M}, \mathbf{N}}\right| \mathbf{M}, \mathbf{N}\right\rangle\right\rangle, \Psi_{\mathbf{M}, \mathbf{N}} \geq 0 \forall \mathbf{M}, \mathbf{N} \in \mathbb{N}_{0}^{\Lambda}\right\} .
$$

We can prove the following in a manner similar to that used for Proposition 6.11.

Proposition 6.16 We have $\xi_{x}^{\#} \unrhd 0, \eta_{x}^{\#} \unrhd 0$ w.r.t. $\mathfrak{B}_{\mathrm{ext},+}$ for all $x \in \Lambda$ and $\# \in\{ \pm\}$. 
Proposition 6.17 Let

$$
\mathscr{U}=\exp \left\{-i \frac{\pi}{2} \sum_{x \in \Lambda} \eta_{x}^{*} \eta_{x}\right\} .
$$

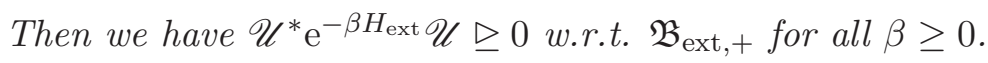

Proof. Let $\hat{H}_{\text {ext }}=\mathscr{U}^{*} H_{\text {ext }} \mathscr{U}$. It is important to note that

$$
\mathscr{U}^{*} \mathbb{U}_{o} \mathscr{U}=-\mathbb{U}_{o} .
$$

Thus, we have

$$
\hat{H}_{\mathrm{ext}}=-\mathbb{K}+\mathbb{U}_{d}
$$

where $\mathbb{K}=\mathbb{T}+\mathbb{U}_{o}$. Let $\mathcal{P}_{\ell}$ be the orthogonal projection onto the closed subspace spanned by $\left.\{|\mathbf{M}, \mathbf{N}\rangle\rangle\left|\mathbf{M}, \mathbf{N} \in \mathbb{N}_{0}^{\Lambda},\right| \mathbf{M}|+| \mathbf{N} \mid \leq \ell\right\}$. Let $\hat{H}_{\text {ext }, \ell}=\mathcal{P}_{\ell} \hat{H}_{\text {ext }} \mathcal{P}_{\ell}$. Since $\hat{H}_{\text {ext }, \ell}$ converges to $\hat{H}_{\text {ext }}$ in the strong resolvent sense as $\ell \rightarrow \infty$, it suffices to show that

$$
\exp \left(-\beta \hat{H}_{\text {ext }, \ell}\right) \unrhd 0 \quad \text { w.r.t. } \mathfrak{B}_{\text {ext },+} \text { for all } \beta \geq 0 \text { and } \ell \in \mathbb{N} .
$$

The proof of this is almost parallel to that of Proposition 6.13. For reader's convenience, we provide a sketch of it. Let $\mathbb{K}_{\ell}=\mathcal{P}_{\ell} \mathbb{K} \mathcal{P}_{\ell}$ and $\mathbb{U}_{d, \ell}=\mathcal{P}_{\ell} \mathbb{U}_{d} \mathcal{P}_{\ell}$. First, we show that $\mathbb{K}_{\ell} \unrhd 0$ w.r.t. $\mathfrak{B}_{\text {ext, }+}$ for all $\ell \in \mathbb{N}$. Next we show that $\exp \left(-\beta \mathbb{U}_{d, \ell}\right) \unrhd 0$ w.r.t. $\mathfrak{B}_{\text {ext, }+}$ for all $\beta \geq 0$ and $\ell \in \mathbb{N}$. Then by Proposition A.5, we conclude (6.43).

Proposition 6.18 Set $\hat{\alpha}_{\varepsilon, x}^{\#}=\mathscr{U}^{*} \alpha_{\varepsilon, x}^{\#} \mathscr{U}$. Then we have $\hat{\alpha}_{\varepsilon, x}^{\#} \unrhd 0$ w.r.t. $\mathfrak{B}_{\text {ext },+ \text { for all }}$ $x \in \Lambda, \varepsilon \in\{ \pm 1\}$ and $\# \in\{-,+\}$.

Proof. By Proposition 6.16, we have

$$
\begin{array}{ll}
\hat{\alpha}_{+1 . x}^{\#}=\sqrt{2} \xi_{x}^{\#} \unrhd 0 & \text { w.r.t. } \mathfrak{B}_{\text {ext },+}, \\
\hat{\alpha}_{-1 . x}^{\#}=\sqrt{2} \eta_{x}^{\#} \unrhd 0 & \text { w.r.t. } \mathfrak{B}_{\text {ext },+}^{\#}
\end{array}
$$

\subsubsection{Completion of proofs of Theorems 6.2 and 6.3}

We only prove Theorem 6.3, since Theorem 6.2 is a corollary of it. Let $\hat{H}_{\text {ext }}=$ $\mathscr{U}^{*} H_{\text {ext }} \mathscr{U}$. Then we have

$$
\begin{aligned}
& \mathscr{U}^{*} \prod_{j=1}^{\vec{n}} \alpha_{\varepsilon_{j}, x_{j}}^{\#_{j}}\left(s_{j}\right) \mathscr{U} \mathrm{e}^{\beta \hat{H}_{\mathrm{ext}}} \\
= & \underbrace{\mathrm{e}^{-s_{1} \hat{H}_{\mathrm{ext}}}}_{\unrhd 0} \underbrace{\hat{\alpha}_{\varepsilon_{1}, x_{1}}^{\#_{1}}}_{\unrhd 0} \underbrace{\mathrm{e}^{-\left(s_{2}-s_{1}\right) \hat{H}_{\mathrm{ext}}}}_{\unrhd 0} \underbrace{\hat{\alpha}_{\varepsilon_{2}, x_{2}}^{\#_{2}}}_{\unrhd 0} \cdots \underbrace{\hat{\alpha}_{\varepsilon_{n}, x_{n}}^{\#_{n}}}_{\unrhd 0} \underbrace{\mathrm{e}^{-\left(\beta-s_{n}\right) \hat{H}_{\mathrm{ext}}}}_{\unrhd 0} \unrhd 0 \quad \text { w.r.t. } \mathfrak{B}_{\mathrm{ext},+}
\end{aligned}
$$

by Propositions 6.17 and 6.18, Thus, by Proposition A.1, we obtain Theorem 6.3, 


\subsection{Proof of Theorem 6.5 and Corollary 6.6}

If $U_{x}=0$, then we have $H=-T$, where $T$ is given by (6.27). Thus, instead of Proposition 6.17, we have the following:

Proposition 6.19 We have $\mathrm{e}^{-\beta H_{\mathrm{ext}}} \unrhd 0$ w.r.t. $\mathfrak{B}_{\mathrm{ext},+}$ for all $\beta \geq 0$.

Note that the unitary operator $\mathscr{U}$ is unnecessary to prove Proposition 6.19. Hence, instead of (6.46), we obtain

$$
\begin{aligned}
& \prod_{j=1}^{\stackrel{n}{n}}\left[a_{x_{j}}^{\#_{j}}\left(s_{j}\right) \otimes \mathbb{1}+\varepsilon_{j} \mathbb{1} \otimes a_{x_{j}}^{\#_{j}}\left(s_{j}\right)\right] \mathrm{e}^{-\beta H_{\mathrm{ext}}} \\
= & \mathrm{e}^{-s_{1} H_{\mathrm{ext}}}\left[a_{x_{1}}^{\#_{1}} \otimes \mathbb{1}+\varepsilon_{1} \mathbb{1} \otimes a_{x_{1}}^{\#_{1}}\right] \mathrm{e}^{-\left(s_{2}-s_{1}\right) H_{\mathrm{ext}}} \cdots\left[a_{x_{n}}^{\#_{n}} \otimes \mathbb{1}+\varepsilon_{n} \mathbb{1} \otimes a_{x_{n}}^{\#_{n}}\right] \mathrm{e}^{-\left(\beta-s_{n}\right) H_{\mathrm{ext}}} \\
\unrhd & 0 \quad \text { w.r.t. } \mathfrak{B}_{\mathrm{ext},+}
\end{aligned}
$$

This completes the proof of Theorem 6.5. By applying Theorem 2.18, we prove Corollary 6.6 .

\section{$7 \quad$ Hubbard model}

\subsection{Results}

\subsubsection{The finite temperature case}

Let $G=(\Lambda, E)$ be a graph with vertex set $\Lambda$ and edge collection $E$. An edge with end-points $x$ and $y$ will be denoted by $\{x, y\}$. We assume that $\{x, x\} \notin E$ for all $x \in \Lambda$, i.e., any loops are excluded. In this section, we assume the following:

(G. 1) $|\Lambda|$ is even.

(G. 2) $G$ is bipartite, i.e., $\Lambda$ admits a partition into two classes such that every edge has its ends in different classes.

The Hubbard model on $G$ is given by

$$
H=\sum_{\{x, y\} \in E} \sum_{\sigma \in\{\uparrow, \downarrow\}}\left(-t_{x y}\right) c_{x \sigma}^{*} c_{y \sigma}+U \sum_{x \in \Lambda}\left(n_{x \uparrow}-\frac{1}{2}\right)\left(n_{x \downarrow}-\frac{1}{2}\right) .
$$

$H$ acts in the Hilbert space $\mathfrak{H}=\mathfrak{F} \otimes \mathfrak{F}$. $\mathfrak{F}$ is the fermionic Fock space defined by $\mathfrak{F}=\oplus_{n \geq 0} \wedge^{n} \ell^{2}(\Lambda)$, where $\wedge^{n} \ell^{2}(\Lambda)$ is the $n$-fold antisymmetric tensor product of $\ell^{2}(\Lambda)$ with $\wedge^{0} \ell^{2}(\Lambda)=\mathbb{C} . c_{x \sigma}$ is the electron annihilation operator that satisfies the canonical anticommutation relations (CARs):

$$
\left\{c_{x \sigma}, c_{x^{\prime} \sigma^{\prime}}^{*}\right\}=\delta_{x x^{\prime}} \delta_{\sigma \sigma^{\prime}}, \quad\left\{c_{x \sigma}, c_{x^{\prime} \sigma^{\prime}}\right\}=0 .
$$

$n_{x \sigma}=c_{x \sigma}^{*} c_{x \sigma}$ is the number operator at vertex $x \in \Lambda . t_{x y} \in \mathbb{R}$ is the quantum mechanical amplitude of an electron hopping from $y$ to $x$. We assume that

(T) $t_{x y}=t_{y x} \neq 0$ for all $\{x, y\} \in E$. 
$U$ is the strength of the Coulomb repulsion 16 such that

(U) $U \geq 0$.

Since $G$ is bipartite, $\Lambda$ can be divided into two disjoint sets $\Lambda_{e}$ and $\Lambda_{o}$. We set $\mu(x)=0$ if $x \in \Lambda_{e}, \mu(x)=1$ if $x \in \Lambda_{o}$. For each $x \in \Lambda$, define

$$
b_{x}=(-1)^{\mu(x)} c_{x \uparrow}^{*} \gamma_{\uparrow} c_{x \downarrow},
$$

where $\gamma_{\uparrow}=(-\mathbb{1})^{N_{\uparrow}}$ with $N_{\sigma}=\sum_{x \in \Lambda} n_{x \sigma}$. Let

$$
\mathfrak{A}=\operatorname{Coni}\left\{b_{x_{1}}^{\#_{1}} b_{x_{2}}^{\#_{2}} \cdots b_{x_{n}}^{\#_{n}} \mid x_{1}, \ldots, x_{n} \in \Lambda, \#_{1}, \ldots, \#_{n} \in\{+,-\}, n \in \mathbb{N}\right\} .
$$

We use the thermal average associated with the grand canonical Gibbs state at inverse temperature $\beta$ :

$$
\langle X\rangle_{\beta}=\operatorname{Tr}\left[X \mathrm{e}^{-\beta H}\right] / \Xi_{\beta}, \quad \Xi_{\beta}=\operatorname{Tr}\left[\mathrm{e}^{-\beta H}\right] .
$$

For each $\beta>0$, we can verify that $\left\langle n_{x}\right\rangle_{\beta}=1$, where $n_{x}=n_{x \uparrow}+n_{x \downarrow}$. This means that the system at half-filling will be considered.

Theorem 7.1 (First Griffiths inequality) Let $A_{1}, \ldots, A_{n} \in \mathfrak{A}$. For all $0 \leq s_{1} \leq$ $s_{2} \leq \cdots \leq s_{n} \leq \beta$, we have

$$
\left\langle\prod_{j=1}^{n} A_{j}\left(s_{j}\right)\right\rangle_{\beta} \geq 0
$$

where $A(s)=\mathrm{e}^{-s H} A \mathrm{e}^{s H}$.

Example 9 For each $x_{1}, \ldots, x_{n} \in \Lambda, \#_{1}, \ldots, \#_{n} \in\{+,-\}$ and $0 \leq s_{1} \leq s_{2} \leq \cdots \leq$ $s_{n} \leq \beta$, we have

$$
\left\langle b_{x_{1}}^{\#_{1}}\left(s_{1}\right) b_{x_{2}}^{\#_{2}}\left(s_{n}\right) \cdots b_{x_{n}}^{\#_{n}}\left(s_{n}\right)\right\rangle_{\beta} \geq 0
$$

where $b_{x}^{\#}(s)=\mathrm{e}^{-s H} b_{x}^{\#} \mathrm{e}^{s H}$.

To state the second quantum Griffiths inequality, we introduce the following notation:

$$
\langle\langle Y\rangle\rangle_{\beta}=\operatorname{Tr}_{\mathfrak{H} \otimes \mathfrak{H}}\left[Y \mathrm{e}^{-\beta H_{\mathrm{ext}}}\right] / \Xi_{\beta}^{2}, \quad H_{\mathrm{ext}}=H \otimes \mathbb{1}+\mathbb{1} \otimes H .
$$

Theorem 7.2 (Second Griffiths inequality) For each $x \in \Lambda, \varepsilon \in\{ \pm 1\}, \# \in\{ \pm\}, \sigma \in$ $\{\uparrow, \downarrow\}$ and $s \geq 0$, we introduce

$$
\alpha_{x \sigma ; \varepsilon}^{\#}(s)=c_{x \sigma}^{\#}(s) \otimes \mathbb{1}+\varepsilon \gamma \otimes c_{x \sigma}^{\#}(s),
$$

\footnotetext{
${ }^{16}$ All results in this section can be extended to a more general Coulomb interaction of the form $\sum_{x, y \in \Lambda} U_{x y}\left(n_{x \uparrow}-\frac{1}{2}\right)\left(n_{y \downarrow}-\frac{1}{2}\right)$, where $U_{x y}$ is real and positive semidefinite.
} 
where $\gamma=(-\mathbb{1})^{N_{\mathrm{e}}}$ with $N_{\mathrm{e}}=N_{\uparrow}+N_{\downarrow}$ and $c_{x \sigma}^{\#}(s)=\mathrm{e}^{-s H} c_{x \sigma}^{\#} \mathrm{e}^{s H}$. Let $x_{1}, \ldots, x_{n} \in \Lambda$. For each $0 \leq s_{1} \leq s_{2} \leq \cdots \leq s_{n} \leq \beta, \#_{1}, \ldots, \#_{n} \in\{+,-\}$ and $\varepsilon_{1}, \ldots, \varepsilon_{n} \in\{ \pm 1\}$, we have

$$
\left\langle\left\langle\prod_{j=1}^{n}\left[(-1)^{\mu\left(x_{j}\right)} \alpha_{x_{j} \uparrow ; \varepsilon_{j}}^{\#_{j}}\left(s_{j}\right) \gamma_{\uparrow} \otimes \gamma_{\uparrow} \alpha_{x_{j \downarrow} \downarrow \varepsilon_{j}}^{\#_{j}}\left(s_{j}\right)\right]\right\rangle_{\beta} \geq 0\right.
$$

and

$$
\left\langle\left\langle\prod_{j=1}^{n}\left[(-1)^{\mu\left(x_{j}\right)} \alpha_{x_{j} \uparrow ; \varepsilon_{j}}^{\#_{j}}\left(s_{j}\right) \gamma_{\uparrow} \otimes \gamma_{\uparrow} \alpha_{x_{j \downarrow} ;-\varepsilon_{j}}^{\#_{j}}\left(s_{j}\right)\right]\right\rangle_{\beta} \geq 0 .\right.
$$

Corollary 7.3 Let $x_{1}, \ldots, x_{n} \in \Lambda$. For each $0 \leq s_{1} \leq s_{2} \leq \cdots \leq s_{n} \leq \beta$ and $\#_{1}, \ldots, \#_{n} \in\{+,-\}$, we have

$$
\left\langle\left\langle\prod_{j=1}^{n}\left[b_{x_{j}}^{\#_{j}}\left(s_{j}\right) \otimes \gamma_{\uparrow}-\gamma_{\uparrow} \otimes b_{x_{j}}^{\#_{j}}\left(s_{j}\right)\right]\right\rangle_{\beta} \geq 0 .\right.
$$

Corollary 7.4 Let $x_{1}, \ldots, x_{2 n} \in \Lambda$. For each $0 \leq s_{1} \leq s_{2} \leq \cdots \leq s_{2 n} \leq \beta$ and $\#_{1}, \ldots, \#_{2 n} \in\{+,-\}$, we have

$$
\begin{aligned}
& \left\langle\left\langle\prod_{j=1}^{2 n}\left[b_{x_{j}}^{\#_{j}}\left(s_{j}\right) \otimes \gamma_{\uparrow}-\gamma_{\uparrow} \otimes b_{x_{j}}^{\#_{j}}\left(s_{j}\right)\right]\right\rangle\right\rangle_{\beta} \\
\geq & \left\langle\left\langle\prod_{j=1}^{2 n}(-1)^{\mu\left(x_{j}\right)}\left[c_{x_{j} \uparrow}^{\#_{j}}\left(s_{j}\right) \gamma_{\downarrow} \otimes \gamma_{\uparrow} c_{x_{j} \downarrow}^{\#_{j}}\left(s_{j}\right)-\gamma_{\downarrow} c_{x_{j \downarrow}}^{\#_{j}}\left(s_{j}\right) \otimes c_{x_{j} \uparrow}^{\#_{j}}\left(s_{j}\right) \gamma_{\uparrow}\right]\right\rangle\right\rangle_{\beta} \\
\geq & 0 .
\end{aligned}
$$

Example 10 Consider the case where $n=2$. We then have

$$
\begin{aligned}
& (-1)^{\mu(x)+\mu(y)}\left(\left\langle c_{x \downarrow}^{*} c_{x \uparrow} c_{y \uparrow}^{*} c_{y \downarrow}\right\rangle_{\beta}-\left\langle c_{x \downarrow}^{*} c_{x \uparrow}\right\rangle_{\beta}\left\langle c_{y \uparrow}^{*} c_{y \downarrow}\right\rangle_{\beta}\right) \\
\geq & (-1)^{\mu(x)+\mu(y)}\left(\left\langle c_{x \uparrow} c_{y \uparrow}^{*}\right\rangle_{\beta}\left\langle c_{x \downarrow}^{*} c_{y \downarrow}\right\rangle_{\beta}+\left\langle c_{x \uparrow} c_{y \downarrow}\right\rangle_{\beta}\left\langle c_{x \downarrow}^{*} c_{y \uparrow}^{*}\right\rangle_{\beta}\right) \\
\geq & 0 .
\end{aligned}
$$

Since $\left\langle c_{x \downarrow}^{*} c_{x \uparrow}\right\rangle_{\beta}=0=\left\langle c_{x \uparrow} c_{y \downarrow}\right\rangle_{\beta}$ by the symmetries of the system, we arrive at

$$
(-1)^{\mu(x)+\mu(y)}\left\langle c_{x \downarrow}^{*} c_{x \uparrow} c_{y \uparrow}^{*} c_{y \downarrow}\right\rangle_{\beta} \geq(-1)^{\mu(x)+\mu(y)}\left\langle c_{x \uparrow} c_{y \uparrow}^{*}\right\rangle_{\beta}\left\langle c_{x \downarrow}^{*} c_{y \downarrow}\right\rangle_{\beta} \geq 0 .
$$

If $x, y \in \Lambda_{e}$ or $x, y \in \Lambda_{o}$, then $(-1)^{\mu(x)+\mu(y)}=1$, so that we obtain a standard-type correlation inequality. $\diamond$ 
Corollary 7.5 Let $\bar{n}_{x \uparrow}=\mathbb{1}-n_{x \uparrow}$. Let $x_{1}, \ldots, x_{n} \in \Lambda$. We have

$$
\begin{aligned}
& \left\langle\left\langle\prod_{j=1}^{n}\left[\bar{n}_{x_{j} \uparrow} n_{x_{j \downarrow}} \otimes \mathbb{1}+\mathbb{1} \otimes \bar{n}_{x_{j} \uparrow} n_{x_{j} \downarrow}\right]\right\rangle\right\rangle_{\beta} \\
\geq & \left\langle\left\langle\prod_{j=1}^{n}\left[\bar{n}_{x_{j} \uparrow} \otimes n_{x_{j \downarrow} \downarrow}+n_{x_{j \downarrow}} \otimes \bar{n}_{x_{j} \uparrow}\right]\right\rangle\right\rangle_{\beta} \\
\geq & 0 .
\end{aligned}
$$

Example 11 In the case where $n=2$, we have

$$
\begin{aligned}
& \left\langle\bar{n}_{x \uparrow} \bar{n}_{y \uparrow} n_{x \downarrow} n_{y \downarrow}\right\rangle_{\beta}+\left\langle\bar{n}_{x \uparrow} n_{x \downarrow}\right\rangle_{\beta}\left\langle\bar{n}_{y \uparrow} n_{y \downarrow}\right\rangle_{\beta} \\
& -\left\langle\bar{n}_{x \uparrow} \bar{n}_{y \uparrow}\right\rangle_{\beta}\left\langle n_{x \downarrow} n_{y \downarrow}\right\rangle_{\beta}-\left\langle\bar{n}_{x \uparrow} n_{y \downarrow}\right\rangle_{\beta}\left\langle\bar{n}_{y \uparrow} n_{x \downarrow}\right\rangle_{\beta} \geq 0 . \diamond
\end{aligned}
$$

Remark 7.6 Our results can be extended to a general class of electron-phonon(or photon) Hamiltonians, including the Holstein-Hubbard model and the SSH model. $\diamond$

\subsubsection{The zero-temperature case}

Our results can be extended to the case where $\beta=\infty$. Unfortunately, the general theorems in Section 3 cannot be directly applied to this model. To clarify the main points of modification, we state results without proofs.

We assume an additional condition.

(G. 3) $G$ is connected, i.e., any of its vertices are linked by a path in $G$.

We consider a half-filled system. Thus, our Hilbert space is restricted to

$$
\mathfrak{E}=\mathfrak{H} \cap \operatorname{ker}\left(N_{\mathrm{e}}-|\Lambda|\right)
$$

Let $S^{(z)}=\frac{1}{2}\left(N_{\uparrow}-N_{\downarrow}\right)$. Since $S^{(z)}$ commutes with $H$, we have the following decomposition:

$$
\mathfrak{E}=\bigoplus_{M=-|\Lambda| / 2}^{|\Lambda| / 2} \mathfrak{E}_{M}, \quad \mathfrak{E}_{M}=\mathfrak{E} \cap \operatorname{ker}\left(S^{(z)}-M\right)
$$

$\mathfrak{E}_{M}$ is called the $M$-subspace. For each $M \in \operatorname{spec}\left(S^{(z)}\right)$, set $H_{M}=H \uparrow \mathfrak{E}_{M}$. The following theorem is important.

Theorem 7.7 [38, 44] For each $M \in\{-|\Lambda| / 2,-(|\Lambda|-2) / 2, \ldots,|\Lambda| / 2\}, H_{M}$ has a unique ground state.

We denote the normalized ground state of $H_{M}$ by $\psi_{M}$. We define the ground state expectation value by

$$
\langle X\rangle_{\infty, M}=\left\langle\psi_{M} \mid X \psi_{M}\right\rangle
$$


Theorem 7.8 Let $A_{1}, \ldots, A_{n} \in \mathfrak{A}$. For all $0 \leq s_{1} \leq s_{2} \leq \cdots \leq s_{n}$, we have

$$
\left\langle\prod_{j=1}^{\vec{n}} A_{j}\left(s_{j}\right)\right\rangle_{\infty, M} \geq 0
$$

We introduce the following notation:

$$
\langle\langle Y\rangle\rangle_{\infty, M}=\left\langle\psi_{M} \otimes \psi_{M} \mid Y \psi_{M} \otimes \psi_{M}\right\rangle .
$$

Theorem 7.9 Let $x_{1}, \ldots, x_{n} \in \Lambda$. For each $0 \leq s_{1} \leq s_{2} \leq \cdots \leq s_{n}, \#_{1}, \ldots, \#_{n} \in$ $\{+,-\}$ and $\varepsilon_{1}, \ldots, \varepsilon_{n} \in\{ \pm 1\}$, we have

$$
\left\langle\left\langle\prod_{j=1}^{n}\left[(-1)^{\mu\left(x_{j}\right)} \alpha_{x_{j} \uparrow ; \varepsilon_{j}}^{\#_{j}}\left(s_{j}\right) \gamma_{\uparrow} \otimes \gamma_{\uparrow} \alpha_{x_{j \downarrow} \downarrow \varepsilon_{j}}^{\#_{j}}\left(s_{j}\right)\right]\right\rangle\right\rangle_{\infty, M} \geq 0
$$

and

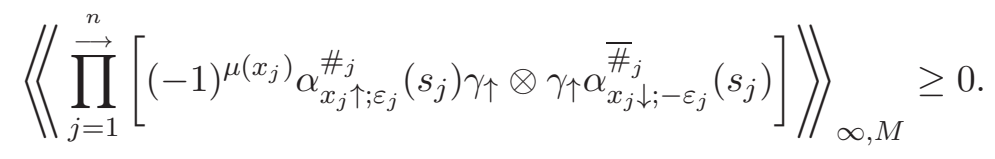

\subsection{Proof of Theorem 7.1}

The hole-particle transformation $\mathcal{U}$ is a unitary operator such that

$$
\mathcal{U} c_{x \uparrow} \mathcal{U}^{*}=(-1)^{\mu(x)} c_{x \uparrow}^{*}, \quad \mathcal{U} c_{x \downarrow} \mathcal{U}^{*}=c_{x \downarrow} .
$$

Let $\hat{H}=\mathcal{U} H \mathcal{U}^{*}$. Then we obtain the attractive Hubbard model:

$$
\hat{H}=\sum_{\{x, y\} \in E} \sum_{\sigma \in\{\uparrow, \downarrow\}}\left(-t_{x y}\right) c_{x \sigma}^{*} c_{y \sigma}-U \sum_{x \in \Lambda}\left(n_{x \uparrow}-\frac{1}{2}\right)\left(n_{x \downarrow}-\frac{1}{2}\right) .
$$

Let $c_{x}$ be the annihilation operator on $\mathfrak{F}$. We note that

$$
c_{x \uparrow}=c_{x} \otimes \mathbb{1}, \quad c_{x \downarrow}=(-\mathbb{1})^{\mathrm{N}} \otimes c_{x},
$$

where $\mathrm{N}=\sum_{x \in \Lambda} c_{x}^{*} c_{x}$. Then we obtain

$$
\hat{H}=\mathbf{T} \otimes \mathbb{1}+\mathbb{1} \otimes \mathrm{T}-U \sum_{x \in \Lambda}\left(\mathrm{n}_{x}-\frac{1}{2}\right) \otimes\left(\mathrm{n}_{x}-\frac{1}{2}\right),
$$

where $\mathrm{n}_{x}=c_{x}^{*} c_{x}$ and

$$
\mathrm{T}=\sum_{\{x, y\} \in E}\left(-t_{x y}\right) c_{x}^{*} c_{y} .
$$

Let $\vartheta_{1}$ be an antilinear involution on $\mathfrak{F}$ defined by

$$
\vartheta_{1} c_{x_{1}}^{*} \cdots c_{x_{n}}^{*} \Omega=c_{x_{1}}^{*} \cdots c_{x_{n}}^{*} \Omega, \quad x_{1}, \ldots, x_{n} \in \Lambda,
$$

where $\Omega$ is the Fock vacuum in $\mathfrak{F}$. By (3.4), we have the following identification:

$$
\mathfrak{H}=\mathscr{L}^{2}(\mathfrak{F}) .
$$

Moreover, by (3.4) and (77.28), we obtain the following: 
Proposition 7.10 We have

$$
\hat{H}=\mathcal{L}(\mathrm{T})+\mathcal{R}(\mathrm{T})-U \sum_{x \in \Lambda} \mathcal{L}\left(\mathrm{n}_{x}-\frac{1}{2}\right) \mathcal{R}\left(\mathrm{n}_{x}-\frac{1}{2}\right),
$$

Proposition 7.11 We have the following:

(i) $\hat{b}_{x}:=\mathcal{U} b_{x} \mathcal{U}^{*} \succeq 0$ w.r.t. $\mathscr{L}^{2}(\mathfrak{F})_{+}$for all $x \in \Lambda$.

(ii) $\mathrm{e}^{-\beta \hat{H}} \succeq 0$ w.r.t. $\mathscr{L}^{2}(\mathfrak{F})_{+}$for all $\beta \geq 0$.

Proof. (i) This immediately follows from the identification $\hat{b}_{x}=\mathcal{L}\left(c_{x}\right) \mathcal{R}\left(c_{x}^{*}\right)$.

(ii) By Proposition 7.10 and Corollary A.9, we obtain (ii)

Corollary 7.12 For all $A \in \mathfrak{A}$, we have $\mathcal{U A U}^{*} \succeq 0$ w.r.t. $\mathscr{L}^{2}(\mathfrak{F})_{+}$.

\subsubsection{Completion of proof of Theorem 7.1}

By Theorem 3.11 and Corollary 7.12 , we obtain Theorem 7.1 ,

\subsection{Proof of Theorem 7.2}

Let $\mathfrak{H}_{\text {ext }}=\mathfrak{H} \otimes \mathfrak{H}$. Let

$$
\phi_{x \sigma}=\frac{1}{\sqrt{2}}\left(c_{x \sigma} \otimes \mathbb{1}+\gamma \otimes c_{x \sigma}\right), \quad \psi_{x \sigma}=\frac{1}{\sqrt{2}}\left(c_{x \sigma} \otimes \mathbb{1}-\gamma \otimes c_{x \sigma}\right) .
$$

$\phi_{x \sigma}$ and $\psi_{x \sigma}$ act in $\mathfrak{H}_{\text {ext }}$ as well. These operators satisfy the following CARs:

$$
\begin{array}{ll}
\left\{\phi_{x \sigma}, \phi_{y \sigma^{\prime}}^{*}\right\}=\delta_{x y} \delta_{\sigma \sigma^{\prime}}, & \left\{\phi_{x \sigma}, \phi_{y \sigma^{\prime}}\right\}=0, \\
\left\{\psi_{x \sigma}, \psi_{y \sigma^{\prime}}^{*}\right\}=\delta_{x y} \delta_{\sigma \sigma^{\prime}}, & \left\{\psi_{x \sigma}, \psi_{y \sigma^{\prime}}\right\}=0, \\
\left\{\phi_{x \sigma}, \psi_{y \sigma^{\prime}}^{*}\right\}=0, & \left\{\phi_{x \sigma}, \psi_{y \sigma^{\prime}}\right\}=0 .
\end{array}
$$

Let $\left\{\phi_{x}, \psi_{x} \mid x \in \Lambda\right\}$ be new annihilation operators on $\mathfrak{X}=\mathfrak{F} \otimes \mathfrak{F}$ such that

$$
\begin{aligned}
& \left\{\phi_{x}, \phi_{y}^{*}\right\}=\delta_{x y}, \quad\left\{\phi_{x}, \phi_{y}\right\}=0, \\
& \left\{\psi_{x}, \psi_{y}^{*}\right\}=\delta_{x y}, \quad\left\{\psi_{x}, \psi_{y}\right\}=0, \\
& \left\{\phi_{x}, \psi_{y}^{*}\right\}=0, \quad\left\{\phi_{x}, \psi_{y}\right\}=0,
\end{aligned}
$$

and $\phi_{x} \Omega_{\mathfrak{X}}=0=\psi_{\mathfrak{x}} \Omega_{\mathfrak{X}}$, where $\Omega_{\mathfrak{X}}$ is the Fock vacuum in $\mathfrak{X}$. Then we have the following identifications:

$$
\phi_{x \uparrow}=\phi_{x} \otimes \mathbb{1}, \quad \phi_{x \downarrow}=(-\mathbb{1})^{\mathcal{N}} \otimes \phi_{x}, \quad \psi_{x \uparrow}=\psi_{x} \otimes \mathbb{1}, \quad \psi_{x \downarrow}=(-\mathbb{1})^{\mathcal{N}} \otimes \psi_{x},
$$

where $\mathcal{N}=\sum_{x \in \Lambda}\left(\phi_{x}^{*} \phi_{x}+\psi_{x}^{*} \psi_{x}\right)$. Let

$$
\mathscr{U}=\mathcal{U} \otimes \mathcal{U}
$$

Set

$$
\hat{H}_{\mathrm{ext}}=\mathscr{U} H_{\mathrm{ext}} \mathscr{U}^{*}+\frac{1}{2} U|\Lambda| .
$$


Then $\hat{H}_{\text {ext }}$ can be expressed as

$$
\hat{H}_{\mathrm{ext}}=\mathbb{T} \otimes \mathbb{1}+\mathbb{1} \otimes \mathbb{T}-\mathbb{V}
$$

where

$$
\begin{aligned}
\mathbb{T} & =\sum_{\{x, y\} \in E}\left(-t_{x y}\right)\left(\phi_{x}^{*} \phi_{y}+\psi_{x}^{*} \psi_{y}\right)+\frac{U}{2} \mathcal{N}, \\
\mathbb{V} & =\frac{U}{2} \sum_{x \in \Lambda}\left(\mathcal{N}_{x} \otimes \mathcal{N}_{x}+\mathcal{M}_{x} \otimes \mathcal{M}_{x}\right), \\
\mathcal{N}_{x} & =\phi_{x}^{*} \phi_{x}+\psi_{x}^{*} \psi_{x}, \quad \mathcal{M}_{x}=\phi_{x}^{*} \psi_{x}+\psi_{x}^{*} \phi_{x} .
\end{aligned}
$$

Let $\vartheta_{2}$ be an antilinear involution on $\mathfrak{X}$ defined by

$$
\vartheta_{2} \phi_{x} \vartheta_{2}=\phi_{x}, \quad \vartheta_{2} \psi_{x} \vartheta_{2}=\psi_{x}, \quad \vartheta_{2} \Omega_{\mathfrak{X}}=\Omega_{\mathfrak{X}} .
$$

By (3.4), we have the identification

$$
\mathfrak{H}_{\text {ext }}=\mathscr{L}^{2}(\mathfrak{X}) .
$$

In addition, we have the following expression:

Proposition 7.13 We have $\hat{H}_{\text {ext }}=\mathcal{L}(\mathbb{T})+\mathcal{R}(\mathbb{T})-\mathbb{V}$, where

$$
\mathbb{V}=\frac{U}{2} \sum_{x \in \Lambda}\left\{\mathcal{L}\left(\mathcal{N}_{x}\right) \mathcal{R}\left(\mathcal{N}_{x}\right)+\mathcal{L}\left(\mathcal{M}_{x}\right) \mathcal{R}\left(\mathcal{M}_{x}\right)\right\} .
$$

By Corollary A.9, we obtain the following:

Corollary 7.14 For all $\beta \geq 0$, we have $\exp \left(-\beta \hat{H}_{\text {ext }}\right) \succeq 0$ w.r.t. $\mathscr{L}^{2}(\mathfrak{X})_{+}$.

The below proposition immediately follows from the definitions (note that $\mathscr{U} \gamma \otimes$ $\mathbb{1} \mathscr{U}^{*}=\gamma \otimes \mathbb{1}$ by (G. 1)).

Proposition 7.15 We have the following:

(i) $\mathscr{U}(-1)^{\mu(x)}\left(c_{x \uparrow}^{\#} \otimes \mathbb{1}+\gamma \otimes c_{x \uparrow}^{\#}\right) \mathscr{U}^{*}=\sqrt{2} \mathcal{L}\left(\phi_{x}^{\#}\right)$.

(ii) $\mathscr{U}(-1)^{\mu(x)}\left(c_{x \uparrow}^{\#} \otimes \mathbb{1}-\gamma \otimes c_{x \uparrow}^{\#}\right) \mathscr{U}^{*}=\sqrt{2} \mathcal{L}\left(\psi_{x}^{\#}\right)$.

(iii) $\mathscr{U} \gamma_{\uparrow} \otimes \gamma_{\uparrow}\left(c_{x \downarrow}^{\#} \otimes \mathbb{1}+\gamma \otimes c_{x \downarrow}^{\#}\right) \mathscr{U}^{*}=\sqrt{2} \mathcal{R}\left(\phi_{x}^{\#}\right)$.

(iv) $\mathscr{U} \gamma_{\uparrow} \otimes \gamma_{\uparrow}\left(c_{x \downarrow}^{\#} \otimes \mathbb{1}-\gamma \otimes c_{x \downarrow}^{\#}\right) \mathscr{U}^{*}=\sqrt{2} \mathcal{R}\left(\psi_{x}^{\#}\right)$.

Corollary 7.16 Let

$$
\alpha_{x \sigma ; \varepsilon}=c_{x \sigma} \otimes \mathbb{1}+\varepsilon \gamma \otimes c_{x \sigma} .
$$

For all $\varepsilon \in\{ \pm 1\}, \# \in\{ \pm\}$ and $x \in \Lambda$, we have

$$
\mathscr{U}(-1)^{\mu(x)} \alpha_{x \uparrow, \varepsilon}^{\#} \gamma_{\uparrow} \otimes \gamma_{\uparrow} \alpha_{x \downarrow, \varepsilon}^{\#} \mathscr{U}^{*} \succeq 0
$$

w.r.t. $\mathscr{L}^{2}(\mathfrak{X})_{+}$. 
Proof. By Proposition 7.15, we have

$$
\begin{aligned}
& \mathscr{U}(-1)^{\mu(x)} \alpha_{x \uparrow,-1}^{\#} \gamma_{\uparrow} \otimes \gamma_{\uparrow} \alpha_{x \downarrow,-1}^{\#} \mathscr{U}^{*}=2 \mathcal{L}\left(\psi_{x}^{\#}\right) \mathcal{R}\left(\psi_{x}^{\#}\right) \succeq 0 \quad \text { w.r.t. } \mathscr{L}^{2}(\mathfrak{X})_{+}, \\
& \mathscr{U}(-1)^{\mu(x)} \alpha_{x \uparrow,+1}^{\#} \gamma_{\uparrow} \otimes \gamma_{\uparrow} \alpha_{x \downarrow,+1}^{\#} \mathscr{U}^{*}=2 \mathcal{L}\left(\phi_{x}^{\#}\right) \mathcal{R}\left(\phi_{x}^{\#}\right) \succeq 0 \quad \text { w.r.t. } \mathscr{L}^{2}(\mathfrak{X})_{+}
\end{aligned}
$$

\subsubsection{Completion of proof of Theorem 7.2}

Proof of 7.10

Let $D_{\varepsilon, \#, x}=(-1)^{\mu(x)} \alpha_{x \uparrow, \varepsilon}^{\#} \gamma_{\uparrow} \otimes \gamma_{\uparrow} \alpha_{x \downarrow, \varepsilon}^{\#}$. Then we see that by Corollaries 7.14 and 7.16,

$$
\begin{aligned}
& \mathscr{U}\left[\prod_{j=1}^{\vec{n}}(-1)^{\mu\left(x_{j}\right)} \alpha_{x_{j} \uparrow ; \varepsilon_{j}}^{\#_{j}}\left(s_{j}\right) \gamma_{\uparrow} \otimes \gamma_{\uparrow} \alpha_{x_{j} \downarrow ; \varepsilon_{j}}^{\#_{j}}\left(s_{j}\right)\right] \mathrm{e}^{-\beta H_{\mathrm{ext}} \mathscr{U}^{*}} \\
= & \underbrace{\mathrm{e}^{-s_{1} \hat{H}_{\mathrm{ext}}}}_{\succeq 0} \underbrace{\mathscr{U} D_{\varepsilon_{1}, \#_{1}, x_{1}} \mathscr{U}^{*}}_{\succeq 0} \underbrace{\mathrm{e}^{-\left(s_{2}-s_{1}\right) \hat{H}_{\mathrm{ext}}}}_{\succeq 0} \cdots \underbrace{\mathscr{U} D_{\varepsilon_{n}, \#_{n}, x_{n}} \mathscr{U}^{*}}_{\succeq 0} \underbrace{\mathrm{e}^{-\left(\beta-s_{n}\right) \hat{H}_{\mathrm{ext}}}}_{\succeq 0} \succeq 0
\end{aligned}
$$

w.r.t. $\mathscr{L}^{2}(\mathfrak{X})_{+}$. Thus, by Theorem 3.10 , we obtain (7.10).

Proof of (7.11)

Let $\mathscr{Q}$ be a unitary operator defined by $\mathscr{Q}=\mathbb{1} \otimes(-\mathbb{1})^{N_{\downarrow}}$. Then we see that

$$
\mathscr{Q} H_{\text {ext }} \mathscr{Q}^{-1}=H_{\text {ext }}, \quad \mathscr{Q} \alpha_{x \uparrow, \varepsilon} \mathscr{Q}^{-1}=\alpha_{x \uparrow, \varepsilon}, \quad \mathscr{Q} \alpha_{x \downarrow, \varepsilon} \mathscr{Q}^{-1}=\alpha_{x \downarrow,-\varepsilon} .
$$

Thus, (7.11) follows from (7.10).

\subsection{Proof of Corollary 7.3}

Lemma 7.17 Let $C_{x, \varepsilon}=(-1)^{\mu(x)} \alpha_{x \uparrow, \varepsilon}^{\#} \gamma_{\uparrow} \otimes \gamma_{\uparrow} \alpha_{x \downarrow,-\varepsilon}^{\#}$. Set $\mathscr{W}=\mathscr{U} \mathscr{Q}$. Then we obtain $\mathscr{W} C_{x, \varepsilon} \mathscr{W}^{-1} \succeq 0$ w.r.t. $\mathscr{L}^{2}(\mathfrak{X})_{+}$.

Proof. By Corollary 7.16 and (7.55), we see that

$$
\mathscr{W} C_{x, \varepsilon} \mathscr{W}^{-1}=\mathscr{U} D_{\varepsilon,+1, x} \mathscr{U}^{-1} \succeq 0 \quad \text { w.r.t. } \mathscr{L}^{2}(\mathfrak{X})_{+} .
$$

Lemma 7.18 For all $\beta \geq 0$, we have $\mathscr{W} \mathrm{e}^{-\beta H_{\text {ext }} \mathscr{W}^{-1}} \succeq 0$ w.r.t. $\mathscr{L}^{2}(\mathfrak{X})_{+}$.

Proof. Since $\mathscr{Q} H_{\text {ext }} \mathscr{Q}^{-1}=H_{\text {ext }}$, we see that $\mathscr{W} \mathrm{e}^{-\beta H_{\text {ext }} \mathscr{W}^{-1}}=\mathrm{e}^{-\beta \hat{H}_{\text {ext }}} \succeq 0$ w.r.t. $\mathscr{L}^{2}(\mathfrak{X})_{+}$. 


\subsubsection{Completion of proof of Corollary 7.3}

By Lemma 7.17, we obtain

$$
\mathscr{W} C_{x, \varepsilon} \mathscr{W}^{-1}=\mathscr{W} A_{x} \mathscr{W}^{-1}+\varepsilon \mathscr{W} B_{x} \mathscr{W}^{-1} \succeq 0 \quad \text { w.r.t. } \mathscr{L}^{2}(\mathfrak{X})_{+},
$$

where

$$
\begin{aligned}
& A_{x}=\mathscr{Q}^{-1}\left(b_{x} \otimes \gamma_{\uparrow}-\gamma_{\uparrow} \otimes b_{x}\right) \mathscr{Q}, \\
& B_{x}=-(-1)^{\mu(x)} \mathscr{Q}^{-1}\left(c_{x \uparrow}^{*} \gamma_{\downarrow} \otimes \gamma_{\uparrow} c_{x \downarrow}-\gamma_{\downarrow} c_{x \downarrow} \otimes c_{x \uparrow}^{*} \gamma_{\uparrow}\right) \mathscr{Q} .
\end{aligned}
$$

Thus, we have

$$
\mathscr{W} A_{x} \mathscr{W}^{-1}=\frac{1}{2} \underbrace{\mathscr{W} C_{x,+} \mathscr{W}^{-1}}_{\succeq 0}+\frac{1}{2} \underbrace{\mathscr{W} C_{x,-} \mathscr{W}^{-1}}_{\succeq 0} \succeq 0 \quad \text { w.r.t. } \mathscr{L}^{2}(\mathfrak{X})_{+} .
$$

Finally, observe that

$$
\begin{aligned}
& \mathscr{W}\left[\prod_{j=1}^{n} A_{x_{j}}^{\# H_{j}}\left(s_{j}\right)\right] \mathrm{e}^{-\beta H_{\mathrm{ext}} \mathscr{W}^{-1}} \\
= & \underbrace{\mathscr{W} \mathrm{e}^{-s_{1} H_{\mathrm{ext}} \mathscr{W}^{-1}}}_{\succeq 0} \underbrace{\mathscr{W} A_{x_{1}}^{\#_{1}} \mathscr{W}^{-1}}_{\succeq 0} \underbrace{\mathscr{W} \mathrm{e}^{-\left(s_{2}-s_{1}\right) H_{\mathrm{ext}} \mathscr{W}^{-1}}}_{\succeq 0} \cdots \underbrace{\mathscr{W} \mathrm{e}^{-\left(\beta-s_{n}\right) H_{\mathrm{ext}}} \mathscr{W}^{-1}}_{\succeq 0} \succeq 0
\end{aligned}
$$

w.r.t. $\mathscr{L}^{2}(\mathfrak{X})_{+}$. By Theorem 3.10 , we conclude Corollary 7.3 ,

\subsection{Proof of Corollary 7.4}

\subsubsection{First part of the proof}

Note that $\mathscr{W} B_{x} \mathscr{W}^{-1}=\frac{1}{2} \mathscr{W} C_{x,+} \mathscr{W}^{-1}-\frac{1}{2} \mathscr{W} C_{x,-} \mathscr{W}^{-1}$. Combining this with (7.60), we have

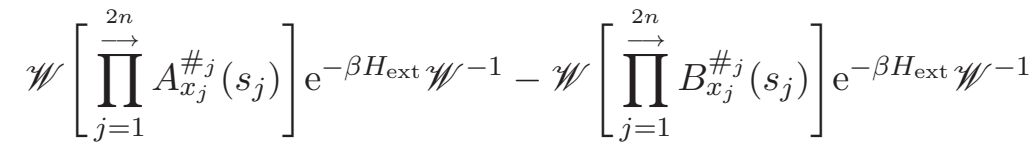

$$
\begin{aligned}
& =\sum_{\delta_{1}, \ldots, \delta_{n} \in\{ \pm\}} X_{\delta_{1}, \ldots, \delta_{n}} \underbrace{\mathscr{W} C_{x_{1}, \delta_{1}}\left(s_{1}\right) \cdots C_{x_{n}, \delta_{n}}\left(s_{n}\right) \mathrm{e}^{\beta H_{\mathrm{ext}} \mathscr{W}^{-1}}}_{\succeq 0},
\end{aligned}
$$

where each $X_{\delta_{1}, \ldots, \delta_{n}}$ is a positive constant. Thus, the RHS of $(\overline{7.62}) \succeq 0$ w.r.t. $\mathscr{L}^{2}(\mathfrak{X})_{+}$. By Theorem 3.10, we obtain the first inequality in (7.13).

\subsubsection{Second part of the proof}

We will show the second inequality in (7.13). Let $\Theta$ be an antilinear involution on $\mathfrak{H}$ such that

$$
\Theta c_{x \sigma} \Theta=c_{x \sigma}, \quad \Theta \Omega_{\mathfrak{H}}=\Omega_{\mathfrak{H}},
$$

where $\Omega_{\mathfrak{H}}=\Omega \otimes \Omega$. Then by (3.4), we have $\mathfrak{H}_{\text {ext }}=\mathscr{L}^{2}(\mathfrak{H})$ and

$$
\hat{H}_{\text {ext }}=\mathcal{L}(\hat{H})+\mathcal{R}(\hat{H}) .
$$

By Corollary A.9, we have the following: 
Proposition 7.19 For all $\beta \geq 0$, we have $\exp \left(-\beta \hat{H}_{\text {ext }}\right) \succeq 0$ w.r.t. $\mathscr{L}^{2}(\mathfrak{H})_{+}$.

Let $S$ be the unitary operator on $\mathfrak{H}$ given by

$$
S c_{x \uparrow} S^{-1}=c_{x \downarrow}, \quad S c_{x \downarrow} S^{-1}=c_{x \uparrow} .
$$

Set $\mathscr{R}=\mathbb{1} \otimes S \mathscr{U}$. Remark that since $S \hat{H} S^{-1}=\hat{H}$, we know that

$$
\mathscr{R} \mathrm{e}^{-\beta H_{\text {ext }}} \mathscr{R}^{-1}=\mathrm{e}^{-\beta \hat{H}_{\text {ext }}} \succeq 0 \text { w.r.t. } \mathscr{L}^{2}(\mathfrak{H})_{+}
$$

by Proposition 7.19.

Proposition 7.20 We have the following:

(i) $\mathscr{R}(-1)^{\mu(x)} c_{x \uparrow} \gamma_{\downarrow} \otimes \mathbb{1} \mathscr{R}^{-1}=\mathcal{L}\left(c_{x \uparrow}^{*} \gamma_{\downarrow}\right)$.

(ii) $\mathscr{R} c_{x \downarrow} \gamma_{\downarrow} \otimes \mathbb{1} \mathscr{R}^{-1}=\mathcal{L}\left(c_{x \downarrow} \gamma_{\downarrow}\right)$.

(iii) $\mathscr{R}(-1)^{\mu(x)} \mathbb{1} \otimes c_{x \uparrow} \gamma_{\uparrow} \mathscr{R}^{-1}=\mathcal{R}\left(\gamma_{\downarrow} c_{x \downarrow}\right)$.

(iv) $\mathscr{R} \mathbb{1} \otimes \gamma_{\uparrow} c_{x \downarrow} \mathscr{R}^{-1}=\mathcal{R}\left(\gamma_{\downarrow} c_{x \uparrow}^{*}\right)$.

Corollary 7.21 We have the following:

(i) $\mathscr{R}(-1)^{\mu(x)} c_{x \uparrow}^{*} \gamma_{\downarrow} \otimes \gamma_{\uparrow} c_{x \downarrow} \mathscr{R}^{-1} \succeq 0$ w.r.t. $\mathscr{L}^{2}(\mathfrak{H})_{+}$.

(ii) $-\mathscr{R}(-1)^{\mu(x)} \gamma_{\downarrow} c_{x \downarrow} \otimes c_{x \uparrow}^{*} \gamma_{\uparrow} \mathscr{R}^{-1} \succeq 0$ w.r.t. $\mathscr{L}^{2}(\mathfrak{H})_{+}$.

Proof. By Proposition 7.20 , we see that

$$
\begin{aligned}
\mathscr{R}(-1)^{\mu(x)} c_{x \uparrow}^{*} \gamma_{\downarrow} \otimes \gamma_{\uparrow} c_{x \downarrow} \mathscr{R}^{-1} & =\mathcal{L}\left(c_{x \uparrow} \gamma_{\downarrow}\right) \mathcal{R}\left(\gamma_{\downarrow} c_{x \uparrow}^{*}\right) \succeq 0 \quad \text { w.r.t. } \mathscr{L}^{2}(\mathfrak{H})_{+}, \\
-\mathscr{R}(-1)^{\mu(x)} \gamma_{\downarrow} c_{x \downarrow} \otimes c_{x \uparrow}^{*} \gamma_{\uparrow} \mathscr{R}^{-1} & =\mathcal{L}\left(c_{x \downarrow} \gamma_{\downarrow}\right) \mathcal{R}\left(\left(c_{x \downarrow} \gamma_{\downarrow}\right)^{*}\right) \succeq 0 \quad \text { w.r.t. } \mathscr{L}^{2}(\mathfrak{H})_{+} .
\end{aligned}
$$

This completes the proof.

Set

$$
K_{x}=(-1)^{\mu(x)}\left(c_{x \uparrow}^{*} \gamma_{\downarrow} \otimes \gamma_{\uparrow} c_{x \downarrow}-\gamma_{\downarrow} c_{x \downarrow} \otimes c_{x \uparrow}^{*} \gamma_{\uparrow}\right) .
$$

By Corollary 17.21, we know that $\mathscr{R} K_{x} \mathscr{R}^{-1} \succeq 0$ w.r.t. $\mathscr{L}^{2}(\mathfrak{H})_{+}$. Thus, by (17.66), we obtain

$$
\begin{aligned}
& \mathscr{R} \prod_{j=1}^{2 n}(-1)^{\mu\left(x_{j}\right)}\left[c_{x_{j} \uparrow}^{\#_{j}}\left(s_{j}\right) \gamma_{\downarrow} \otimes \gamma_{\uparrow} c_{x_{j \downarrow} \downarrow}^{\#_{j}}\left(s_{j}\right)-\gamma_{\downarrow} c_{x_{j} \downarrow}^{\#_{j}}\left(s_{j}\right) \otimes c_{x_{j} \uparrow}^{\#_{j}}\left(s_{j}\right) \gamma_{\uparrow}\right] \mathrm{e}^{-\beta H_{\mathrm{ext}} \mathscr{R}^{-1}}
\end{aligned}
$$

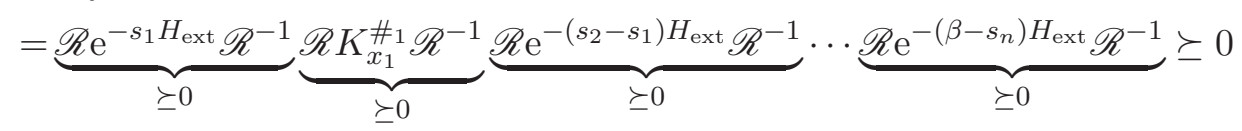

w.r.t. $\mathscr{L}^{2}(\mathfrak{H})_{+}$. Hence, by Theorem $\underline{3.10}$, we obtain the second inequality in $(7.13)$. 


\subsection{Proof of Corollary 7.5}

Let

$$
A_{x,+}=\bar{n}_{x \uparrow} n_{x \downarrow} \otimes \mathbb{1}+\mathbb{1} \otimes \bar{n}_{x \uparrow} n_{x \downarrow}, \quad A_{x,-}=\bar{n}_{x \uparrow} \otimes n_{x \downarrow}+n_{x \downarrow} \otimes \bar{n}_{x \uparrow} .
$$

Observe that

$$
\begin{aligned}
\mathscr{U} A_{x,+} \mathscr{U}^{*} & =\frac{1}{2}\left\{\mathcal{L}\left(\mathcal{N}_{x}\right) \mathcal{R}\left(\mathcal{N}_{x}\right)+\mathcal{L}\left(\mathcal{M}_{x}\right) \mathcal{R}\left(\mathcal{M}_{x}\right)\right\} \\
\mathscr{U} A_{x,-} \mathscr{U}^{*} & =\frac{1}{2}\left\{\mathcal{L}\left(\mathcal{N}_{x}\right) \mathcal{R}\left(\mathcal{N}_{x}\right)-\mathcal{L}\left(\mathcal{M}_{x}\right) \mathcal{R}\left(\mathcal{M}_{x}\right)\right\}
\end{aligned}
$$

Clearly, $\mathcal{L}\left(\mathcal{N}_{x}\right) \mathcal{R}\left(\mathcal{N}_{x}\right) \succeq 0, \quad \mathcal{L}\left(\mathcal{M}_{x}\right) \mathcal{R}\left(\mathcal{M}_{x}\right) \succeq 0$ w.r.t. $\mathscr{L}^{2}(\mathfrak{X})_{+}$. Thus, we have

$$
\mathscr{U}\left(\prod_{j=1}^{n} A_{x_{j},+}-\overrightarrow{\prod_{j=1}^{n}} A_{x_{j},-}\right) \mathscr{U}^{*} \succeq 0 \text { w.r.t. } \mathscr{L}^{2}(\mathfrak{X})_{+} .
$$

By applying Theorem 3.10, we obtain the first inequality in (7.16). Proof of the second inequality in (7.16) is similar to that of Section 7.5.2.

\section{Concluding remarks}

Let $\mathfrak{P}$ be a self-dual cone in the Hilbert space $\mathfrak{H}$. Let $H_{0}$ and $V$ be self-adjoint operators in $\mathfrak{H}$. For simplicity, we assume that $H_{0}$ and $V$ are bounded $17 H_{0}$ is the free Hamiltonian and $V$ is the interaction. The system's Hamiltonian is given by $H=H_{0}-V$. Through our studies of the quantum Griffiths inequality, we recognize that the following are model-independent properties 18

$\left(\mathfrak{P}\right.$ i) $\mathrm{e}^{-\beta H_{0}} \unrhd 0$ w.r.t. $\mathfrak{P}$ for all $\beta \geq 0$.

( $\mathfrak{P}$ ii) $V \unrhd 0$ w.r.t. $\mathfrak{P}$.

( $\mathfrak{P}$ ii) is equivalent to $-V \unlhd 0$ w.r.t. $\mathfrak{P}$. Thus, if ( $\mathfrak{P}$ ii) is satisfied, we say that $-V$ is attractive w.r.t. $\mathfrak{P}$. As we have discussed in the previous sections, when we construct the Griffiths inequality, it is most important to find a self-dual cone $\mathfrak{P}$ such that $-V$ becomes attractive w.r.t. $\mathfrak{P}$. In this step, we are faced with the following difficulty: in general, there are infinitely many self-dual cones in a single Hilbert space. Let us assume that $(\mathfrak{P}$ i) and $(\mathfrak{P}$ ii) are satisfied by choosing some self-dual cone $\mathfrak{P}$. Now let us choose another self-dual cone $\mathfrak{P}^{\prime}$. Even if $(\mathfrak{P}$ i) and ( $\mathfrak{P}$ ii) are satisfied, we can never conclude that $\left(\mathfrak{P}^{\prime}\right.$ i) and ( $\mathfrak{P}^{\prime}$ ii) are fulfilled. Therefore, to apply our theory, we have to choose a proper self-dual cone $\mathfrak{P}$ such that $(\mathfrak{P}$ i) and $(\mathfrak{P}$ ii) are satisfied. In other words, a suitable choice of a self-dual cone makes the interaction $-V$ attractive. In this sense, our theory is a kind of representation theory of attraction.

We remark upon some additional conclusions from ( $\mathfrak{P}$ i) and ( $\mathfrak{P}$ ii). First, we obtain the positivity of a ground state.

\footnotetext{
${ }^{17}$ This assumption can be relaxed [4].

${ }^{18}$ Even when we show the second Griffiths inequality, the properties ( $\mathfrak{P}$ i) and ( $\mathfrak{P}$ ii) are essential for our proof. Namely, $(\mathfrak{P}$ i) and $(\mathfrak{P}$ ii) still hold true for the extended Hamiltonian acting in the doubled Hilbert space $\mathfrak{H} \otimes \mathfrak{H}$, see Sections 20.
} 
Theorem 8.1 44] Assume ( $\mathfrak{P}$ i) and ( $\mathfrak{P}$ ii). Assume that $E=\inf \operatorname{spec}(H)$ is an eigenvalue of $H$. Then there exists a nonzero vector $\psi \in \operatorname{ker}(H-E)$ such that $\psi \geq 0$ w.r.t. $\mathfrak{P}$. Namely, among all the ground states of $H$, there exists at least one ground state that is positive w.r.t. $\mathfrak{P}$.

Theorem 8.2 claims that the attractive interaction makes the system more stable.

Theorem 8.2 [4] Assume $\left(\mathfrak{P}\right.$ i) and $\left(\mathfrak{P}\right.$ ii). Let $E_{0}=\inf \operatorname{spec}\left(H_{0}\right)$. Then $E \leq E_{0}$.

To describe further effects of $(\mathfrak{P}$ i) and ( $\mathfrak{P}$ ii), we define the following:

Definition 8.3 (i) A vector $y \in \mathfrak{H}$ is called strictly positive w.r.t. $\mathfrak{P}$, whenever $\langle x \mid y\rangle>0$ for all $x \in \mathfrak{P} \backslash\{0\}$. We write this as $y>0$ w.r.t. $\mathfrak{P}$.

(ii) We write $A \triangleright 0$ w.r.t. $\mathfrak{P}$, if $A x>0$ w.r.t. $\mathfrak{P}$ for all $x \in \mathfrak{P} \backslash\{0\}$. In this case, we say that $A$ improves the positivity w.r.t. $\mathfrak{P}$. $\diamond$

Theorem 8.4 [12, 43] Assume $\left(\mathfrak{P}\right.$ i) and $\left(\mathfrak{P}\right.$ ii). Assume that $\mathrm{e}^{-\beta H} \triangleright 0$ w.r.t. $\mathfrak{P}$ for all $\beta>0$. If $E=\inf \operatorname{spec}(H)$ is an eigenvalue, then $\operatorname{dim} \operatorname{ker}(H-E)=1$ (equivalently, if $H$ has a ground state, then it is unique). Moreover, the unique ground state is strictly positive w.r.t. $\mathfrak{P}$.

Remark 8.5 If we impose additional conditions on $V$, we can prove $E<E_{0}$ [44]. $\diamond$

As a corollary of Theorem 8.4, we obtain information about structure of the ground state.

Corollary 8.6 Let $G$ be a group and let $\pi$ be an irreducible representation of $G$ on $\mathfrak{H}$. Assume that $\pi_{g} \unrhd 0$ w.r.t. $\mathfrak{P}$ for all $g \in G$. Under the same assumptions as in Theorem 8.4. let $\varphi$ be the ground state of $H$, i.e., $\varphi \in \operatorname{ker}(H-E)$. Then we have $\pi_{g} \varphi=\varphi$ for all $g \in G$.

In the theory of strongly correlated electron systems, we can investigate the magnetic properties of the ground state by Theorem 8.4 and Corollary 8.6 [14, 15, 37, 38, 39, 40, 43, 45, 46, 48, 49, 50, 55, 56, 57]. Furthermore, we can find the same structures in several areas, e.g., in the quantum field theory [16, 22, 26, 43, 46, 47, 51, open quantum systems [41, topological orders [30, 31], and the theory of phase transitions [1, 2, 3, 11, 17, 18, 21, 27, 33. These facts indicate that ( $\mathfrak{P}$ i) and ( $\mathfrak{P}$ ii) are universal expressions of the notion of correlations. If this hypothesis is correct, then several areas could be described by the same language and a new discovery in some areas would automatically influence other areas. To reinforce this vision of unification, we must continue to collect evidence.

\section{A Fundamental properties of operator inequalities asso- ciated with self-dual cones}

\section{A.1 Positivity preserving operators}

In this appendix, we review useful operator inequalities studied in [43].

Let $\mathfrak{H}$ be a complex Hilbert space and $\mathfrak{P}$ be a self-dual cone in $\mathfrak{H}$. 
Proposition A.1 Let $\left\{x_{n}\right\}_{n \in \mathbb{N}}$ be a CONS of $\mathfrak{H}$. Assume that $x_{n} \in \mathfrak{P}$ for all $n \in \mathbb{N}$. Assume that $A \unrhd 0$ w.r.t. $\mathfrak{P}$. Then we have $\operatorname{Tr}[A] \geq 0$.

Proof. Since $x_{n} \in \mathfrak{P}$, we see that $\left\langle x_{n} \mid A x_{n}\right\rangle \geq 0$ for all $n \in \mathbb{N}$. Thus, we arrive at $\operatorname{Tr}[A]=\sum_{n=1}^{\infty}\left\langle x_{n} \mid A x_{n}\right\rangle \geq 0$.

Proposition A.2 Let $N=\operatorname{dim} \mathfrak{H} \in \mathbb{N} \cup\{\infty\}$. Let $\left\{x_{n}\right\}_{n=1}^{N}$ be a CONS of $\mathfrak{H}$. Assume that $x_{n} \in \mathfrak{P}$ for all $n \in\{1, \ldots, N\} 19$ Then the following (i) and (ii) are equivalent.

(i) $A \unrhd 0$ w.r.t. $\mathfrak{P}$.

(ii) $A_{m n}=\left\langle x_{m} \mid A x_{n}\right\rangle \geq 0$ for all $m, n \in\{1, \ldots, N\}$.

Proof. (i) $\Longrightarrow$ (ii): Trivial.

(ii) $\Longrightarrow$ (i): Let $w, z \in \mathfrak{P}$. Then we can write

$$
\begin{aligned}
& w=\sum_{n=1}^{N} c_{n} x_{n}, \quad c_{n}=\left\langle w \mid x_{n}\right\rangle, \\
& z=\sum_{n=1}^{N} d_{n} x_{n}, \quad d_{n}=\left\langle z \mid x_{n}\right\rangle .
\end{aligned}
$$

Since $w, z \geq 0$ w.r.t. $\mathfrak{P}$, we see that $c_{n} \geq 0, d_{n} \geq 0$ for all $n \in \mathbb{N}$. Thus, we have

$$
\langle w \mid A z\rangle=\sum_{m, n=1}^{N} c_{m} d_{n} A_{m n} \geq 0 .
$$

Since $\mathfrak{P}$ is self-dual, we have $A z \geq 0$ w.r.t. $\mathfrak{P}$. Thus, we conclude that $A \unrhd 0$ w.r.t. $\mathfrak{P}$.

Proposition A.3 Assume that $A \unrhd 0$ w.r.t. $\mathfrak{P}$. Then $\mathrm{e}^{\beta A} \unrhd 0$ w.r.t. $\mathfrak{P}$ for all $\beta \geq 0$.

Proof. Since $A \unrhd 0$ w.r.t. $\mathfrak{P}$, it holds that $A^{n} \unrhd 0$ w.r.t. $\mathfrak{P}$ for all $n \in \mathbb{N}$. Thus,

$$
\mathrm{e}^{\beta A}=\sum_{n \geq 0} \underbrace{\frac{\beta^{n}}{n !}}_{\geq 0} \underbrace{A^{n}}_{\unrhd 0} \unrhd 0 \quad \text { w.r.t. } \mathfrak{P} \text { for all } \beta \geq 0 .
$$

Proposition A.4 Assume that $\mathrm{e}^{\beta A} \unrhd 0$ and $\mathrm{e}^{\beta B} \unrhd 0$ w.r.t. $\mathfrak{P}$ for all $\beta \geq 0$. Then $\mathrm{e}^{\beta(A+B)} \unrhd 0$ w.r.t. $\mathfrak{P}$ for all $\beta \geq 0$.

Proof. Note that $\mathrm{e}^{\beta A} \mathrm{e}^{\beta B} \unrhd 0$ w.r.t. $\mathfrak{P}$ for all $\beta \geq 0$. Thus, $\left(\mathrm{e}^{\beta A / n} \mathrm{e}^{\beta B / n}\right)^{n} \unrhd 0$ w.r.t. $\mathfrak{P}$ for all $\beta \geq 0$ and $n \in \mathbb{N}$. By the Trotter-Kato product formula, we obtain the desired assertion.

The following proposition is repeatedly used in this study.

Proposition A.5 Assume the following:

\footnotetext{
${ }^{19}$ In the case where $N=\infty$, the symbol $\{1, \ldots, N\}$ denotes $\mathbb{N}$.
} 
(i) $\mathrm{e}^{\beta A} \unrhd 0$ w.r.t. $\mathfrak{P}$ for all $\beta \geq 0$.

(ii) $B \unrhd 0$ w.r.t. $\mathfrak{P}$.

Then we have $\mathrm{e}^{\beta(A+B)} \unrhd 0$ w.r.t. $\mathfrak{P}$ for all $\beta \geq 0$.

Proof. By (ii) and Proposition $A .3$, it holds that $\mathrm{e}^{\beta B} \unrhd 0$ w.r.t. $\mathfrak{P}$ for all $\beta \geq 0$. Thus, applying Proposition A.4, we conclude the assertion.

Proposition A.6 Let $A$ be a positive self-adjoint operator. Assume that $\mathrm{e}^{-\beta A} \unrhd 0$ w.r.t. $\mathfrak{P}$ for all $\beta \geq 0$. Assume that $E=\inf \operatorname{spec}(A)$ is an eigenvalue of $A$. Then there exists a nonzero vector $x \in \operatorname{ker}(A-E)$ such that $x \geq 0$ w.r.t. $\mathfrak{P}$.

Proof. STEP 1. Let $J$ be an antilinear involution given by Proposition A.7 below. Set $\mathfrak{H}_{J}=\{x \in \mathfrak{H} \mid J x=x\}$. We will show that $\operatorname{ker}(A-E) \cap \mathfrak{H}_{J} \neq\{0\}$.

To see this, let $x \in \operatorname{ker}(A-E)$. Then we have the decomposition $x=\Re x+i \Im x$ with $\Re x=\frac{1}{2}(\mathbb{1}+J) x$ and $\Im x=\frac{1}{2 i}(\mathbb{1}-J) x$. Clearly , $\Re x, \Im x \in \mathfrak{H}_{J}$. Since $x \neq 0$, it holds that $\Re x \neq 0$ or $\Im x \neq 0$. Since $\mathrm{e}^{-\beta A} \unrhd 0$ w.r.t. $\mathfrak{P}$ for all $\beta \geq 0, A$ commutes with $J$. Thus, $\Re x, \Im x \in \operatorname{ker}(A-E) \cap \mathfrak{H}_{J}$.

STEP 2. Take $x \in \operatorname{ker}(A-E) \cap \mathfrak{H}_{J}$. By Proposition A.7 (iii), we have a unique decomposition $x=x_{+}-x_{-}$, where $x_{ \pm} \in \mathfrak{P}$ and $\left\langle x_{+} \mid x_{-}\right\rangle=0$. Let $|x|=x_{+}+x_{-}$. Then we have

$$
\mathrm{e}^{-\beta E}\|x\|=\left\langle x \mid \mathrm{e}^{-\beta A} x\right\rangle \leq\left\langle|x|\left|\mathrm{e}^{-\beta A}\right| x \mid\right\rangle \leq \mathrm{e}^{-\beta E} \underbrace{\||x|\|}_{=\|x\|} .
$$

Thus, $|x| \in \operatorname{ker}(A-E)$. Clearly, $|x| \geq 0$ w.r.t. $\mathfrak{P}$.

Proposition A.7 A self-dual cone $\mathfrak{P}$ has the following properties:

(i) $\mathfrak{P} \cap(-\mathfrak{P})=\{0\}$.

(ii) There exists a unique antilinear involution $J$ in $\mathfrak{H}$ such that $J x=x$ for all $x \in \mathfrak{P}$.

(iii) Each element $x \in \mathfrak{H}$ with $J x=x$ has a unique decomposition $x=x_{+}-x_{-}$where $x_{+}, x_{-} \in \mathfrak{P}$ and $\left\langle x_{+} \mid x_{-}\right\rangle=0$.

(iv) $\mathfrak{H}$ is linearly spanned by $\mathfrak{P}$.

Proof. See, e.g., [5].

\section{A.2 Reflection positive operators}

To apply Theorem 3.11, it is crucial to show that $\mathrm{e}^{-\beta H} \succeq 0$ w.r.t. $\mathscr{L}^{2}(\mathfrak{H})+$ for all $\beta>0$. The following proposition is often useful in proving this condition:

Proposition A.8 Let $H_{0}$ be a self-adjoint operator on $\mathscr{L}^{2}(\mathfrak{H})$ bounded from below. Let $V \in \mathscr{B}\left(\mathscr{L}^{2}(\mathfrak{H})\right)$ be self-adjoint. Assume the following:

(i) $\mathrm{e}^{-\beta H_{0}} \succeq 0$ w.r.t. $\mathscr{L}^{2}(\mathfrak{H})_{+}$for all $\beta \geq 0$.

(ii) $V \succeq 0$ w.r.t. $\mathscr{L}^{2}(\mathfrak{H})_{+}$. 
Let $H=H_{0}-V$. We have $\mathrm{e}^{-\beta H} \succeq 0$ w.r.t. $\mathscr{L}^{2}(\mathfrak{H})_{+}$for all $\beta \geq 0$.

Proof. Note that

$$
\mathrm{e}^{\beta V}=\sum_{n \geq 0} \underbrace{\frac{\beta^{n}}{n !}}_{\geq 0} \underbrace{V^{n}}_{\succeq 0} \succeq 0 \quad \text { w.r.t. } \mathscr{L}^{2}(\mathfrak{H})_{+} .
$$

Thus, by the Trotter-Kato product formula, we obtain

$$
\mathrm{e}^{-\beta H}=\mathrm{s}-\lim _{n \rightarrow \infty}(\underbrace{\mathrm{e}^{-\beta H_{0} / n}}_{\succeq 0} \underbrace{e^{\beta V / n}}_{\succeq 0})^{n} \succeq 0 \quad \text { w.r.t. } \mathscr{L}^{2}(\mathfrak{H})_{+} \text {for all } \beta \geq 0,
$$

where s- $\lim _{n \rightarrow \infty}$ means the strong limit.

Corollary A.9 Let $H_{0}=\mathcal{L}(A)+\mathcal{R}(A)$, where $A$ is self-adjoint and bounded from below. Let

$$
V=\sum_{j=1}^{\infty} \mathcal{L}\left(B_{j}\right) \mathcal{R}\left(B_{j}\right)
$$

where $B_{j} \in \mathscr{B}(\mathfrak{H})$ is self-adjoint and the right hand side of (A.8) is a weak convergent sum. Define $H=H_{0}-V$. Then we obtain $\mathrm{e}^{-\beta H} \succeq 0$ w.r.t. $\mathscr{L}^{2}(\mathfrak{H})_{+}$for all $\beta \geq 0$.

Proof. Observe that $\mathrm{e}^{-\beta H_{0}}=\mathcal{L}\left(\mathrm{e}^{-\beta A}\right) \mathcal{R}\left(\mathrm{e}^{-\beta A}\right) \succeq 0$ w.r.t. $\mathscr{L}^{2}(\mathfrak{H})_{+}$for all $\beta \geq 0$. Since $V \succeq 0$ w.r.t. $\mathscr{L}^{2}(\mathfrak{H})_{+}$, we obtain the desired assertion by Proposition A.8.

The following lemma will be often useful:

Lemma A.10 Let $A_{j}, j=1, \ldots, N$ be a bounded operator acting in $\mathfrak{H}$. Let $M=\left(M_{i j}\right)$ be a positive semidefinite $N \times N$ matrix. Then we have

$$
\sum_{i, j=1}^{N} M_{i j} \mathcal{L}\left(A_{i}^{*}\right) \mathcal{R}\left(A_{j}\right) \succeq 0 \text { w.r.t. } \mathscr{L}^{2}(\mathfrak{H})_{+} .
$$

Proof. There exists a unitary matrix $U$ such that $M=U^{*} D U$, where $D=\operatorname{diag}\left(\lambda_{j}\right)$ is a diagonal matrix with $\lambda_{j} \geq 0$. Set $\tilde{A}_{i}=\sum_{j=1}^{N} U_{i j} A_{j}$. Then we see

$$
\text { LHS of }(\underline{\mathrm{A} .9})=\sum_{j=1}^{N} \lambda_{j} \mathcal{L}\left(\tilde{A}_{j}^{*}\right) \mathcal{R}\left(\tilde{A}_{j}\right) \succeq 0 \quad \text { w.r.t. } \mathscr{L}^{2}(\mathfrak{H})_{+} \cdot
$$

This completes the proof.

\section{References}

[1] M. Aizenman, E. H. Lieb, R. Seiringer, J. P. Solovej, J. Yngvason, Bose-Einstein quantum phase transition in an optical lattice model, Phys. Rev. A 70 (2004), 023612 . 
[2] C. Albert, L. Ferrari, J. Fröhlich, B. Schlein, Magnetism and the Weiss exchange field-A theoretical analysis motivated by recent experiments, Jour. Stat. Phys. 125 (2006), 77-124.

[3] M. Biskup, Reflection positivity and phase transitions in lattice spin models, Methods of contemporary mathematical statistical physics, 1-86, Lecture Notes in Math., 1970, Springer, Berlin, 2009.

[4] J. E. Bjornberg, G. R. Grimmett, The phase transition of the quantum Ising model is sharp, Jour. Stat. Phys. 136 (2009), 231-273.

[5] W. Bös, Direct integrals of selfdual cones and standard forms of von Neumann algebras, Invent. Math. 37 (1976), 241-251.

[6] J. Bricmont, J.-R. Fontaine, J. L. Lebowitz, E. H. Lieb, T. Spencer, Lattice systems with a continuous symmetry. III. Low temperature asymptotic expansion for the plane rotator model, Comm. Math. Phys. 78 (1980), 545-566.

[7] O. Bratteli, A. Kishimoto, D. W. Robinson, Positivity and monotonicity properties of $C_{0}$-semigroups. I, Comm. Math. Phys. 75 (1980), 67-84.

[8] P. Contucci, J. L. Lebowitz, Correlation inequalities for quantum spin systems with quenched centered disorder, Jour. Math. Phys. 37 (2010), 5458-5475.

[9] N. Crawford, D. Ioffe, Random current representation for transverse field Ising model, Comm. Math. Phys. 296 (2010), 447-474.

[10] W. Driessler, L. Landau, J. F. Perez, Estimates of critical lengths and critical temperatures for classical and quantum lattice systems, Jour. Stat. Phys. 20 (1979), 123-162.

[11] F. J. Dyson, E. H. Lieb, B. Simon, Phase transitions in quantum spin systems with isotropic and nonisotropic interactions, Jour. Stat. Phys. 18 (1978), 335-383.

[12] W. G. Faris, Invariant cones and uniqueness of the ground state for fermion systems, Jour. Math. Phys. 13 (1972), 1285-1290.

[13] C. M. Fortuin, P. W. Kasteleyn, J. Ginibre, Correlation inequalities on some partially ordered sets, Comm. Math. Phys. 22 (1971), 89-103.

[14] R. L. Frank, E. H. Lieb, Possible lattice distortions in the Hubbard model for graphene, Phys. Rev. Lett. 107 (2011), 066801.

[15] J. K. Freericks, E. H. Lieb, Ground state of a general electron-phonon Hamiltonian is a spin singlet, Phys. Rev. B 51 (1995), 2812-2821.

[16] J. Fröhlich, On the infrared problem in a model of scalar electrons and massless, scalar bosons, Ann. Inst. H. Poincaré Sect. A (N.S.) 19 (1973), 1-103.

[17] J. Fröhlich, R. Israel, E. H. Lieb, B. Simon, Phase transitions and reflection positivity. I. General theory and long range lattice models, Comm. Math. Phys. 62 (1978), 1-34. 
[18] J. Fröhlich, B. Simon, T. Spencer, Infrared bounds, phase transitions and continuous symmetry breaking, Comm. Math. Phys. 50 (1976), 79-95.

[19] G. Gallavotti, A proof of the Griffiths inequalities for the $X Y$ model, Studies in Appl. Math., L (1971), 89-92.

[20] J. Ginibre, General formulation of Griffiths' inequalities, Comm. Math. Phys. 16 (1970), 310-328.

[21] J. Glimm, A. Jaffe, T. Spencer, Phase transitions for $\varphi_{2}^{4}$ quantum fields, Comm. Math. Phys. 45 (1975), 203-216.

[22] J. Glimm, A. Jaffe, Quantum physics. A functional integral point of view. Second edition. Springer-Verlag, New York, 1987.

[23] R. B. Griffiths, Correlations in Ising ferromagnets. I, Jour. Math. Phys. 8 (1967), 484-488.

[24] R. B. Griffiths, Correlations in Ising ferromagnets. II, Jour. Math. Phys. 8 (1967), 478-483.

[25] R. B. Griffiths, Rigorous results for Ising ferromagnets of arbitrary spin, Jour. Math. Phys. 10 (1969), 1559-1565.

[26] L. Gross, Existence and uniqueness of physical ground states, Jour. Funct. Anal. (1972), 52-109.

[27] O. J. Heilmann, E. H. Lieb, Lattice models for liquid crystals, Jour. Stat. Phys. 20 (1979), 679-693.

[28] C. A. Hurst, S. Sherman, Griffiths' theorems for the ferromagnetic Heisenberg model, Phys. Rev. Lett. 22 (1969), 1357-1358.

[29] W. Lenz, Beitrag zum verständnis der magnetischen Erscheinungen in festen körpern, Phys. Zeitschr. 21 (1920), 613-615.

[30] A. Jaffe, F. L. Pedrocchi, Reflection positivity for Majoranas, Ann. Henri Poincaré 16 (2015), 189-203.

[31] A. Jaffe, F. L. Pedrocchi, Reflection positivity for parafermions, Comm. Math. Phys. 337 (2015), 455-472.

[32] D. G. Kelly, S. Sherman, General Griffiths' inequalities on correlations in Ising ferromagnets, Jour. Math. Phys. 9 (1968), 466-484.

[33] T. Kennedy, E. H. Lieb, B. S. Shastry, Existence of Neel order in some spin-1/2 Heisenberg antiferromagnets, Jour. Stat. Phys. 53 (1988), 1019-1030.

[34] J. R. Kirkwood, L. E. Thomas, Expansions and phase transitions for the ground state of quantum Ising lattice systems, Comm. Math. Phys. 88 (1983), 569-580.

[35] A. Kishimoto, D. W. Robinson, Positivity and monotonicity properties of $C_{0}$ -semigroups. II, Comm. Math. Phys. 75 (1980), 85-101. 
[36] A. Klein, J. F. Perez, Localization in the ground state of a disordered array of quantum rotators. Comm. Math. Phys. 147 (1992), 241-252.

[37] E. H. Lieb, D. Mattis, Ordering energy levels of interacting spin systems, Jour. Math. Phys. 3 (1962), 749-751.

[38] E. H. Lieb, Two theorems on the Hubbard model, Phys. Rev. Lett. 62 (1989), 1201-1204.

[39] E. H. Lieb, B. Nachtergaele, Stability of the Peierls instability for ring-shaped molecules, Phys. Rev. B 51 (1995), 4777-4791.

[40] E. H. Lieb, P. Schupp, Ground state properties of a fully frustrated quantum spin system, Phys. Rev. Lett. 83 (1999), 5362-5365.

[41] G. Lindbald, On the generators of quantum dynamical semigroups, Comm. Math. Phys. 48 (1976), 119-130.

[42] Y. Miura, On order of operators preserving selfdual cones in standard forms, Far East J. Math. Sci. (FJMS) 8 (2003), 1-9.

[43] T. Miyao, Nondegeneracy of ground states in nonrelativistic quantum field theory, Jour. Operator Theory 64 (2010), 207-241.

[44] T. Miyao, Self-dual cone analysis in condensed matter physics, Rev. Math. Phys. 23 (2011), 749-822.

[45] T. Miyao, Ground state properties of the SSH model, Jour. Stat. Phys. 149 (2012), 519-550.

[46] T. Miyao, Monotonicity of the polaron energy II: General theory of operator monotonicity, Jour. Stat. Phys. 153 (2013), 70-92.

[47] T. Miyao, Monotonicity of the polaron energy, Rep. Math. Phys. 74 (2014), 379398.

[48] T. Miyao, Rigorous results concerning the Holstein-Hubbard model, to appear in Ann. Henri Poicare, arXiv:1402.5202,

[49] T. Miyao, Upper bounds on the charge susceptibility of many-electron systems coupled to the quantized radiation field, Lett. Math. Phys. 105 (2015), 1119-1133.

[50] Y. Nagaoka, Ferromagnetism in a narrow, almost half-filled $s$ band, Phys. Rev. 147 (1966), 392-405.

[51] K. Osterwalder, R. Schrader, Axioms for Euclidean Green's functions, Comm. Math. Phys. (1973), 83-112. Axioms for Euclidean Green's functions. II. With an appendix by Stephen Summers, Comm. Math. Phys. 42 (1975), 281-305.

[52] J. K. Percus, Correlation inequalities for Ising spin lattices. Comm. Math. Phys. 40 (1975), 283-308. 
[53] M. Reed, B. Simon, Methods of Modern Mathematical Physics Vol. IV, Academic Press, New York, 1978.

[54] S. Sachdev, Quantum Phase Transitions, Cambridge University Press. (2nd ed.), 2011.

[55] S. H. Shen, Strongly correlated electron systems: Spin-reflection positivity and some rigorous results, Int. J. Mod. Phys. B 12 (1998), 709.

[56] M. Sigrist, H. Tsunetsugu, K. Ueda, Rigorous results for the one-electron Kondolattice model, Phys. Rev. Lett. 67 (1991), 2211-2214.

[57] G-S. Tian, Lieb's spin-reflection positivity methods and its applications to strongly correlated electron systems, Jour. Stat. Phys. 116 (2004), 629-680. 